\title{
Neue Einblicke in das Gravettien von Willendorf II. Die Steinartefakte der Grabung 1993
}

\author{
Viola C. Schmid \\ Marjolein D. Bosch \\ Michael Brandl \\ Michael Götzinger \\ Philip R. Nigst
}

\section{Zusammenfassung}

Die gut datierte stratigrafische Abfolge von Willendorf II ist eine Referenzfundstelle für das Jungpaläolithikum im Allgemeinen und das Gravettien im Speziellen. Im Jahr 1993 führte ein gemeinsames Team des Institut Royal des Sciences Naturelles de Belgique (Brüssel, Belgien) und der Universität Wien eine Neuuntersuchung an der seit Ende des 19. Jhs. bekannten Löss-Fundstelle im Donautal durch. Bei diesen Grabungsaktivitäten wurden 391 Fundstücke geborgen, die vorwiegend aus den archäologischen Horizonten (AH) 6 und 8 stammen. Im Zuge der hier vorliegenden Arbeit wurde eine technologische Analyse der Steinartefakte basierend auf einem merkmalanalytischen Aufnahmesystem durchgeführt. Diese Studie ergab, dass die Inventare der beiden $\mathrm{AH}$ sich in Bezug auf Rohmaterialnutzung und Werkzeugbestand markant voneinander unterscheiden.

AH 6 weist einen hohen Anteil an nicht-lokalem baltischem Feuerstein auf. Die unidirektional ausgerichtete Abbaustrategie zielte auf die Produktion von Lamellen besonders an hochwertigen Rohmaterialien ab, um diese zu Rückenelementen, die als Projektile Verwendung fanden, weiterzuverarbeiten. Neben der unidirektionalen, volumetrischen Lamellenabbaustrategie wurden auch Stichel als Lamellenkerne genutzt. Das Werkzeugspektrum enthält neben den Rückenelementen Stichel, Kratzer und Kantenretuschen. In AH 8 dominieren lokale Rohmaterialien wie Quarzit und Kieselkalk. Die unidirektional ausgerichtete Abbaustrategie zielte auf die Herstellung von Klingen ab. Der Werkzeugbestand umfasst eine Bandbreite an verschiedenen Werkzeugtypen. Am häufigsten treten Ausgesplitterte Stücke auf, des Weiteren wurden Spitzklingen, Stichel, Kratzer, Kantenretuschen, Endretuschen, ein Bohrer mit Kantenretusche und ein gezähntes Stück festgestellt.

Die Unterschiede in AH 6 und AH 8 sowie die Ähnlichkeiten von $\mathrm{AH} 6$ zu AH 5 und AH 6 der Altgrabungen signalisieren, dass AH 6 und AH 8 der Grabung 1993 nicht derselben Phase des Gravettien angehören. Die Jäger und Sammler zur Zeit von AH 6 weisen ein technologisches, technoökonomisches und soziotechnologisches Verhalten auf, das eher einem frühen Gravettien entsprechen und den Innovationen des Pavlovien vorausgehen dürfte, während der AH 8 den Forschungskonsens bestätigend mit den Charakteristika des Pavlovien übereinstimmt.

\section{Schlüsselbegriffe}

Gravettien, Pavlovien, chrono-kulturelle Abfolge, Steintechnologie, Willendorf II.

\begin{abstract}
New Insights into the Gravettian of Willendorf II. The Lithic Artefacts of the 1993 Excavation

The well-dated stratigraphic sequence of Willendorf II is a reference site for the Upper Palaeolithic in general and the Gravettian in particular. In 1993, a joint team from the Institut Royal des Sciences Naturelles de Belgique (Brussels, Belgium) and the University of Vienna carried out excavations at this loess site in the Danube Valley known since the end of the $19^{\text {th }}$ century. During the excavation season, the team recovered 391 artefacts, the majority attributed to AH 6 and 8 . We performed a technological study based on an attribute analysis of the lithic artefacts. This study demonstrates that the assemblages of AH 6 and 8 differ significantly in terms of technology and techno-economy.
\end{abstract}

AH 6 has a high proportion of non-local erratic flint. The unidirectional reduction strategy aimed at the production of bladelets, especially on high-quality raw materials, to further modify them into backed elements that could be used as projectiles. In addition to the unidirectional, volumetric reduction strategy, burins were also exploited as bladelet cores. Apart from the manufacture of backed elements, the tool spectrum contains burins, end scrapers and pieces with edge retouch. AH 8 is dominated by local raw materials, such as quartzite and siliceous limestone. The unidirectional reduction strategy aimed at the production of blades. The toolkit includes a range of different tool types. Splintered pieces occur most frequently. Furthermore, pointed blades, burins, end scrapers, edge retouches, truncations, a borer with edge retouch, and a notched piece are among the tools.

The differences between AH 6 and AH 8 and the similarities of $\mathrm{AH} 6$ to $\mathrm{AH} 5$ and $\mathrm{AH} 6$ of the old excavations highlight that the AH 6 and AH 8 of the 1993 excavations do not belong to the same phase of the Gravettian technocomplex. The hunter-gatherers of AH 6 show technological, techno-economic and socio-technological behaviours more likely to correspond to the early Gravettian, while AH 8 shows diagnostic features of the Pavlovian, thereby confirming 
the dominant view among researchers that AH 8 of Willendorf II belongs to the Pavlovian.

\section{Keywords}

Gravettian, Pavlovian, chrono-cultural sequence, lithic technology, Willendorf II.

\section{Einleitung}

Willendorf II stellt mit seiner Chronostratigrafie neben Fundstellen wie Mitoc-Malu Galben (Rumänien), ${ }^{1}$ Abri Pataud (Frankreich) ${ }^{2}$ und Molodova V (Ukraine) ${ }^{3}$ eine der Referenz-Stratigrafien des älteren und mittleren Jungpaläolithikums Europas dar. ${ }^{4}$ Vor allem aber beruht auch die Gliederung des mitteleuropäischen Gravettien zu großen Teilen auf der chrono-kulturellen Sequenz von Willendorf II. ${ }^{5}$

In Mitteleuropa etablierte sich der Begriff des Gravettien als Abgrenzung zum Aurignacien im Gegensatz zu Frankreich, wo eine terminologische Auseinandersetzung bereits in den 1930er Jahren ausgelöst wurde, ${ }^{6}$ erst wesentlich später. Zunächst wurde dieser jungpaläolithische Technokomplex als Jung- bzw. Spätaurignacien angesprochen. In den 1920er Jahren zählte Willendorf II zur damals von Josef Bayer neu eingeführten Kulturstufe des „Aggsbachien“, welche er nach dem Fundort Aggsbach an der Donau (Niederösterreich) benannte. ' Diese Kulturgruppe entstammte laut Bayer einer Kielkratzer-freien Tradition aus dem Osten und erreichte in der sogenannten zweiten Schmalklingenwelle Mitteleuropa, wo sie sich auf das Kielkratzer-führende Aurignacien niederschlug. ${ }^{8}$ Willendorf II als gut stratifizierte Freilandfundstelle, bei welcher sich der Kielkratzer-führende archäologische Horizont $(\mathrm{AH})^{9}$ 4 klar von den darüber nachfolgenden Kielkratzer-freien AH 5 bis 9 unterschied, muss für Bayer eine wesentliche Rolle bei der Definition des „Aggsbachien“ gespielt haben. ${ }^{10}$ Der Terminus „Aggsbachien“ konnte sich jedoch

\footnotetext{
1 OtTe, Chirica, Haesaerts 2007.

2 Movius 1977. - Higham et al. 2011.

3 Chernysh 1961. - OTt'e et al. 1996. - Haesaerts et al. 2003. NoIRET 2004.

4 Haesaerts et al. 1996. - Nigst et al. 2014.

5 Otтe 1991. - Svoboda 1994a. - Haesaerts et al. 1996. - OtTe et

al. 1996. - Moreau 2009.

6 Siehe Peyrony 1933. - Peyrony 1937. - Peyrony 1946.

7 BAYER 1928. - BAYER 1929.

8 Felgenhauer 1995-1996, 252. - Moreau 2009, 34.

9 In älterer Literatur als Kulturschicht angesprochen, hier aber als $\mathrm{AH}$, wie auch in den neueren Grabungen von Nigst, Trnka und Viola.

10 Moreau 2009, 35.
}

nicht halten und der übergeordnete, überregionale Begriff „Ostgravettien“ setzte sich durch, da eine Bestrebung nach der Heraushebung der verbindenden anstelle der trennenden Elemente vorherrschte. ${ }^{11}$

Bohuslav Klíma führte dann basierend auf seinen Grabungen in Pavlov I (Südmähren) den Begriff Pavlovien als regionale Ausprägung des Gravettien in der Mitteldonaugegend ein. ${ }^{2}$ Obwohl der Terminus Pavlovien ob seiner unklaren geografischen Abgrenzung kritisiert wurde, ${ }^{13}$ wird er häufig benutzt und gilt einigen Forschern als die ältere Stufe (30.000-25.000 BP, 35.500-29.000 cal BP $\left.{ }^{14}\right)$ des Gravettien, vorwiegend dokumentiert im niederösterreichisch-mährisch-südpolnischen Donaukorridor. ${ }^{15}$ Nach dieser Ansicht wurden neben Willendorf II pavlovienzeitliche Besiedlungen unter anderem in Krems-Wachtberg, ${ }^{16}$ Krems-Hundssteig ${ }^{17}$ (beide in Niederösterreich), Dolní Věstonice I-II, ${ }^{18}$ Pavlov I, ${ }^{19}$ Predmostí, ${ }^{20}$ Jarošov IIPodvršta, ${ }^{21}$ Milovice IV (alle in Mähren), ${ }^{22}$ Henryków 15 (Südpolen) ${ }^{23}$ und vielleicht auch in Grub/Kranawetberg - AH 4 (Niederösterreich) ${ }^{24}$ nachgewiesen. Nach Jiří Svoboda geht dem entwickelten Pavlovien, ${ }^{25}$ welches in einem Zeitraum von 27.000 bis 25.000 BP (31.000-29.000 cal BP) auftritt und AH 6 bis 8 von Willendorf II umfasst, das frühe Pavlovien voraus, welches zwischen 30.000 und 27.000 BP (35.500 und $31.000 \mathrm{cal} \mathrm{BP)} \mathrm{datiert} \mathrm{und} \mathrm{nach} \mathrm{Svoboda}$ den AH 5 von Willendorf II einschließt. Luc Moreau plädiert jedoch anhand von Vergleichsstudien der Steinartefaktinventare von Willendorf II - AH 5, Geißenklösterle AH I (Deutschland) sowie Weinberghöhlen (Deutschland) für eine Abschaffung des Terminus „frühes Pavlovien“, da dieser Begriff den stark einheitlichen kulturellen Charakter der frühen Gravettien-Steinindustrien in der oberen und mittleren Donauregion verschleiert. ${ }^{26}$ Die Gliederung des

\footnotetext{
11 Felgenhauer 1951

12 Klíma 1959.

13 Felgenhauer 1995-1996.

14 Kalibrierte Kalenderjahre nach Nigst et al. 2014. Für Kalibrationskurve, verwendete Kalibrationssoftware und beim Kalibrieren verwendete Parameter siehe NigST et al. 2014.

15 Svoboda 2007.

16 EINWÖGERER 2010.

17 Neugebauer-Maresch 2008.

18 Svoboda 1994a.

19 Svoboda 1994a.

20 Svoboda 1994a.

21 Svoboda 2003.

22 Svoвoda 2010. - Svoboda et al. 2011

23 WILCZYŃSKI et al. 2015. - WIŚNIEWSKI et al. 2015.

24 AntL-Weiser et al. 2010. - ANTL-Weiser 2013.

25 Svoboda 2003, 214.

26 Moreau 2009, 46. - Moreau 2010, 90-91.
} 
Pavlovien beruht ursprünglich weitgehend auf der gut dokumentierten langen Schichtenabfolge von Willendorf II. ${ }^{27}$ Diese Einteilung unterscheidet sich von der Klassifizierung des mitteleuropäischen Gravettien nach Marcel Otte, ${ }^{28}$ die ebenfalls anhand der Chronostratigrafie von Willendorf II erstellt wurde, durch den geografisch enger gefassten Raum sowie eine andere chrono-kulturelle Unterteilung der älteren gravettienzeitlichen Phasen von Willendorf II. ${ }^{29}$

Die Fundstellen des Pavlovien weisen Gemeinsamkeiten in Bezug auf ihre Besiedlungsstrukturen, Mobilitätsstrategien, Subsistenzverhalten, Knochenindustrie, rituelle sowie symbolische Ausprägungen, Kunstherstellung, Rohmaterialökonomie und Steintechnologie auf. ${ }^{30}$ Große, gut organisierte, längerfristig genutzte Freilandfundstellen in erhöhten Lagen von ca. 200 bis $300 \mathrm{~m}$ und entlang von Flüssen sind typisch. Die Siedlungsbereiche zeugen von räumlicher Strukturierung und häufig genutzten Konstruktionen, wie eingetieften Feuerstellen, teilweise mit Steinoder Knochenumstellungen sowie umgebenden Gruben. ${ }^{31}$ Rohmaterialien werden zu großen Anteilen aus weiten Distanzen importiert. ${ }^{32}$ Große Mammutknochenanhäufungen belegen eine intensive Nutzung dieses Großsäugers als Fleisch- sowie Fettversorgungsmittel und als Knochenrohstoffressource. ${ }^{33}$ Die Menschen des Pavlovien dürften unterschiedliche Beschaffungsstrategien angewandt haben, von Knochenaufsammeln über das Essen von Aas bis hin zu gelegentlichen gezielten Jagden. ${ }^{34}$ Das Vorkommen einer Vielzahl von kleineren Tieren wie Feldhasen zeigt außerdem, dass verstärkt auch kleinere, aber vorhersehbarere Nahrungsressourcen genutzt wurden. ${ }^{35}$ In Dolní Věstonice II wurden Überreste von verkohltem Pflanzengewebe gefunden, die auf den Verzehr von pflanzlicher Nahrung hindeuten. ${ }^{36}$ Außerdem gelten die Abdrücke auf gebrannten Lehmbrocken als Belege für Textilien und Pflanzenfasern, die darauf hinweisen, dass Aktivitäten wie Korbmacherei,

27 Svoboda 1994b.

28 ОT'TE 1991.

29 Moreau 2009, 38.

30 Svoboda 2007.

31 Svoboda, Ložek, Vlčen 1996. - Svoboda 2007. - Ziehaus 2007.

- ANTL-Weiser et al. 2010.

32 Svoboda, Ložek, Vlček 1996. - Svoboda 2007. - Ziehaus 2007.

- ANTL-WEISER et al. 2010.

33 Svoboda, Ložek, VlČen 1996. - Svoboda et al. 2000. - Fladerer 2001. - Svoboda 2007. - AntL-Weiser et al. 2010. - Bosch et al. 2012. - Fladerer, SAlcher-Jedrasiak, Händel 2014.

34 Fladerer 2001. - Bosch 2012. - Bosch et al. 2012. - Fladerer, SALCHER-Jedrasiak, HäNdel 2014.

35 Svoboda et al. 2000. - Svoboda 2007.

36 Mason, Hather, Hillman 1994. - Pryor et al. 2013.
Netzweben und Fallenbau ausgeführt wurden. ${ }^{37}$ Die reiche Industrie an organischen Materialien umschließt verschiedenste Formen und Typen von Knochen-, Geweih- und Elfenbeinartefakten. Ahlen, Glätter und Nadeln wurden hergestellt und schaufelartige Objekte treten auf. ${ }^{38}$ Spitzen aus Elfenbein, häufig mit rundem Querschnitt und konischer Basis, könnten als Jagdwaffen gedient haben. ${ }^{39}$ Die Schmuckproduktion ist ein weiteres hervorzuhebendes Charakteristikum. ${ }^{40}$ Ovale und elliptische flache Plättchen zumeist aus Elfenbein, die Durchlochungen an beiden Enden aufweisen und eingeritzte Ornamente tragen, werden als Haarspangen interpretiert. ${ }^{41}$ Des Weiteren zählen Elfenbeinperlen und -anhänger sowie durchlochte Schneckengehäuse und Tierzähne zu den Schmuckgegenständen. ${ }^{42} \mathrm{Ne}-$ ben geometrischen Mustern existieren auch zoomorphe und anthropomorphe Elfenbeinplastiken in Schnitztechnik. ${ }^{43}$ Besonders markant für das Pavlovien sind allerdings modellierte und gebrannte Lehmplastiken. ${ }^{44}$ Die Auffindung von Bestattungen und die Art der Bestattungen lassen auf rituelle Aktivitäten rückschließen. Oft waren die Skelette mit Mammutschulterblättern oder Steinen bedeckt und mit rotem Farbstoff im Kopf- oder Beckenbereich umgeben. ${ }^{45}$

Bezogen auf die typologische Zusammensetzung der Steinwerkzeuge zeichnet sich das Pavlovien durch ein häufigeres Vorkommen von geometrischen Mikrolithen, hauptsächlich Kreissegmenten, Trapezen und Dreiecken, sowie das Auftreten von Gravette- und Mikrogravettespitzen wie auch fléchettes aus. ${ }^{46}$ Außerdem stellen kantenretuschierte Spitzklingen sowie laut Karel Valoch ${ }^{47}$ und Klíma $^{48}$ auch Kostenki-Enden typische Elemente dar. Die Pavlovien-Inventare umfassen zusätzlich einen hohen Anteil an Sticheln. Bohrer, Ausgesplitterte Stücke und Kratzer

37 Soffer et al. 1998.

38 Svoboda et al. 2000. - Svoboda 2007.

39 Klíma 1997. - Zelinkova 2007.

40 Antl-Weiser 1999. - AntL-Weiser 2005. - AntL-Weiser, BosCH 2015.

41 Svoboda et al. 2000.

42 Svoboda et al. 2000. - Ziehaus 2007. - HÄNdel et al. 2009. ANTL-Weiser et al. 2010.

43 Svoboda, Ložek, Vlček 1996. - Svoboda et al. 2000. Verpoorte 2001. - FARbSTEIn 2011a. - FARbSTEIn 2011b.

44 Svoboda, Ložek, Vlček 1996. - Einwögerer 2000. - SvobodA et al. 2000. - Verpoorte 2001. - Svoboda 2007. - Farbstein, Davies 2017.

45 KLíma 1995. - Svoboda, LožEK, VlčeK 1996. - Einwögerer et al. 2006. - Svoboda 2007. - EINWÖgERER 2010. - PetTitT' 2011.

46 Moreau 2009, 43. - Svoboda 2012.

47 VALOCH 1981.

48 Klíma 1968. 
kommen in unterschiedlichen Häufigkeiten vor. Aufgrund der typologischen Heterogenität unterscheidet Svoboda zwei Fazies bzw. Varianten oder „Stile“..9 Der PředmostíStil, zu dem auch die AH 6 bis 8 aus Willendorf II zählen, wird durch retuschierte Spitzklingen und ausgearbeitete marginale oder steile Retuschen an größeren Klingen sowie Abschlägen charakterisiert, während beim Dolní Věstonice-Pavlov-Stil marginale Retuschen kaum auftreten und geometrische Mikrolithen wie auch gezähnte Mikrosägen sehr häufig sind.

Im Jahre 1993 wurde das Profil aus dem Jahre 1981 erweitert (siehe Abschnitt 3) und daher eine Ausgrabung am Fundplatz Willendorf II unternommen. Das dabei geborgene und dokumentierte Steinartefaktinventar entstammt fast ausschließlich den AH 6 und 8. Ausgehend von der Proseminararbeit von Viola C. Schmid führten wir im Zuge der hier vorliegenden Arbeit eine auf einer Merkmalanalyse beruhenden technologische Untersuchung durch. Wir zielen darauf $\mathrm{ab}$, folgende Forschungsfragen zu beantworten:

- Welche technologischen und typologischen Charakteristika zeichnen sich in den Steinartefaktinventaren dieser beiden $\mathrm{AH} \mathrm{ab}$ ?

- Auf welche technoökonomischen Trends kann rückgeschlossen werden?

- Inwieweit unterscheiden bzw. ähneln sich die Inventare der beiden $\mathrm{AH}$ ?

- Finden sich Hinweise auf eine Änderung in der Nutzung bzw. Funktion der Fundstelle?

- Können weiterführende Aussagen zum Verhalten der gravettienzeitlichen WildbeuterInnen von Willendorf II bzw. auf regionaler Ebene getroffen werden?

- Was kann zur chrono-kulturellen Gliederung des Gravettien im Allgemeinen und des Pavlovien im Speziellen beigetragen werden?

\section{Lage der Fundstelle}

Die Fundstelle Willendorf II (Parzellennummer 31/3, KG Willendorf in der Wachau, MG Aggsbach, VB Krems an

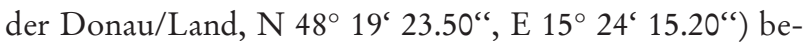
findet sich im Donautal etwa $80 \mathrm{~km}$ westlich von Wien (Abb. 1). Der ungefähr $30 \mathrm{~km}$ lange Abschnitt des Donaulaufs zwischen Melk und Krems wird als Wachau bezeichnet. Die Donau bahnte sich hier mit einem tief eingeschnittenen Tal ihren Weg durch die ins Paläozoikum datierende Böhmische Masse. Das rechte Ufer des Donautals wird durch steile, klippenartige Hänge gebildet. Das linke Ufer hingegen weist aufgrund der auflagernden

49 SvOBOda 2003

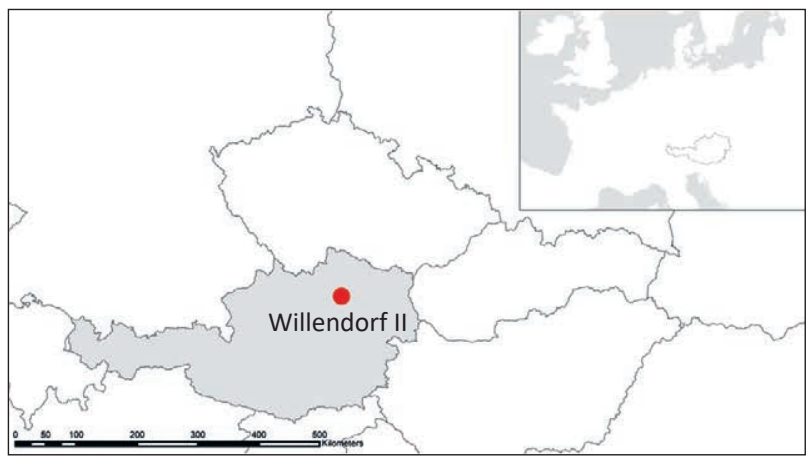

Abb. 1. Die Lage von Willendorf II (Grafik: P. R. Nigst).

Lössakkumulation flachere, abgerundete Hangrücken auf, die im Windschatten der hauptsächlich aus Westen kommenden Winde entstehen konnten. Außerdem existieren auf dieser Uferseite große Schwemmfächer, die durch Bäche im Hinterland, wie den Willendorfer Bach, entstanden. Die Bäche transportierten erhebliche Mengen an Material in das Donautal. ${ }^{50}$

Willendorf II gehört zu einer unter der Fundortsbezeichnung Willendorf zusammengefassten Fundstellengruppe von insgesamt acht Fundstellen, welche im Bereich der Katastralgemeinden Willendorf in der Wachau (Willendorf I, I-Nord, II, III, IV) und Schwallenbach (Willendorf V, VI, VII) entdeckt wurden (Abb. 2). ${ }^{51}$ Die Ablagerungen der Fundstelle Willendorf II liegen auf einer Niederterrasse der Donau. Die paläolithischen $\mathrm{AH}$ wurden in der oberen Hälfte der bis zu 20 m mächtigen Ablagerungen nachgewiesen. ${ }^{52}$

\section{Forschungsgeschichte}

\subsection{Allgemeine forschungsgeschichtliche Angaben}

Erstmalig wird die archäologische Fundstelle Willendorf II im Jahr 1889 von Ferdinand Brun erwähnt. ${ }^{53} 1890$ fanden erste Ausgrabungen geleitet von Ludwig Hans Fischer statt. ${ }^{54}$ Sowohl Fischer als auch Brun übergaben ihre Funde dem k. k. Naturhistorischen Hofmuseum (heute Naturhistorisches Museum Wien). ${ }^{55} 1907$ unternahm Rudolf Much, Sohn des österreichischen Prähistorikers Matthäus Much, in Willendorf II Grabungen. Zusammen mit den Objekten

50 Nigst et al. 2008a, 32.

51 Felgenhauer 1956-1959, 3.

52 Haesaerts et al. 1996, 27.

53 Nigst et al. 2008a, 34.

54 RutTKAY 1987, 212.

55 ANTL-Weiser 2008a, 31. 


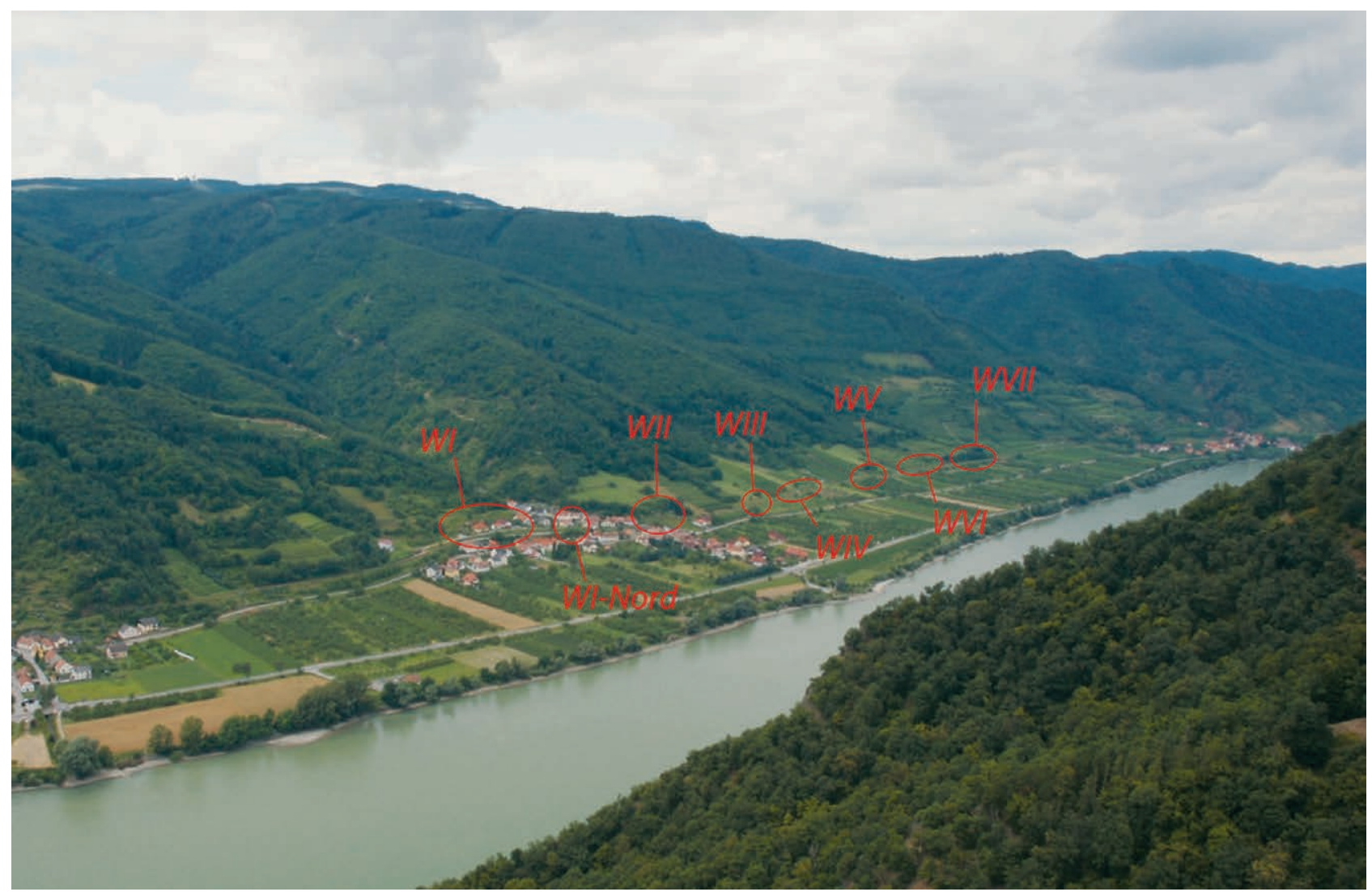

Abb. 2. Das Donautal in der Wachau im Bereich von Willendorf, Ansicht von Südosten. Rot gekennzeichnet sind die Willendorf-Stationen: Willendorf I (WI), Willendorf I-Nord (WI-Nord), Willendorf II (WII), Willendorf III (WIII), Willendorf IV (WIV), Willendorf V (WV), Willendorf VI (WVI) und Willendorf VII (WVII) (Foto: T. B. Viola, Grafik: P. R. Nigst).

der Sammlung Much wurde das Fundmaterial aus Willendorf II, zu welchem jegliche Informationen über die stratigrafische Zuordnung fehlen und welches mit einzelnen Objekten aus Willendorf I vermengt wurde, der Studiensammlung des Instituts für Ur- und Frühgeschichte der Universität Wien vermacht ${ }^{56}$ und von Richard Pittioni 1932 publiziert. ${ }^{57}$

Nach 1907 wurde bei den Bauarbeiten der Donauuferbahn eine Serie von neuen Fundstellen entdeckt, darunter Willendorf I-Nord und Willendorf III bis VII. Im Auftrag von Josef Szombathy, dem Leiter der anthropologisch-prähistorischen Sammlung der Anthropologisch-Ethnografischen Abteilung des k. k. Naturhistorischen Hofmuseums, waren Hugo Obermaier, freier Mitarbeiter des Naturhistorischen Museums, und Josef Bayer, Volontär des Naturhistorischen Museums, 1908 fast täglich vor Ort, um die Schichten und anfallenden Artefakte zu dokumentieren. ${ }^{58}$

56 Felgenhauer 1956-1959, 10.

57 PitTiONI 1932

58 ANTL-Weiser 2008a, 33.
Die ersten systematischen Ausgrabungen der Fundstelle Willendorf II begannen 1908 unter der Grabungsleitung von Szombathy. Obermaier und Bayer wurden als gleichberechtigte, örtliche Grabungsleiter eingesetzt, wobei Obermaier die wissenschaftliche Leitung innehatte. ${ }^{59} \mathrm{Wäh}$ rend der ersten Grabungskampagne wurde die komplette stratigrafische Abfolge aufgeschlossen. ${ }^{60}$ Am 7. August 1908 wurde das herausragendste Objekt, die Frauenstatuette Venus I von Willendorf, gefunden. ${ }^{61}$

Bei der zweiten Grabungskampagne von 1909 unter der Leitung von Szombathy und unter der örtlichen Grabungsleitung von Bayer stand die Erforschung der unteren Schichten im Vordergrund. Daher wurde eine Tiefengrabung durchgeführt, welche bis zu 9 m erreichte. ${ }^{62} 1912$ legte Szombathy drei weitere Tiefengrabungen bis zu $6 \mathrm{~m}$ an. ${ }^{63}$ Bayer führte die Feldarbeit in Willendorf von 1913 an fort.

\footnotetext{
59 AnTL-WEISER 2008a, 34-35.

60 AnTL-Weiser 2000-2001, 51.

61 Szombathy 1909. - AntL-Weiser 2000-2001, 40.

62 Felgenhauer 1956-1959, 12.

63 Felgenhauer 1956-1959, 12.
} 

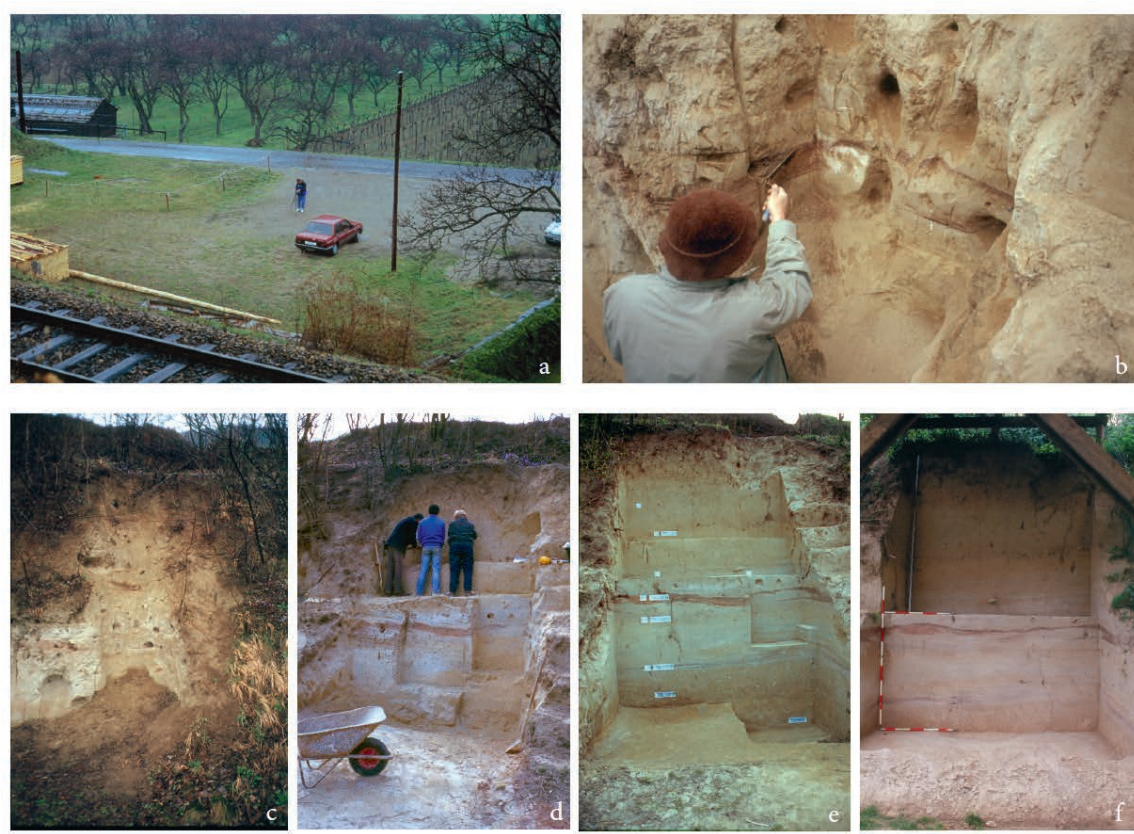

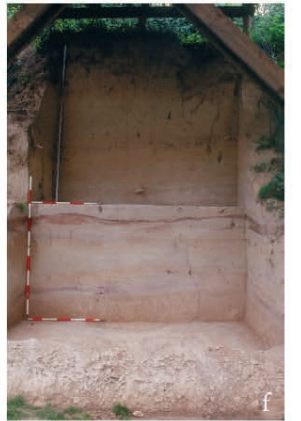

Abb. 3. Willendorf II, Grabung 1993: Ausgrabungsarbeiten 1993 und finaler Profilputz des inzwischen überdachten Schauprofils 1996. - a. Einrichtung des lokalen Messsystems von Martin Urbanek. - b. Paul Haesaerts bei der Arbeit am Profil. - c-e. Von der Öffnung des Profils von 1981 bis hin zum fertigen Profil mit Schichtkennzeichnung 1993. - f. Schauprofil mit Überdachung nach Profilputz 1996 (Fotos: G. Trnka).
Durch ihn und sein Team wurde 1913 die Grabungsfläche im Norden erweitert und die AH 4 und 5 ausgegraben. ${ }^{64}$ Außerdem wurde im weiter westlich bzw. bergwärts gelegenen Schnitt AH 9 angeschnitten. ${ }^{65}$ Nachdem Bayer Raubgrabungen gemeldet worden waren, wurde 1926 die Grabungstätigkeit in Willendorf wieder aufgenommen. ${ }^{66}$

Bei erneuten Untersuchungen 1955 durch Fritz Felgenhauer, Universität Wien, wurde unter anderem ein ca. $25 \mathrm{~m}$ langer und $3 \mathrm{~m}$ tiefer Profilgraben parallel zum alten Westprofil angelegt, um einen Überblick über die Schichtverhältnisse zu erlangen. ${ }^{67} 1981$ schließlich begann Paul Haesaerts vom Institut Royal des Sciences Naturelles de Belgique seine Feldarbeiten in Willendorf II. Er und sein Team legten ein kleines Profil an und entnahmen zahlreiche Proben. ${ }^{68}$ Im Jahr 1993 wurde im Zuge eines gemeinschaftlichen Forschungsprogramms des Institut Royal des Sciences Naturelles de Belgique und der Universität Wien die Arbeit in Willendorf II fortgesetzt (siehe Abschnitt 3.2). 1996 wurde beschlossen, das von Haesaerts erstellte Profil mit einer Dachkonstruktion zu schützen. Vor der Umsetzung wurde das ausgetrocknete Profil von einem Team um Spyridon Verginis, Universität Wien, geputzt, dabei wurden zwischen 5 und $30 \mathrm{~cm}$ Sediment entfernt sowie verschiedenste Proben entnommen. ${ }^{69}$

64 Kromer 1950, 64.

65 Felgenhauer 1956-1959, 12.

66 BAYER 1930.

67 Felgenhauer 1956-1959, 89-90.

68 Haesaerts 1990.

69 Nigst et al. 2008a, 38.
Zwischen 2005 und 2011 fanden unter der Leitung von Philip R. Nigst, Max-Planck-Institut für evolutionäre Anthropologie und Department für Archäologie der Universität Cambridge, T. Bence Viola, Department für Anthropologie der Universität Wien, und Gerhard Trnka, Institut für Ur- und Frühgeschichte der Universität Wien, im Zuge des interdisziplinären Forschungsprojekts „The Willendorf Project“ neue Grabungen statt. ${ }^{70}$ Seit 2006 wurden sechs Grabungskampagnen durchgeführt. ${ }^{71}$ Im Jahre 2012 wurde von Walpurga Antl-Weiser, Naturhistorisches Museum Wien, Marjolein D. Bosch und Philip R. Nigst, beide Universität Cambridge, das Profil aus dem Jahr 1996 erneuert. $^{72}$

\subsection{Die Ausgrabung 1993}

Nach seinem Profil von 1981 untersuchte Haesaerts gemeinsam mit Freddy Damblon, Institut Royal des Sciences Naturelles de Belgique, Margit Bachner und Gerhard Trnka, beide Institut für Ur- und Frühgeschichte der Universität Wien, die stratigrafische Abfolge der Fundstelle Willendorf II im Jahre 1993 erneut (Abb. 3). Das Profil aus dem Jahr 1981 wurde erweitert und resultierte in einem $3 \mathrm{~m}$ breiten und 5,5 $\mathrm{m}$ hohen Profil (Abb. 4). Die längslaufende Nord-Süd-Achse wurde an den vier die Parzelle 31/3 eingrenzenden Marksteinen ausgerichtet. Tatsächlich orientierten davor sowohl Bayer und Obermaier als auch Felgenhauer die Grenzen ihrer Grabungsflächen an diesen

70 Nigst et al. 2008b, 11.

71 Nigst et al. 2008a. - Nigst et al. 2008b. - NigSt et al. 2008c. Nigst et al. 2014.

72 Nigst, Antl-Weiser 2013. 


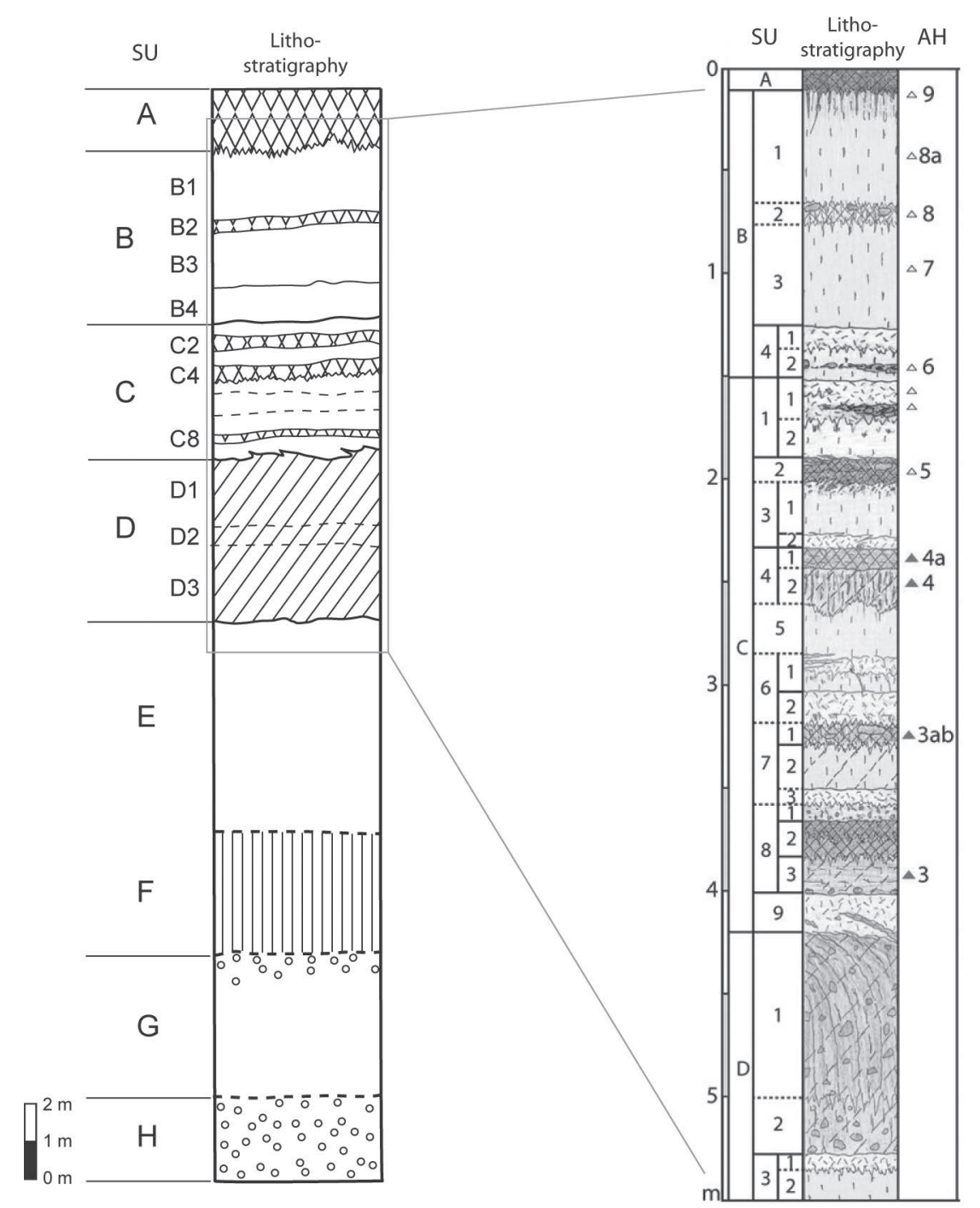

Abb. 4. Schematische stratigrafische Abfolge von Willendorf II. - Links: Stratigrafische Einheiten A bis H (A bis D beruhen auf HAEsAeRTS et al. 1996, E bis H beruhen auf Felgenhauer 1956-1959). - Rechts: Im Profil von 2011 aufgeschlossene stratigrafische Abfolge basierend auf NigST et al. 2014. - AH: Archäologischer Horizont. - SU: Stratigrafische Einheit. - Leere Dreiecke: Gravettien. - Volle Dreiecke: Aurignacien (Grafik: P. R. Nigst unter Verwendung einer Profilzeichnung von P. Haesaerts im rechten Bildteil).

Abmarkungen. Daher war eine Lokalisierung der südwestlichen Ecke des Profils 1993 exakt 21 m nördlich des Referenzpunkts 0 , der von Bayer verwendet wurde, und $20 \mathrm{~m}$ südlich des Marksteins, der die nördliche Grenze der Grabung 1955 markierte, möglich. Der Referenzpunkt O für die Höhe wurde an der Spitze des Fundaments des Bahneinschnitts nahe der südlichen Grabungsgrenze 1993 festgelegt. Diese Position dürfte mehr oder weniger mit dem Höhenreferenzpunkt 0 von Obermaier und Bayer übereinstimmen. ${ }^{73}$ Die Grabungszonen 01 bis 06 der Grabung Nigst, Viola und Trnka liegen nördlich, südlich und östlich zum Profil (Abb. 5).

73 Haesaerts et al. 1996, 27-28.
Zahlreiche Proben für verschiedene Analysen betreffend Sedimentologie, Malakologie, Paläobotanik und Radiokohlenstoffdatierung wurden genommen. ${ }^{74}$ Die Feldarbeiten dienten dazu, an einem größeren Profil die Stratigrafie und die laterale Variabilität der Ablagerungen zu untersuchen. Außerdem sollten ausreichend Holzkohleproben für Radiokohlenstoffdatierungen aus der gesamten Sequenz gesammelt werden, um neben der maßgeblichen Beschreibung der Stratigrafie eine chronologische Feingliederung zu erstellen. ${ }^{75}$

74 Frank, Rabeder 1994. - Damblon, Haesaerts, Van Der Plicht 1996. - Haesaerts et al. 1996.

75 Haesaerts et al. 1996, 28. 


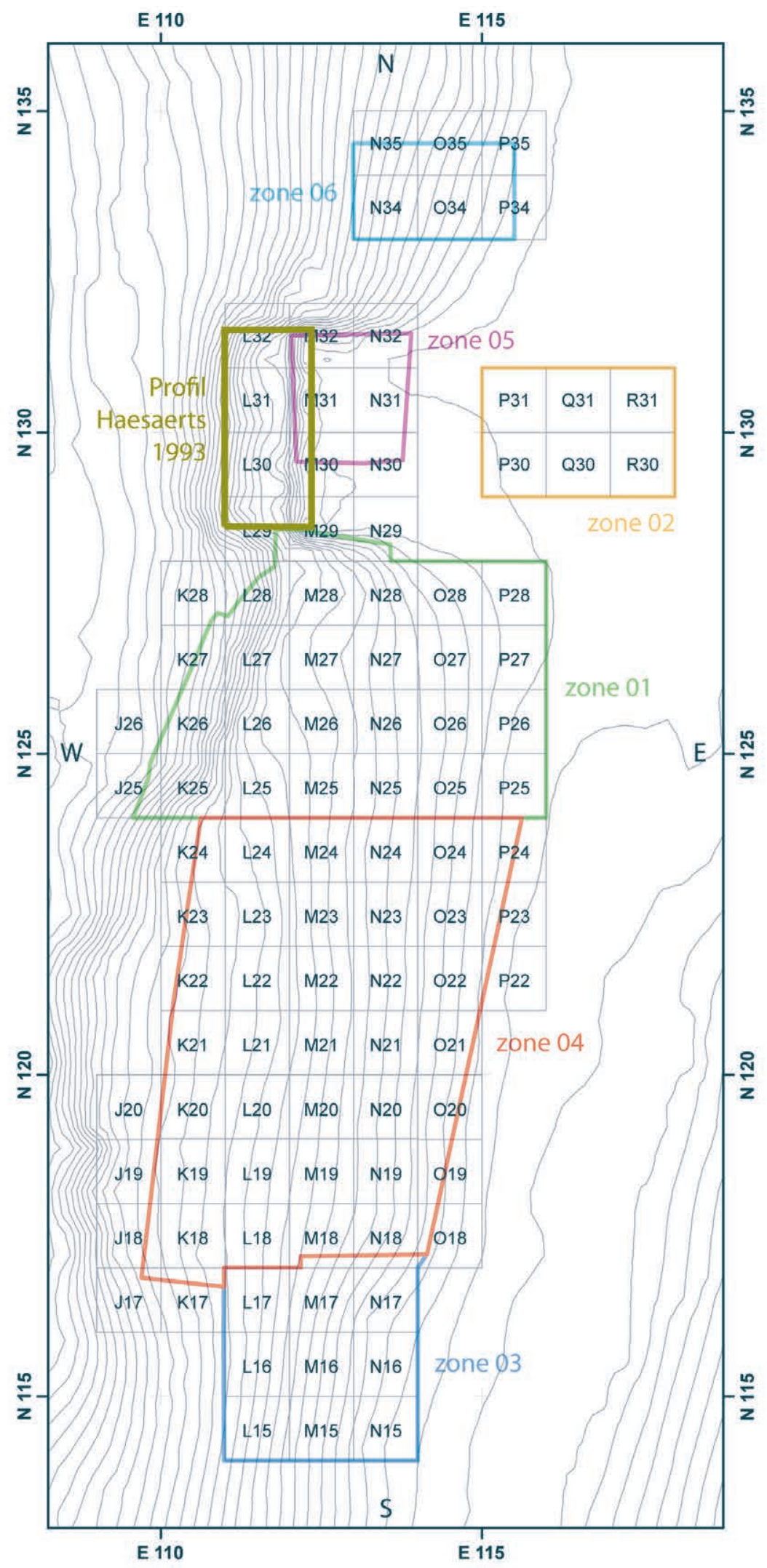

Abb. 5. Willendorf II: Grabung 2006-2011 mit Kennzeichnung der Grabungszonen 01-06 und der Lage des Profils von P. Haesaerts aus dem Jahre 1993 (Grafik: P. R. Nigst). Alle Angaben in Meter. 
Bei diesen Grabungsaktivitäten wurde das dieser Arbeit zugrundeliegende archäologische Fundmaterial von insgesamt 391 Objekten geborgen. Es wurde ein lokales Koordinatensystem verwendet. Angaben zu Koordinaten oder $\mathrm{AH}$ der Funde wurden auf den Fundzetteln festgehalten und außerdem existieren entsprechende Listen in der Grabungsdokumentation. Nicht jedem Fund wurde eine individuelle Fundnummer zugewiesen. Wenn Artefakte sich in unmittelbarer Nähe zueinander befanden, wurden sie unter ein und derselben Fundnummer aufgenommen. Die Artefakte wurden am Institut für Ur- und Frühgeschichte der Universität Wien aufbewahrt und nach unserer Analyse an die Prähistorische Abteilung des Naturhistorischen Museums Wien übergeben.

\section{Stratigrafie}

Nach den ersten Fundstellenbeschreibungen von Szombathy, Obermaier und Bayer stellte Friedrich Brandtner anhand von Bohrungen und Untersuchungen bei den Grabungen 1955 ein grundlegendes stratigrafisches System auf. ${ }^{76} \mathrm{Im}$ Folgenden wird die aktuellste, von Haesaerts vorgelegte Beschreibung der Stratigrafie von Willendorf II basierend auf seinen Feldarbeiten seit 1981 präsentiert (Abb. 4). ${ }^{77}$

Die Sequenz umfasst sechs lössige und lehmige Hauptsedimentkörper. Die untere Hälfte der Abfolge setzt sich aus zwei hellgelben Lössablagerungen zusammen, den Units $\mathrm{G}$ und $\mathrm{E}$, die durch einen rötlich braunen, teilweise umgelagerten Paläoboden, Unit F, voneinander getrennt sind. In der oberen Hälfte der Sequenz folgen die Hauptsedimente Units D, C sowie B und die moderne Humusschicht Unit A auf den in das frühe Pleniglazial fallenden Löss, Unit E. ${ }^{78}$ Der Fokus hier liegt auf Hauptsediment Unit B, aus welchem der Großteil der Artefakte der Grabung 1993 entstammt.

Unit $\mathrm{D}$ besteht aus 2,5 bis $3 \mathrm{~m}$ dicken, steinigen, heterogenen, braunen Lehm- und Lössablagerungen. Den untersten Abschnitt bildet der Löss, Subunit D3, der auf $48.500 \pm$ 800 BP (> $47.700 \mathrm{cal} \mathrm{BP})$ datiert. Dieser Löss wird von dem sandigen Lehmkörper, Subunits D2-D1, gekappt und stellt einen Paläoboden mit einem ${ }^{14} \mathrm{C}$-Alter zwischen 45.100 und 43.200 BP (48.000 und $45.600 \mathrm{cal} \mathrm{BP}$ ) dar. ${ }^{79}$

Die 2,7 m dicke Unit $\mathrm{C}$ beinhaltet eine komplexe, den oberen Teil des mittleren Pleniglazials umfassende Abfolge von hellgrauem Schluff, Subunits C9, C7-3, C6-2, C3-2

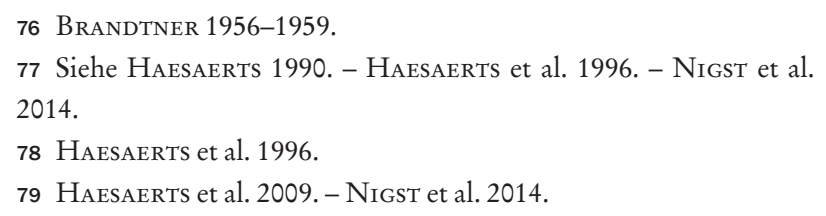

und C1-1, gelbgrauem Schluff und sandigem Schluff, Subunits C7-2, C6-1, C5, C3-1 und C1-2, einem olivgrauen, sandigen Lehm, Subunit C8-3, und von bräunlich grauen bis hin $\mathrm{zu}$ dunkelgrauen, schluffigen Bodenhorizonten, Subunits C8-2, C8-1, C7-1, C4-2, C4-1 und C2. Subunit C8-3 beinhaltet den $\mathrm{AH} 3$ mit einem Minimumalter von $39.000 \mathrm{BP}(43.500 \mathrm{cal} \mathrm{BP}) .{ }^{80}$ Subunit C7-1 ist eine leicht bioturbierte Pararendzina, die mit der menschlichen Begehung von AH 3ab um ca. 34.570 +410/-330 BP (39.500$38.700 \mathrm{cal} \mathrm{BP}$ ) übereinstimmt. Im gelbbraunen, bioturbierten Horizont mit schwarzbraunen Wurzelgängen und einer unregelmäßigen Untergrenze, Subunit C4-2, befindet sich AH 4, der zwischen 33.900 und 31.800 BP (39.20035.400 cal BP) datiert. ${ }^{81}$ Der humose Schluff darüber, Subunit C4-1, inkludiert zahlreiche Holzkohlen, die ein Alter zwischen 32.200 und $31.200 \mathrm{BP}$ (36.300-35.400 cal BP) ergaben, und Artefakte, die zu AH 4a gehören. ${ }^{82}$ Subunit C2 zeichnet sich am klarsten ab und tritt als bis zu $15 \mathrm{~cm}$ dicker, dunkelbrauner und durch Solifluktion aufgefächerter Boden mit Holzkohlenkonzentrationen auf. AH 5 wurde in dieser Subunit, die auf $30.500+900 /-800$ BP (35.50033.800 cal BP) datiert, dokumentiert. ${ }^{83}$

Bei Unit B handelt es sich um die spätpleniglaziale Lössdeckschicht, die auf einen fortschreitenden Trend zu einem kalten und trockenen Klima hinweist. ${ }^{84}$ Dieser Löss bedeckt den Tundra-Gley C1-1. In Subunit B4 befindet sich AH 6, der zwischen 27.600 und 26.500 BP (31.600-30.300 cal BP) datiert. Subunit B3 stellt eine Lössablagerung dar, die in ihrer Mitte AH 7 enthält. Im mittleren Teil von Unit B in Verbindung mit der ca. $5 \mathrm{~cm}$ dicken durchgehend hellbraunen Subunit B2, an deren Unterkante sich zahlreiche Spuren von Wurzeln abzeichnen, tritt AH 8 auf. Für B2 wurde ein Alter zwischen 25.800 und $25.400 \mathrm{BP}$ (30.800-29.200 cal BP) ermittelt. In Subunit B1, einem blassgelben, homogenen Löss, wurde erstmalig beim Profilputz 1993 anhand von Holzkohlen AH 8a erkannt. Bei den Grabungsarbeiten 2006 wurden in Zone 01 bei Arbeiten am Profilabbruch der Altgrabungen einige Steinartefakte und Knochen aus dieser neuen Schicht geborgen. AH 9 wurde seit den Feldarbeiten von Felgenhauer 1955 nicht mehr angetroffen. ${ }^{85}$ Dieser AH dürfte sich im obersten Bereich von Subunit B1 befunden haben. ${ }^{86}$

\footnotetext{
80 Nigst et al. 2014.

81 Nigst et al. 2008b. - Nigst, Haesaerts 2012. - Nigst et al. 2014.

82 Nigst et al. 2014.

83 Haesaerts et al. 1996. - Nigst et al. 2014.

84 Frank, Rabeder 1994. - Haesaerts et al. 1996.

85 Haesaerts et al. 1996. - Nigst et al. 2008a, 45-46.

86 Haesaerts et al. 1996, 32.
} 
Der ca. $90 \mathrm{~cm}$ dicke rezente Bodenhorizont Unit A, der sich an den Verlauf der Hangtopografie hält, beinhaltet Holzkohlefragmente und moderne Keramik. Bioturbation und humose Infiltration, die von diesem Horizont ausgehen, wirken sich in den ersten $20 \mathrm{~cm}$ auf den darunterliegenden Löss Subunit B1 und damit sehr wahrscheinlich auf AH 9 aus. ${ }^{87}$ Auch im Fundmaterial der Profilarbeiten 1993 befinden sich vier Keramikfragmente, die aus dieser Unit bzw. dem gestörten, obersten Bereich von Subunit B1 stammen dürften.

\section{Archäologische Abfolge}

In diesem Abschnitt sollen kurz alle $\mathrm{AH}$ angesprochen werden.

AH 1: Seit der ersten Beschreibung 1909 wurde AH 1 nicht mehr angetroffen. Aufgrund des undiagnostischen Inventars konnte keine techno-typologische Zuordnung erfolgen. ${ }^{88}$

AH 2: Dieser Horizont wurde 1909 erstmalig beschrieben und die Steinartefakte aus diesem AH wurden lange Zeit als nicht diagnostisch angesehen, können aber höchstwahrscheinlich dem frühen Jungpaläolithikum zugeschrieben werden. ${ }^{89} \mathrm{Als}$ Begründung, keine konkrete chrono-kulturelle Zuweisung vornehmen zu können, verwiesen die meisten Autoren auf die geringe Anzahl an Artefakten. Kürzlich im Keller des Naturhistorischen Museums Wien entdeckte hölzerne Transportkisten der Grabungen 1908 und 1909 enthielten zusätzliche Steinartefakte aus den AH 2 und 3, die von den Grabungen 1908/1909 stammen. ${ }^{90}$ Somit liegen nun für AH 2 mehr als 1000 Stücke vor, die von Nigst analysiert und als frühes Jungpaläolithikum, potentiell Szeletien, klassifiziert wurden..$^{91}$

AH 3: AH 3 kann als frühes Aurignacien angesprochen werden und wurde erstmalig 1908 und 1909 ausgegraben. ${ }^{92}$ Die Schicht wurde bei der Grabung 1955 in einem kleinen Bereich dokumentiert und konnte auch bei den Grabungen unter der Leitung von Nigst, Viola und Trnka nachgewiesen werden (Abb. 4). ${ }^{93}$

$\mathrm{AH}$ 3ab: Die Beschreibung dieses Horizontes erfolgte erstmals bei der Grabung 2006. Bei dem Inventar handelt es sich um ein Aurignacien. ${ }^{94}$

87 HAESAERTS et al. 1996, 30.

88 Hahn 1977. - TeysSAndier 2003. - Nigst et al. 2008a, 47.

89 Hahn 1977. - Haesaerts, Teyssandier 2003. - Teyssandier

2003. - Nigst 2006. - Nigst, Haesaerts 2012.

90 Nigst 2004. - Nigst et al. 2008a, 44. - NigSt et al. 2014.

91 Nigst 2012.

92 Nigst et al. 2014.

93 Nigst et al. 2008a, 47. - Nigst et al. 2008b, 10. - Nigst et al. 2014.

94 Nigst et al. 2008a, 47. - Nigst et al. 2014, Supporting Information 3.
AH 4: Dieser Horizont wird dem Aurignacien mit zahlreichen Kiel- und Nasenkratzern zugeordnet und wurde erstmalig 1908 sowie seither unter anderem auch bei den aktuellsten Grabungen angetroffen. Die Feldarbeiten 1993 lieferten zwei Steinartefakte aus dieser Schicht, wobei eines davon leider verschollen ist. Holzkohlen kommen in AH 4 zahlreich und gut erhalten vor. ${ }^{95}$

AH 4a: Das Steinartefaktinventar dieser Schicht wird wie AH 4 einem klassischen Aurignacien zugeordnet. ${ }^{96}$

AH 5: Die Schicht wurde auch 1908 bereits erkannt und seither bei mehreren Grabungen beschrieben. Dieses Inventar umfasst die älteste gravettienzeitliche Besiedlung Willendorfs II. ${ }^{97}$

AH 6: Dieser AH wurde dem Gravettien zugeschrieben. ${ }^{98}$ 27,6 \% des hier analysierten lithischen Materials entstammen diesem Horizont.

AH 7: Dieser AH wurde ebenfalls dem Gravettien zugeordnet. ${ }^{99}$

AH 8: Seit der ersten systematischen Grabung 1908 wurde AH 8 bei verschiedensten Grabungen angetroffen. Dieser Horizont wurde als Gravettien angesprochen. ${ }^{100}$ 69,4 \% des Inventars der Feldarbeiten 1993 stammen aus AH 8.

AH 8a: Hierbei dürfte es sich um eine gravettienzeitliche Besiedlung handeln. ${ }^{101}$

$\mathrm{AH}$ 9: Dieses Inventar wurde aufgrund der charakteristischen Kerbspitzen der spätgravettienzeitlichen Phase Willendorfien-Kostienkien zugeordnet. ${ }^{102}$ Dieser AH wurde weder bei den aktuellsten Grabungen noch bei den Profilarbeiten 1981/1993 angetroffen. ${ }^{103}$

\section{Methodik}

Die Merkmalanalyse stellt ein geeignetes Analysewerkzeug dar, um die Schlagtechnik und die Abbaustrategien der paläolithischen WildbeuterInnen zu rekonstruieren. Bei der Merkmalanalyse werden alle Steinartefakte erfasst und kein Steinartefakt bevorzugt behandelt. ${ }^{104}$ Durch die Analyse

95 Nigst et al. 2008a, 46-47. - Nigst et al. 2008b, 10. - Nigst 2012. - Nigst et al. 2014, Supporting Information 3.

96 Nigst et al. 2014.

97 Felgenhauer 1956-1959. - Broglio, Laplace 1966. - Hahn 1977. - OtTe 1981. - KozŁowski 1986. - OtTe 1991. - Teyssandier 2007. - Nigst et al. 2008a. - Moreau 2012.

98 Nigst et al. 2008a, 46.

99 Nigst et al. 2008a, 46.

100 Nigst et al. 2008a, 46.

101 Nigst et al. 2008a, 45.

102 Svoboda 2007, 207. - Nigst et al. 2008a, 45.

103 Haesaerts et al. 1996, 32. - Nigst et al. 2008a, 45.

104 Tostevin 2000a. - Tostevin 2003a. - Tostevin 2011. - Nigst

2012. - Tostevin 2012. - Nigst 2014. - Nigst 2019. 
spezifischer Merkmale wird versucht, die Organisation der lithischen Technologie von der Auswahl des Rohmaterials, der Kernpräparation für den Grundformenabbau, der Platzierung der Retusche und der Nachschärfung bis hin zur letztendlichen Verwerfung der Steinartefakte zu dokumentieren. So kann am Inventar mit Hilfe der Merkmalanalyse eine Identifizierung bestimmter wiederkehrender Merkmalskombinationen erfolgen, die Aufschluss über Entscheidungen, die die paläolithischen Menschen im Zuge der chaîne opératoire trafen, und damit damaliger Verhaltensmuster geben. ${ }^{105}$ Anhand dieser Methodik können somit technologische Trends untersucht und durch Quantifizierung untermauert werden. ${ }^{106}$ Unter Steintechnologie wird in diesem Zusammenhang jegliche Handlung des Menschen verstanden, die in den Herstellungsprozess und die Verwendung eines lithischen Objekts involviert ist. Nach Abschluss der Aufnahme verlief die Analyse der Merkmale durch univariate Tests (t-Tests, Shapiro-Wilk-Test, Chi²-Test, Wilcoxon-Mann-Whitney-Test) und Vergleiche von Paaren von Grundformen- und Kernmerkmalen. Die statistischen Tests wurden mit der Software Past ${ }^{107}$ und $\mathrm{R}^{108}$ durchgeführt. Scatterplots und Boxplots wurden mit der R-Erweiterung ggplot2 realisiert. ${ }^{109}$ Zusätzlich zur Erschließung der technologischen Abläufe, die hinter den Artefakten stecken, ist so ein möglichst objektives Arbeiten gewährleistet und es wird eine konkrete Nachvollziehbarkeit geschaffen. ${ }^{110}$

Zur Umsetzung der Datenaufnahme mittels eines merkmalanalytischen Aufnahmesystems wurde eine Datenbank (Software: Microsoft Access) erstellt. Die Datenerfassung erfolgte mittels der Software E4. ${ }^{111}$ Die metrischen Angaben wurden mit einer digitalen Schiebelehre direkt in die Datenbank transferiert. ${ }^{112}$

Bevor die Aufnahme allerdings beginnen konnte, musste jedem Artefakt eine eindeutige Inventarnummer zugewiesen werden, da, wie oben bereits angesprochen, Funde, die sich in unmittelbarer Nähe zueinander befanden, unter einer Fundnummer zusammengefasst wurden. Von diesen „Sammelfunden“ erhielt somit jedes Objekt eine individuelle Subnummer.

105 HAHN 1993, 74-75.

106 Movius et al. 1968. - SCHÄFEr 1988. - AuffERMAnN et al. 1990. Tostevin 2000b, 96. - Adler 2002. - Nigst 2012. - Tostevin 2012. 107 Hammer, Harper, Ryan 2001.

108 R CORE TEAM 2018.

109 WICKHAM 2016.

110 Movius et al. 1968. - SCHÄFEr 1988. - AuffERMANn et al. 1990. Tostevin 2000b, 96. - Adler 2002. - NigSt 2012. - Tostevin 2012. 111 http://www.oldstoneage.com/software/e4.shtml (letzter Zugriff 18.9.2019).

112 Mcpherron, Dibble 2002, 66.
Zusätzlich wurde systematisch untersucht, ob Zusammenpassungen im Inventar vorliegen und um welche Art der Zusammenpassung es sich handelt, da so ergänzende Informationen zur Technologie gewonnen werden können sowie Aussagen zur räumlichen Verteilung innerhalb der Fundstelle und stratigrafischen Integrität der Fundschichten möglich sind. ${ }^{113}$

\subsection{Merkmalanalytisches Aufnahmesystem}

Als Vorlage für das hier verwendete merkmalanalytische Aufnahmesystem diente jenes von Nigst, ${ }^{114}$ daneben soll an dieser Stelle auch auf weitere ähnliche Attributlisten aus der Literatur verwiesen werden. ${ }^{115}$ Im folgenden Abschnitt werden ausschließlich jene Attribute aufgelistet, die nicht identisch mit der Attributliste von Nigst ${ }^{116}$ sind.

Als Erstes wurden Fundnummer und Schicht in die Datenbank aufgenommen. Zu den Schichtangaben sei angemerkt, dass sowohl die Schichtzuweisungen, die direkt bei der Grabung erfolgten, als auch die aus den Messdaten rekonstruierten Schichtzugehörigkeiten angegeben wurden.

Wie bei Nigst ${ }^{117}$ beschrieben, wurde eine makroskopische Rohmaterialienbestimmung durchgeführt, um anhand von Fossilieneinschlüssen, Farbe, Struktur der natürlichen Oberflächen (einschließlich „Kortex“ im Fall organisch gebildeter $\mathrm{SiO}_{2}$-Modifikationen wie z. B. Hornstein, Feuerstein oder Radiolarit), Granularität und Homogenität eine Zuweisung zu einer Rohmaterialgruppe, die geläufigen mineralogischen und geologischen Definitionen entspricht, zu treffen. ${ }^{118}$ Lokale Rohmaterialien beziehen sich auf Gesteine, die im Umkreis von $10 \mathrm{~km}$ um die Fundstelle in den Donauschottern vorkommen. Hingegen wurden nicht-lokale Rohstoffe von einer Distanz größer als $10 \mathrm{~km}$ zur Fundstelle transportiert. ${ }^{119}$

Eine weitere Variable gibt an, ob eine natürliche, post-depositionelle Veränderung der Oberfläche des Steinartefakts, eine sogenannte Patina, beobachtet wurde. Zu den Faktoren, die Art und Häufigkeit der Bildung der chemisch induzierten Patina verursachen, zählen die Zusammensetzung und die Oberflächenstruktur des Gesteins genauso wie der $\mathrm{pH}$-Wert, die Temperatur, die Feuchtigkeit und

\footnotetext{
113 Cziesla 1990.

114 Nigst 2012

115 Siehe zum Beispiel Auffermann et al. 1990. - Отт 1996. Tostevin 2000a.

116 Nigst 2012.

117 Nigst 2012.

118 GötZinger 2006. - Ziehaus 2007. - Binsteiner, Eisner, ANTLWeiser 2008. - Brandl et al. 2014. - Moreau, Brandl, Nigst 2016. - Thomas, Brande, Simon 2016.

119 Moreau, Brandl, Nigst 2016.
} 
die chemische Zusammensetzung der umgebenden Matrix. Die Patina kann somit Informationen zu natürlichen, post-depositionellen Prozessen, welchen die Steinartefakte ausgesetzt waren, liefern. ${ }^{120}$ Aber anhand der Patina kann in seltenen Fällen auch auf das Eingreifen des Menschen geschlossen werden, beispielsweise wenn ein einzelnes Artefakt Unterschiede bei der Patinierung zwischen den originalen und später dazugekommenen Negativen aufweist. In diesem Fall handelt es sich um eine sogenannte Doppelpatina, die darauf hindeutet, dass dieses Objekt zur Wiederverwendung aufgesammelt wurde. ${ }^{121}$

Zudem wurde angegeben, ob eine bei den Artefakten aus Willendorf II häufig auftretende Versinterung nachgewiesen wurde.

Die Artefaktklassendefinitionen folgen de Sonneville-Bordes, ${ }^{122}$ Movius et al. ${ }^{123}$ und Hahn. ${ }^{124}$ Die sogenannten Klingenabschläge, die zu kurz geratene Klingen darstellen könnten, wurden trotz ihres regelmäßigen, länglichen Charakters zu den Abschlägen gezählt, ${ }^{125}$ was somit der Vorgangsweise von Nigst ${ }^{126}$ entspricht.

Bei den metrischen Angaben der verschiedenen Grundformen wurden Klingen und Lamellen sowie Abschläge und Absplisse wegen der geringen Stückzahl zusammengefasst. Bei den Stücken, deren Länge und Breite vollständig erhalten war, wurde zusätzlich die maximale Dimension gemessen, welche unabhängig von der Achse des Stückes die Distanz zwischen den beiden äußersten Punkten darstellt. ${ }^{127}$

Aufgrund der Schlagmerkmale Lippe, Bulbus, Schlagnarbe, Kegel und Schlagpunkt sowie der Art und Form des Schlagflächenrestes, der Schlagflächenrest-Dicke, der Präparation der Schlagflächenkante und dem Abbauwinkel kann tendenziell angegeben werden, welche Schlagtechnik am wahrscheinlichsten verwendet wurde. Exakte Aussagen $\mathrm{zu}$ treffen ist keinesfalls möglich. ${ }^{128}$ Für die Bestimmung wird hier vor allem auf die von Jacques Pelegrin ${ }^{129}$ publizierten Beschreibungen für den direkten, harten, den direkten, weichen, mineralischen und den direkten, weichen, organischen Schlag zurückgegriffen. So gelten für den direkten, harten Schlag ein zumindest mehrere Millimeter breiter, glatter oder facettierter Schlagflächenrest, die Ausprägung

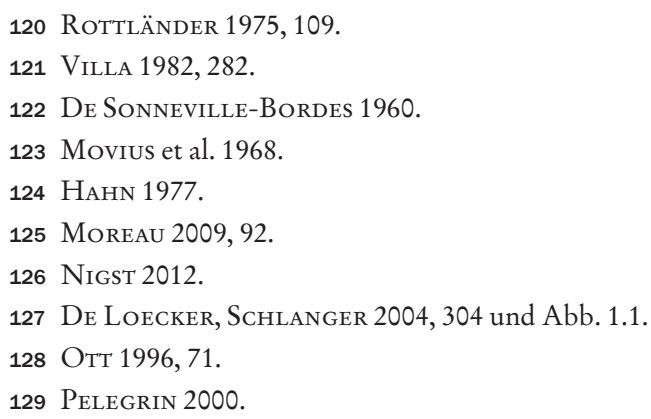

des Schlagpunktes, ein Abbauwinkel von 60 bis zu $90^{\circ}$ und das häufige Auftreten von dünnen, dichtgedrängten Wallnerlinien in den ersten Millimeter als typisch, außerdem weisen die Produkte immer eine gewisse Dicke auf. Für den direkten, weichen, mineralischen Schlag sind eine fehlende Lippe, lineare oder punktförmige Schlagflächenreste, eine sorgfältige, dorsale Reduktion und ausgeprägte konzentrische Wallnerlinien im Bulbusbereich sowie der sogenannte esquillement $d u$ bulbe, $d$. h. eine den Bulbusbereich betreffende Aussplitterung des Schlagflächenrestes charakteristisch. Der direkte, organische Schlag zeichnet sich durch eine nicht allzu ausgeprägte Schlagflächenrest-Dicke, den kontinuierlichen Verlauf der Lippe, einen Abbauwinkel unter $80^{\circ}$ sowie die dorsale Reduktion aus. Doch bei dem vorliegenden Inventar stellte sich einerseits die äußerst geringe Stückzahl als problematisch für die Bestimmung der Schlagtechnik dar und andererseits wurden bis zum derzeitigen Stand der Forschung noch keine Versuche an den in Willendorf vorkommenden Rohmaterialien unternommen, die aufzeigen würden, welche charakteristischen Merkmalskombinationen diese Rohmaterialien bei den verschiedenen Schlagtechniken aufweisen bzw. ob die Merkmale an diesen Rohmaterialien eine Differenzierung der unterschiedlichen Schlagtechniken zulassen.

Bei der Angabe zu Kantenbeschädigungen wurden auch die Ausgesplitterten Stücke angeführt, da keine intentionelle Modifikation angebracht wurde, sondern im Zuge des Gebrauchs Aussplitterungen entstanden. ${ }^{130}$

Vor allem aus Gründen der Vergleichbarkeit - auch mit anderen relevanten Arbeiten zu Willendorf ${ }^{131}$ - wurde neben der Beschreibung von Position, Lage, Art, Form, Länge und Höhe der intentionellen Modifikationen auf die gängigen Werkzeugtypenbezeichnungen nicht verzichtet. ${ }^{132} \mathrm{Da}$ es sich bei den Ausgesplitterten Stücken aber dennoch per definitionem um einen Werkzeugtyp handelt, werden sie hier genauso angeführt, von der Angabe nach der Erhaltung des Werkzeugtyps allerdings ausgenommen. ${ }^{133}$ Falls weitere, noch nicht angeführte, markante Aspekte an einem Stück auffielen, wurden diese abschließend unter „Bemerkungen“ festgehalten.

\section{Fundmaterial}

Bei der Ausgrabung 1993 wurden 391 Funde geborgen. Das Fundmaterial setzt sich aus 308 geschlagenen Steinartefakten, vier Schlagsteinen, 48 Manuporten, acht Ockerstücken,

130 Hahn 1977, 62.

131 Siehe Felgenhauer 1956-1959. - Broglio, Laplace 1966. Otte 1981. - Svoboda 1994a. - Svoboda 1996. - Moreau 2009. 132 Siehe de Sonneville-Bordes 1960. - Hahn 1977. - Hahn 1993. 133 Hahn 1977, 62. 


\begin{tabular}{|c|c|c|c|c|c|c|c|c|c|c|}
\hline \multirow[t]{2}{*}{ Fundart } & \multicolumn{2}{|c|}{ AH 4} & \multicolumn{2}{|c|}{ AH 6} & \multicolumn{2}{|c|}{ AH 8} & \multicolumn{2}{|c|}{ Modern } & \multicolumn{2}{|c|}{ Gesamt } \\
\hline & $\mathrm{n}$ & $\%$ & $\mathrm{n}$ & $\%$ & $\mathrm{n}$ & $\%$ & $\mathrm{n}$ & $\%$ & $\mathrm{n}$ & $\%$ \\
\hline $\begin{array}{l}\text { Geschlagene } \\
\text { Steinartefakte }\end{array}$ & 2 & $100 \%$ & 102 & $94,4 \%$ & 204 & $74,7 \%$ & - & - & 308 & $78,8 \%$ \\
\hline Schlagsteine & - & - & - & - & 4 & $1,5 \%$ & - & - & 4 & $1 \%$ \\
\hline Manuporte & - & - & - & - & 45 & $16,4 \%$ & 3 & $37,5 \%$ & 48 & $12,3 \%$ \\
\hline Ocker & - & - & 4 & $3,7 \%$ & 4 & $1,5 \%$ & - & - & 8 & $2 \%$ \\
\hline Knochen & - & - & 2 & $1,9 \%$ & 16 & $5,9 \%$ & 1 & $12,5 \%$ & 19 & $4,9 \%$ \\
\hline Keramik & - & - & - & - & - & - & 4 & $50 \%$ & 4 & $1 \%$ \\
\hline Gesamt & 2 & $100 \%$ & 108 & $100 \%$ & 273 & $100 \%$ & 8 & $100 \%$ & 391 & $100 \%$ \\
\hline
\end{tabular}

Tab. 1. Willendorf II: Funde der Ausgrabung 1993.

19 Knochen und vier Keramikfragmenten zusammen (Tab. 1). Die Mehrheit der analysierten Stücke (66,2 \%) kommt aus AH 8 (Tab. 1). Die Steinartefakte stammen aus den $\mathrm{AH}$ 4, 6 und 8. Ein Großteil der Funde weist eine für die Fundstelle typische Versinterung auf. Nur eines der beiden Artefakte aus AH 4 wurde aufgenommen, da das andere mit Fundnummer 173-2 leider nicht auffindbar war.

\subsection{Lithisches Rohmaterial}

\subsubsection{Rohmaterialien und Rohmaterialvorkommen}

Quarzit: Bei den lokal vorkommenden Quarziten handelt es sich um eine heterogene Materialgruppe aus echten Quarziten der metamorphen Gesteinsserien der Böhmischen Masse und anderen Kieselgesteinen Niederösterreichs. Vielfach weisen die unterschiedlichen Gesteinstypen neben einem weiten Farbspektrum auch charakteristische Bänderungen und Schlieren auf.

Quarz: Dieser Rohstoff stellt die kristalline Form von $\mathrm{SiO}_{2}$ dar und konnte in den Donauschottern aufgefunden werden.

Jaspis: Dieses Gestein, das ähnlich wie Chalcedon eine feinkörnige Modifikation von Quarz darstellt, wird im nördlichen Waldviertel und dem Dunkelsteiner Wald angetroffen. Chalcedon: Diese krypto- bis mikrokristalline Form des Tiefquarzes kommt im nördlichen Waldviertel und im Dunkelsteiner Wald vor.

Hornstein (Feuerstein): Der biogen gebildete Hornstein, dessen Kieselsäure von den Skelettresten von Kieselschwämmen stammt, konnte lokal in den Donauschottern gewonnen werden. Er kann aus Karbonatgesteinen der Klippenzonen und aus den Nördlichen Kalkalpen („Hornstein alpin“) stammen. Doch gibt es auch anderen nicht-lokalen Hornstein, den Mährischen Jurahornstein, welcher aus Lagerstätten in den Jurasedimentgesteinen in Mähren entstammt und daher aus Entfernungen zwischen 110 und $140 \mathrm{~km}$ zur Fundstelle transportiert werden musste.

Radiolarit alpin: Dieses organogene Kieselgestein wird überwiegend aus den Kieselskeletten von Radiolarien, marinen Einzellern, aufgebaut. Auch dieses Rohmaterial kann lokal in den Donauschottern aufgesammelt werden, doch andere potentielle Vorkommen repräsentieren die Klippenzonen der Flyschzone, die Kalkvoralpen und die mesozoischen Sedimente der Weißen Karpaten im mährisch-slowakischen Grenzgebiet.

Kieselkalk: Dieser lokale Rohstoff wird auch Kalkstein mit kieseliger Bindung genannt. Er weist oftmals eine typische netzartige Patina auf und kann aus den Nördlichen Kalkalpen stammen.

Baltischer Feuerstein: Der Terminus „baltischer Feuerstein “ steht für glazigene, erratische Feuersteine, die in den Schotterkörpern der Moränen des Pleistozäns während der Vorstöße des nordischen Eisschildes u. a. bis Nordböhmen, Nordmähren und Südwestpolen transportiert worden sind. Die primären Herkunftsgebiete liegen in den nordischen Kreideformationen. Charakteristisch ist eine weiße Patina, welche im hier behandelten Inventar alle Artefakte aus Feuerstein aufweisen. Oftmals zeigt der baltische Feuerstein im Durchlicht eine gewisse Transparenz. Wenn Kortex vorliegt, dann ist diese sandig braun bis kreidig weiß und zeigt häufig Schleifspuren, Schrammen oder eine Politur an der Oberfläche, wie sie bei glazialen Geschieben auftreten. Die vielfach sehr einschlussreichen glazialen Feuersteine sind homogen, oft glasartig und kompakt. Als die zu Willendorf nahegelegenste Lagerstätte von baltischem Feuerstein mit einer Entfernung von $230 \mathrm{~km}$ gilt das Gebiet der Mährischen Pforte.

Amphibolit: Das vorwiegend aus Amphibolen und Plagioklas bestehende metamorphe Gestein stammt aus der Böhmischen Masse (Waldviertel und Dunkelsteiner Wald). 
Glimmerschiefer: Das metamorphe Gestein stammt aus der Böhmischen Masse oder aus dem südwestmährischen Gebiet um Znojmo.

Serpentinit: Das metamorphe Gestein aus Pyroxen, Peridotit und Serpentinmineralen kommt in alpinen Serien und in der Böhmischen Masse vor.

Granulit: Dieses metamorphe Gestein wird ebenso in der Böhmischen Masse angetroffen. Das nächstgelegene Vorkommen liegt im Dunkelsteiner Wald.

Granit: Dieses quarzreiche magmatische Tiefengestein tritt in der Böhmischen Masse auf.

Marmor: Dieses metamorphe Karbonatgestein stammt aus der Böhmischen Masse.

Quarz-Sandstein: Dieses Sedimentgestein, vorwiegend aus Quarzkomponenten, wird im Einzugsgebiet der Donau, in der Flyschzone und in neogenen Ablagerungen angetroffen. Kalksandstein: Sandstein mit Kalkanteil als Bindemittel, stammt aus neogenen Ablagerungen der Molassezone oder aus miozänen Beckensedimenten.

Hämatitkonglomerat, Ocker: Bei diesen Rohmaterialien handelt es sich um Verwitterungs- und Anreicherungsprodukte mit hohem Eisenoxidanteil, die aus alpinen Serien und der Böhmischen Masse eingebracht wurden. Ocker besteht hauptsächlich aus „Limonit“ (FeOOH) und Tonmineralen, während „Rötel“ meist aus feinkörnigem Hämatit $\left(\mathrm{Fe}_{2} \mathrm{O}_{3}\right)$ besteht. Ocker/Limonit kann bei etwa $350-400^{\circ} \mathrm{C}$ zu Rötel/ Hämatit gebrannt werden.

\subsubsection{Rohmaterial in $\mathrm{AH} 4$}

Die Lamelle aus AH 4 wurde aus Radiolarit gefertigt (Tab. 2). Da aus diesem AH nur dieses eine Stück vorliegt und daher die Aussagemöglichkeiten sehr begrenzt sind, wurde davon abgesehen, im Folgenden weiter darauf einzugehen.

\subsubsection{Rohmaterialien der Steinartefakte des AH 6}

AH 6 ist gekennzeichnet durch einen hohen Anteil an Hornsteinen (58,8\%), gefolgt von der Gruppe der baltischen Feuersteine (27,5\%) und den Radiolariten (6,9 \%) (Tab. 2). Diese drei größten Rohmaterialklassen umfassen mehr als $90 \%$ des Inventars. Der baltische Feuerstein wurde im Gegensatz zu den lokalen Hornsteinen und Radiolariten aus einer größeren Entfernung (mindestens $230 \mathrm{~km}$ ) importiert.

Es liegen keine Kerne aus AH 6 vor. Abschläge aus allen Rohmaterialien kommen vor (Tab. 3). Für die Produktion von Klingen hingegen wurden ausschließlich die drei größten Rohmaterialklassen herangezogen. Da aber insgesamt nur wenige Klingen vorliegen, kann dies auch ein Effekt der geringen Stückzahl sein, dass gerade bei den drei häufigsten

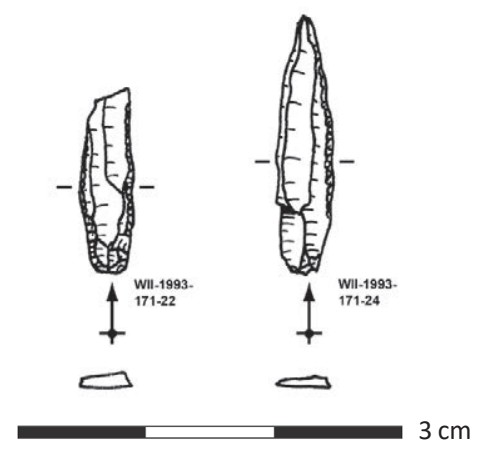

Abb. 6. Willendorf II, AH 6: Rückengestumpfte Elemente. - WII1993-171-22, Lamelle mit beidseitigem Rücken. - WII-1993-171-24, Lamelle mit einfachem Rücken (Zeichnung: F. Brodbeck).

vorkommenden Rohmaterialien diese Grundformen auftreten. Zur Gewinnung von Lamellen, die als Ausgangsformen für die im Gravettien typischen Rückenelemente gelten, wurden ausschließlich der alpine Hornstein und der baltische Feuerstein herangezogen (Tab. 3). Die zwei Rückenelemente aus dem Werkzeugbestand des AH 6 (Abb. 6) wurden aus Hornstein und aus Feuerstein gefertigt (Tab. 4). Insgesamt machen der alpine Hornstein und der baltische Feuerstein jeweils den Hauptanteil der Werkzeugtypen aus, während Radiolarit und Quarzit in geringem Maße vertreten sind (Tab. 4). Bei Hornstein und baltischem Feuerstein wurden ausschließlich Klingen und Lamellen als Grundformen zur Werkzeugherstellung verwendet, bei Radiolarit und Quarzit jeweils ein Abschlag.

Aus AH 6 gibt es bei den verschiedenen Rohmaterialien keine Grundform, die dorsal komplett mit natürlichen Oberflächen bedeckt ist. 83,3\% der Grundformen aus alpinem Hornstein und 57,1\% derer aus baltischem Feuerstein weisen überhaupt keine Bedeckung mit natürlichen Oberflächen auf (Tab. 5). Aufgrund dieses niedrigen Anteils an natürlichen Oberflächen bei diesen Rohmaterialien wird angenommen, dass bereits vor dem Transport zur Fundstelle eine Vorpräparation durchgeführt wurde. Entweder wurde die Qualität des Rohmaterials an der Rohstoffquelle direkt getestet, damit eine Selektion der hochwertigeren Rohmaterialknollen stattfinden konnte, oder durch den Austausch mit anderen Gruppen wurden bereits erprobte und reduzierte Knollen erworben, oder die Gruppe startete den Abbau der Knolle am vorhergehenden Lagerplatz und transportierte diese danach mit sich. Radiolarit hingegen zeichnet sich durch einen höheren Anteil an natürlichen Oberflächen aus, wobei die Stückzahl zu gering ist, um eindeutige Aussagen treffen zu können. Dieses Material dürfte somit ohne wesentliche Präparation in die Fundstelle eingebracht worden sein. 


\begin{tabular}{|c|c|c|c|c|c|c|c|c|}
\hline \multirow[t]{2}{*}{ Rohmaterial } & \multicolumn{2}{|c|}{ AH 4} & \multicolumn{2}{|c|}{ AH 6} & \multicolumn{2}{|c|}{ AH 8} & \multicolumn{2}{|c|}{ Gesamt } \\
\hline & $\mathrm{n}$ & $\%$ & $\mathrm{n}$ & $\%$ & $\mathrm{n}$ & $\%$ & $\mathrm{n}$ & $\%$ \\
\hline Quarzit & - & - & 1 & $1 \%$ & 84 & $41,2 \%$ & 85 & $27,7 \%$ \\
\hline Hornstein alpin & - & - & 60 & $58,8 \%$ & - & - & 60 & $19,5 \%$ \\
\hline Kieselkalk & - & - & - & - & 49 & $24 \%$ & 49 & $16 \%$ \\
\hline Baltischer Feuerstein & - & - & 28 & $27,5 \%$ & 7 & $3,4 \%$ & 35 & $11,4 \%$ \\
\hline Jaspis & - & - & - & - & 34 & $16,7 \%$ & 34 & $11,1 \%$ \\
\hline Radiolarit & 1 & $100 \%$ & 7 & $6,9 \%$ & 14 & $6,9 \%$ & 22 & $7,2 \%$ \\
\hline Quarz & - & - & - & - & 5 & $2,5 \%$ & 5 & $1,6 \%$ \\
\hline Granulit & - & - & - & - & 4 & $2 \%$ & 4 & $1,3 \%$ \\
\hline Granit & - & - & 4 & $3,9 \%$ & - & - & 4 & $1,3 \%$ \\
\hline Amphibolit & - & - & 1 & $1 \%$ & 1 & $0,5 \%$ & 2 & $0,7 \%$ \\
\hline Serpentinit & - & - & - & - & 2 & $1 \%$ & 2 & $0,7 \%$ \\
\hline Quarz-Sandstein & - & - & - & - & 2 & $1 \%$ & 2 & $0,7 \%$ \\
\hline Chalcedon & - & - & 1 & $1 \%$ & 1 & $0,5 \%$ & 2 & $0,7 \%$ \\
\hline Kalksandstein & - & - & - & - & 1 & $0,5 \%$ & 1 & $0,3 \%$ \\
\hline Gesamt & 1 & $100 \%$ & 102 & $100 \%$ & 204 & $100 \%$ & 307 & $100 \%$ \\
\hline
\end{tabular}

Tab. 2. Willendorf II: Rohmaterialhäufigkeit der geschlagenen Steinartefakte.

\begin{tabular}{|c|c|c|c|c|c|c|c|c|c|c|c|c|c|c|c|c|}
\hline \multirow[t]{2}{*}{ Grundformen } & \multicolumn{2}{|c|}{$\begin{array}{l}\text { Hornstein } \\
\text { alpin }\end{array}$} & \multicolumn{2}{|c|}{$\begin{array}{l}\text { Baltischer } \\
\text { Feuerstein }\end{array}$} & \multicolumn{2}{|c|}{ Radiolarit } & \multicolumn{2}{|c|}{ Granit } & \multicolumn{2}{|c|}{ Chalcedon } & \multicolumn{2}{|c|}{ Amphibolit } & \multicolumn{2}{|c|}{ Quarzit } & \multicolumn{2}{|c|}{ Gesamt } \\
\hline & $\mathrm{n}$ & $\%$ & $\mathrm{n}$ & $\%$ & $\mathrm{n}$ & $\%$ & $\mathrm{n}$ & $\%$ & $\mathrm{n}$ & $\%$ & $\mathrm{n}$ & $\%$ & $\mathrm{n}$ & $\%$ & $\mathrm{n}$ & $\%$ \\
\hline Klinge & 3 & $42,9 \%$ & 3 & $42,9 \%$ & 1 & $14,3 \%$ & - & - & - & - & - & - & - & - & 7 & $100 \%$ \\
\hline Lamelle & 26 & $66,7 \%$ & 13 & $33,3 \%$ & - & - & - & - & - & - & - & - & - & - & 39 & $100 \%$ \\
\hline Abschlag & 24 & $55,8 \%$ & 9 & $20,9 \%$ & 4 & $9,3 \%$ & 3 & $7 \%$ & 1 & $2,3 \%$ & 1 & $2,3 \%$ & 1 & $2,3 \%$ & 43 & $100 \%$ \\
\hline Trümmer & 1 & $50 \%$ & - & - & 1 & $50 \%$ & - & - & - & - & - & - & - & - & 2 & $100 \%$ \\
\hline $\begin{array}{l}\text { Stichelabfall } \\
\text { bzw. -lamelle }\end{array}$ & 1 & $100 \%$ & - & - & - & - & - & - & - & - & - & - & - & - & 1 & $100 \%$ \\
\hline Abspliss & 5 & $50 \%$ & 3 & $30 \%$ & 1 & $10 \%$ & 1 & $10 \%$ & - & - & - & - & - & - & 10 & $100 \%$ \\
\hline Gesamt & 60 & $58,8 \%$ & 28 & $27,5 \%$ & 7 & $6,9 \%$ & 4 & $3,9 \%$ & 1 & $1 \%$ & 1 & $1 \%$ & 1 & $1 \%$ & 102 & $100 \%$ \\
\hline
\end{tabular}

Tab. 3. Willendorf II, AH 6: Grundformenhäufigkeit pro Rohmaterial.

\begin{tabular}{|l|c|c|c|c|c|}
\hline Werkzeugtyp & Baltischer Feuerstein & Radiolarit & Quarzit & Hornstein alpin & Gesamt \\
\hline Stichel & 1 & - & - & 1 & 2 \\
\hline Kantenretusche & 1 & - & - & 1 & $\mathbf{2}$ \\
\hline Rückengestumpftes Element & 1 & - & - & 1 & $\mathbf{2}$ \\
\hline Kratzer & - & 1 & 1 & - & $\mathbf{2}$ \\
\hline Gesamt & $\mathbf{3}$ & 1 & 1 & $\mathbf{3}$ & $\mathbf{8}$ \\
\hline
\end{tabular}

Tab. 4. Willendorf II, AH 6: Werkzeugtypen pro Rohmaterial. 


\begin{tabular}{|l|c|c|c|c|c|c|c|c|c|c|}
\hline \multirow{2}{*}{ Rohmaterial } & \multicolumn{2}{|c|}{$\mathbf{0} \%$} & \multicolumn{2}{|c|}{$1-33 \%$} & \multicolumn{2}{|c|}{$33-66 \%$} & \multicolumn{2}{|c|}{$\mathbf{6 6 - 9 9 \%}$} & \multicolumn{2}{c|}{ Gesamt } \\
\hline & $\mathrm{n}$ & $\%$ & $\mathrm{n}$ & $\%$ & $\mathrm{n}$ & $\%$ & $\mathrm{n}$ & $\%$ & $\mathrm{n}$ & $\%$ \\
\hline Hornstein alpin & 50 & $83,3 \%$ & 5 & $8,3 \%$ & 4 & $6,7 \%$ & 1 & $1,7 \%$ & $\mathbf{6 0}$ & $100 \%$ \\
\hline Baltischer Feuerstein & 16 & $57,1 \%$ & 7 & $25 \%$ & 3 & $10,7 \%$ & 2 & $7,1 \%$ & $\mathbf{2 8}$ & $100 \%$ \\
\hline Radiolarit & 2 & $28,6 \%$ & 3 & $42,9 \%$ & 2 & $28,6 \%$ & - & - & 7 & $100 \%$ \\
\hline Granit & 1 & $25 \%$ & 2 & $50 \%$ & 1 & $25 \%$ & - & - & 4 & $100 \%$ \\
\hline Chalcedon & 1 & $100 \%$ & - & - & - & - & - & - & 1 & $100 \%$ \\
\hline Amphibolit & - & - & - & - & - & - & 1 & $100 \%$ & 1 & $100 \%$ \\
\hline Quarzit & 1 & $100 \%$ & - & - & - & - & - & - & 1 & $100 \%$ \\
\hline Gesamt & 71 & $\mathbf{6 9 , 6} \%$ & 17 & $16,7 \%$ & 10 & $\mathbf{9 , 8} \%$ & 4 & $3,9 \%$ & 102 & $100 \%$ \\
\hline
\end{tabular}

Tab. 5. Willendorf II, AH 6: Anteil der Bedeckung mit natürlichen Oberflächen pro Rohmaterial.

Das geringe Auftreten von thermischen Veränderungen (Tab. 6) an den verschiedenen Rohmaterialien lässt darauf schließen, dass es sich eher um zufälligen Kontakt mit Feuer als um kontrolliertes und systematisches Tempern, welches als intentionelle thermische Behandlung zur Verbesserung der Bearbeitungseigenschaften von Silices verstanden wird, ${ }^{134}$ handelt. Lediglich bei Radiolarit ist der Anteil an Stücken mit thermischer Einwirkung höher als bei den anderen Rohmaterialien, jedoch ist die Stückzahl von Radiolarit sehr gering.

\subsubsection{Rohmaterialien der Steinartefakte des $\mathrm{AH} 8$}

Im AH 8 dominiert die Gruppe der Quarzite mit 41,2 \% (Tab. 2). Danach folgen Kieselkalke mit $24 \%$ und Jaspise mit 16,7 \%. Auffällig ist, dass kein Vertreter der Gruppe der alpinen Hornsteine nachgewiesen wurde. Die Rohmaterialien stammen vorwiegend aus lokalen Lagerstätten. Der Anteil an Feuerstein liegt bei lediglich 3,4 \%.

Bei den Kernen dominieren die drei größten Rohmaterialgruppen (Tab. 7). Abschläge aus allen Rohmaterialien sind vorhanden (Tab. 8). Bei der Gewinnung von vor allem Klingen, aber auch Lamellen, spielten Quarzit, Kieselkalk, Jaspis, Radiolarit und Feuerstein eine Rolle, doch weiterführende Schlussfolgerungen wollen wir auf Grund der geringen Stückzahl vermeiden.

Bei den Werkzeugtypen sind die fünf größten Rohmaterialgruppen vertreten (Tab. 9). Das am häufigsten vorkommende Rohmaterial Quarzit macht mehr als die Hälfte des Werkzeugbestands aus. Zur Werkzeugherstellung dienten bei allen fünf Rohmaterialien in erster Linie Klingen und Abschläge.

134 Weiner 1985. - Domanski, Webb 1992. - Domanski, Webb, BOLAND 1994.
Der Anteil an natürlichen Oberflächen der dominierenden Rohmaterialgruppen, Quarzit, Kieselkalk, Jaspis und Radiolarit, reicht von Grundformen ohne natürliche Oberflächen auf der Dorsalfläche bis zu solchen mit kompletter dorsaler Bedeckung (Tab. 10). Daher kann davon ausgegangen werden, dass diese Rohmaterialien zumindest zum Teil in Form von Knollen vor der Entrindung in die Fundstelle eingebracht wurden, um dann vor Ort verarbeitet zu werden. Beim baltischen Feuerstein wurde bei 85,7 \% keine natürliche Oberfläche dorsal festgestellt und beim Rest war maximal ein Drittel der Dorsalfläche bedeckt. Trotz der geringen Stückzahl kann davon ausgegangen werden, dass dieses nicht-lokale Rohmaterial bereits entrindet zur Fundstelle transportiert wurde.

Thermische Veränderungen an den Artefakten wurden selten festgestellt (Tab. 11). Daher wird davon ausgegangen, dass kein systematisches Tempern der Rohmaterialien erfolgte. Der Anteil an hitzebedingten Einwirkungen bleibt bei allen Rohmaterialen unter $10 \%$, nur Jaspis bildet mit mehr als $20 \%$ eine Ausnahme. Zu den sieben Stücken aus Jaspis mit thermischen Veränderungen zählen zwei Hitzetrümmer, ein Kern, eine Kernscheibe sowie drei Abschläge.

\subsection{Ergebnisse zur Steintechnologie in AH 6}

\subsubsection{Grundformen}

\subsubsection{Grundformenanteile}

Die Abschläge stellen mit 40,2 \% die größte Artefaktklasse dar, doch liegt der Anteil der Lamellen auch bei über einem Drittel (Tab. 12). Absplisse sind mit 9,8 \% stärker vertreten als Klingen, die nur 3,9 \% des Inventars ausmachen. Das Fehlen von größeren Produkten könnte darauf zurückgeführt werden, dass die Grundformproduktion auf eher 

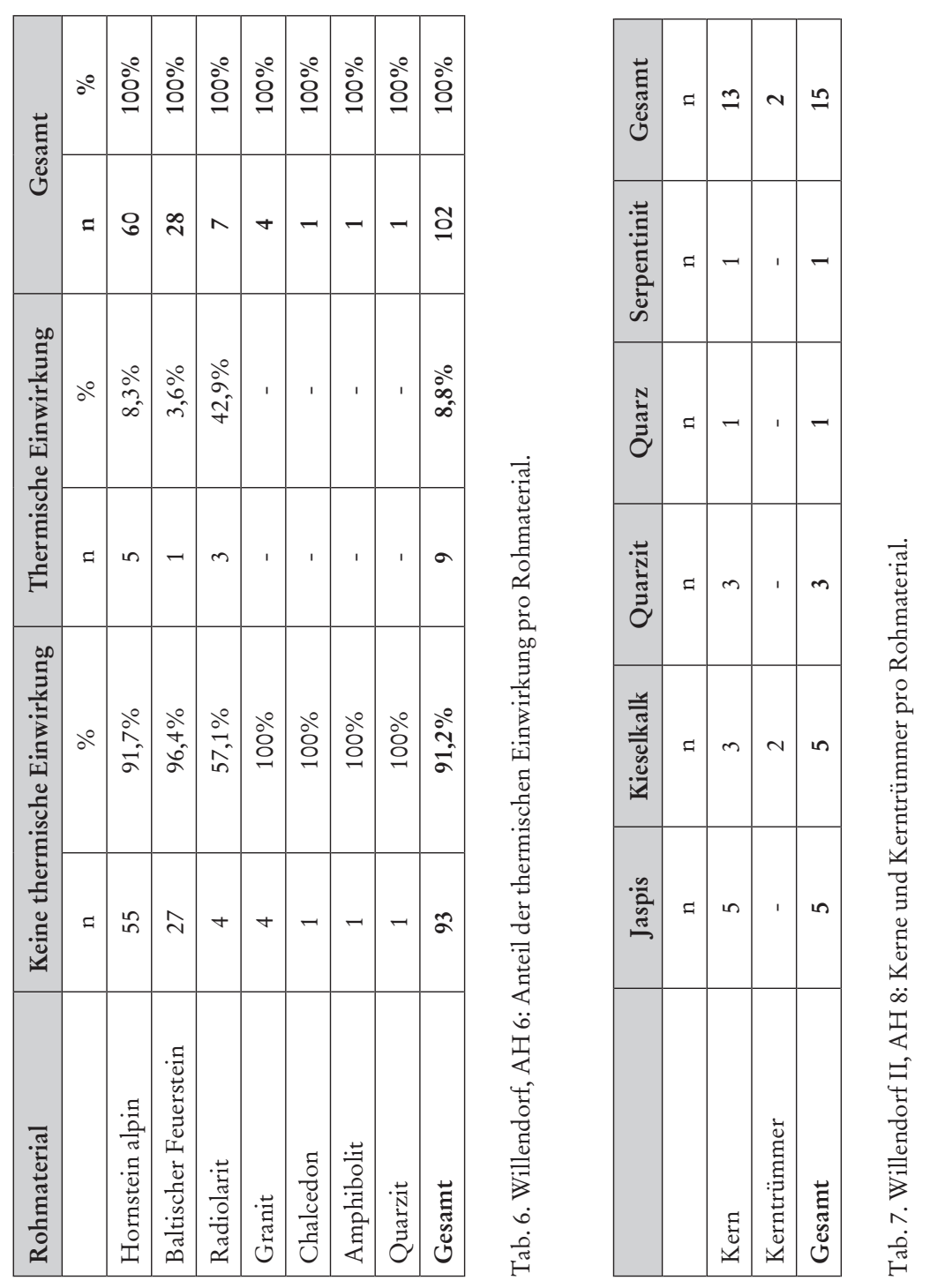

\begin{tabular}{|c|c|c|c|c|c|c|c|}
\hline \multirow{2}{*}{$\begin{array}{l}\breve{\Xi} \\
\tilde{\Xi} \\
\tilde{J} \\
心\end{array}$} & $0^{\circ}$ & $\stackrel{\circ}{\stackrel{0}{0}}$ & $\stackrel{\circ}{8}$ & $\stackrel{\circ}{8}$ & 음 & $\stackrel{\circ}{\circ}$ & $\stackrel{\circ}{\circ}$ \\
\hline & $=$ & $q$ & $\simeq$ & 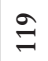 & $a$ & 6 & $\stackrel{\infty}{\sim}$ \\
\hline \multirow{2}{*}{ 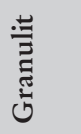 } & $\delta^{\circ}$ & I & ' & $\stackrel{\stackrel{\circ}{+}}{\stackrel{m}{m}}$ & & & $\stackrel{\circ}{\stackrel{2}{i}}$ \\
\hline & 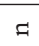 & I & 1 & + & 1 & & $\theta$ \\
\hline \multirow{2}{*}{ 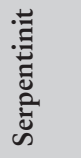 } & $\delta^{\circ}$ & ' & ' & $\begin{array}{l}\stackrel{0}{0} \\
\infty \\
0 \\
0\end{array}$ & ' & & in \\
\hline & 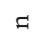 & ' & ' & - & 1 & 1 & - \\
\hline \multirow{2}{*}{ 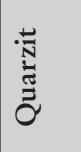 } & $\partial^{\circ}$ & $\begin{array}{l}\stackrel{0}{0} \\
i n \\
\tilde{n}^{2}\end{array}$ & $\begin{array}{l}\stackrel{0}{\hat{N}} \\
\hat{\sigma}\end{array}$ & $\begin{array}{c}\stackrel{0}{\circ} \\
\dot{+} \\
\stackrel{f}{f}\end{array}$ & $\begin{array}{l}\stackrel{0}{\circ} \\
\dot{+} \\
\dot{f}\end{array}$ & $\begin{array}{l}\stackrel{0}{\hat{n}} \\
\text { ŝ́ }\end{array}$ & $\begin{array}{l}\stackrel{0}{\circ} \\
\stackrel{a}{q}\end{array}$ \\
\hline & $\Rightarrow$ & $\approx$ & $N$ & in & + & + & $\bar{\infty}$ \\
\hline \multirow{2}{*}{ 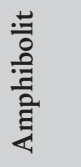 } & $\partial^{\circ}$ & 1 & ' & $\begin{array}{l}\stackrel{0}{0} \\
\infty \\
0\end{array}$ & I & & 号 \\
\hline & $F$ & 1 & I & - & 1 & 1 & - \\
\hline \multirow{2}{*}{ 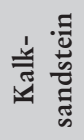 } & $\alpha^{\circ}$ & ' & I & $\begin{array}{l}\stackrel{0}{0} \\
\infty \\
0\end{array}$ & 1 & I & in \\
\hline & $\Rightarrow$ & 1 & ' & - & ' & , & - \\
\hline \multirow{2}{*}{ 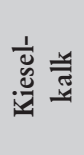 } & $\partial^{\circ}$ & 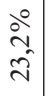 & $\frac{\stackrel{\circ}{\hat{N}}}{\vec{f}}$ & $\begin{array}{l}\stackrel{0}{0} \\
\hat{i} \\
\hat{v}\end{array}$ & $\stackrel{\circ}{=}$ & & $\stackrel{0}{\tilde{m}^{2}}$ \\
\hline & 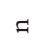 & 으 & in & $\stackrel{\infty}{\sim}$ & - & 1 & J \\
\hline \multirow{2}{*}{ 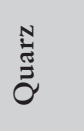 } & $0^{\circ}$ & 1 & ' & $\begin{array}{l}\stackrel{\circ}{+} \\
m\end{array}$ & ' & & $\frac{0}{i}$ \\
\hline & $\Rightarrow$ & 1 & ' & + & 1 & 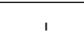 & + \\
\hline \multirow{2}{*}{$\begin{array}{l}\frac{\Xi}{0} \\
\frac{\tilde{U}}{\tilde{U}} \\
\frac{\mathrm{U}}{\mathrm{J}} \\
\end{array}$} & $d^{\circ}$ & ' & ' & $\begin{array}{l}\stackrel{0}{0} \\
\infty_{0}^{\circ}\end{array}$ & ' & & iे \\
\hline & $=$ & ' & I & - & I & 1 & - \\
\hline \multirow[t]{2}{*}{ 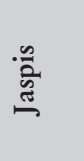 } & $\partial^{\circ}$ & $\begin{array}{l}\stackrel{0}{0} \\
\text { Na } \\
\hat{\sim}\end{array}$ & iे & ڤू & $\stackrel{\circ}{m^{2}}$ & $\stackrel{\stackrel{े}{m}}{\hat{m}^{2}}$ & $\stackrel{\circ}{\stackrel{2}{2}}$ \\
\hline & $\Rightarrow$ & 으 & $m$ & $=$ & $m$ & N & নे \\
\hline \multirow{2}{*}{ 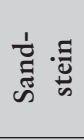 } & $\alpha^{\circ}$ & ' & ' & $\stackrel{\circ}{\stackrel{\circ}{=}}$ & ' & 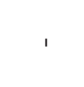 & $\stackrel{\circ}{\circ}$ \\
\hline & $\Rightarrow$ & 1 & I & $N$ & 1 & 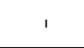 & $N$ \\
\hline \multirow{2}{*}{ 苞 } & $0^{\circ}$ & $\stackrel{\circ}{\wedge}$ & 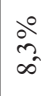 & $\begin{array}{l}0 \\
0 \\
0 \\
n^{\prime}\end{array}$ & $\begin{array}{l}\stackrel{\circ}{\circ} \\
\Rightarrow\end{array}$ & & $\frac{\circ}{\stackrel{\circ}{\alpha}}$ \\
\hline & $F$ & $m$ & - & $a$ & - & I & \pm \\
\hline \multirow{2}{*}{ 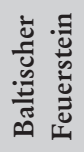 } & $\alpha^{\circ}$ & $\stackrel{\circ}{\wedge}$ & $\begin{array}{c}\stackrel{0}{2} \\
\hat{m}^{2}\end{array}$ & $\begin{array}{l}\text { o } \\
\text { in } \\
\text { in }\end{array}$ & 1 & ' & $\stackrel{\circ}{\stackrel{\circ}{\circ}}$ \\
\hline & $\Rightarrow$ & $n$ & - & $m$ & I & & $n$ \\
\hline 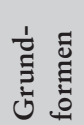 & & 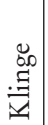 & 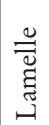 & $\begin{array}{l}0 \\
\frac{\pi}{3} \\
\frac{1}{0} \\
0 \\
\frac{0}{4}\end{array}$ & 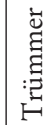 & 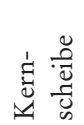 & $\begin{array}{l}\vec{E} \\
\tilde{ت} \\
\tilde{U} \\
\mathcal{U}\end{array}$ \\
\hline
\end{tabular}




\begin{tabular}{|c|c|c|c|c|c|c|c|c|c|c|c|c|}
\hline \multirow[t]{2}{*}{ Werkzeugtyp } & \multicolumn{2}{|c|}{ Baltischer Feuerstein } & \multicolumn{2}{|c|}{ Radiolarit } & \multicolumn{2}{|c|}{ Quarzit } & \multicolumn{2}{|c|}{ Kieselkalk } & \multicolumn{2}{|c|}{ Jaspis } & \multicolumn{2}{|c|}{ Gesamt } \\
\hline & $\mathrm{n}$ & $\%$ & $\mathrm{n}$ & $\%$ & $\mathrm{n}$ & $\%$ & $\mathrm{n}$ & $\%$ & $\mathrm{n}$ & $\%$ & $\mathbf{n}$ & $\%$ \\
\hline Ausgesplittertes Stück & 1 & $5,9 \%$ & - & - & 10 & $58,8 \%$ & 2 & $11,8 \%$ & 4 & $23,5 \%$ & 17 & $100 \%$ \\
\hline Kantenretusche & - & - & 1 & $11,1 \%$ & 4 & $44,4 \%$ & 1 & $11,1 \%$ & 3 & $33,3 \%$ & 9 & $100 \%$ \\
\hline Stichel & - & - & - & - & 3 & $100 \%$ & - & - & - & - & 3 & $100 \%$ \\
\hline Kratzer mit Kantenretusche & - & - & - & - & - & - & 1 & $50 \%$ & 1 & $50 \%$ & 2 & $100 \%$ \\
\hline Spitzklinge & - & - & - & - & 1 & $50 \%$ & - & - & 1 & $50 \%$ & 2 & $100 \%$ \\
\hline $\begin{array}{l}\text { Ausgesplittertes Stück mit } \\
\text { Kantenretusche }\end{array}$ & - & - & - & - & 2 & $100 \%$ & - & - & - & - & 2 & $100 \%$ \\
\hline Endretusche & - & - & 1 & $50 \%$ & - & - & - & - & 1 & $50 \%$ & 2 & $100 \%$ \\
\hline Kratzer & - & - & - & - & 1 & $100 \%$ & - & - & - & - & 1 & $100 \%$ \\
\hline Bohrer mit Kantenretusche & 1 & $100 \%$ & - & - & - & - & - & - & - & - & 1 & $100 \%$ \\
\hline Gezähntes Stück & - & - & - & - & 1 & $100 \%$ & - & - & - & - & 1 & $100 \%$ \\
\hline Gesamt & 2 & $5 \%$ & 2 & $5 \%$ & 22 & $55 \%$ & 4 & $10 \%$ & 10 & $25 \%$ & 40 & $100 \%$ \\
\hline
\end{tabular}

Tab. 9. Willendorf II, AH 8: Werkzeugtypen pro Rohmaterial.

\begin{tabular}{|c|c|c|c|c|c|c|c|c|c|c|c|c|}
\hline \multirow[t]{2}{*}{ Rohmaterial } & \multicolumn{2}{|c|}{$0 \%$} & \multicolumn{2}{|c|}{$1-33 \%$} & \multicolumn{2}{|c|}{$33-66 \%$} & \multicolumn{2}{|c|}{$66-99 \%$} & \multicolumn{2}{|c|}{$100 \%$} & \multicolumn{2}{|c|}{ Gesamt } \\
\hline & $\mathrm{n}$ & $\%$ & $\mathrm{n}$ & $\%$ & $\mathrm{n}$ & $\%$ & $\mathrm{n}$ & $\%$ & $\mathrm{n}$ & $\%$ & $\mathrm{n}$ & $\%$ \\
\hline Quarzit & 51 & $63 \%$ & 16 & $19,8 \%$ & 8 & $9,9 \%$ & 5 & $6,2 \%$ & 1 & $1,2 \%$ & 81 & $100 \%$ \\
\hline Kieselkalk & 15 & $34,1 \%$ & 15 & $34,1 \%$ & 6 & $13,6 \%$ & 3 & $6,8 \%$ & 5 & $11,4 \%$ & 44 & $100 \%$ \\
\hline Jaspis & 13 & $44,8 \%$ & 13 & $44,8 \%$ & 1 & $3,4 \%$ & 1 & $3,4 \%$ & 1 & $3,4 \%$ & 29 & $100 \%$ \\
\hline Radiolarit & 4 & $28,6 \%$ & 2 & $14,3 \%$ & 4 & $28,6 \%$ & 2 & $14,3 \%$ & 2 & $14,3 \%$ & 14 & $100 \%$ \\
\hline Baltischer Feuerstein & 6 & $85,7 \%$ & 1 & $14,3 \%$ & - & - & - & - & - & - & 7 & $100 \%$ \\
\hline Quarz & - & - & 1 & $25 \%$ & 3 & $75 \%$ & - & - & - & - & 4 & $100 \%$ \\
\hline Granulit & 1 & $25 \%$ & 1 & $25 \%$ & 1 & $25 \%$ & 1 & $25 \%$ & - & - & 4 & $100 \%$ \\
\hline Quarz-Sandstein & - & - & - & - & 1 & $50 \%$ & 1 & $50 \%$ & - & - & 2 & $100 \%$ \\
\hline Chalcedon & 1 & $100 \%$ & - & - & - & - & - & - & - & - & 1 & $100 \%$ \\
\hline Serpentinit & - & - & 1 & $100 \%$ & - & - & - & - & - & - & 1 & $100 \%$ \\
\hline Kalksandstein & 1 & $100 \%$ & - & - & - & - & - & - & - & - & 1 & $100 \%$ \\
\hline Amphibolit & 1 & $100 \%$ & - & - & - & - & - & - & - & - & 1 & $100 \%$ \\
\hline Gesamt & 93 & $49,2 \%$ & 50 & $26,5 \%$ & 24 & $12,7 \%$ & 13 & $6,9 \%$ & 9 & $4,8 \%$ & 189 & $100 \%$ \\
\hline
\end{tabular}

Tab. 10. Willendorf II, AH 8: Anteil der Bedeckung mit natürlichen Oberflächen pro Rohmaterial. 


\begin{tabular}{|l|c|c|c|c|c|c|}
\hline Rohmaterial & Keine thermische Einwirkung & Thermische Einwirkung & \multicolumn{2}{|c|}{ Gesamt } \\
\hline & $\mathrm{n}$ & $\%$ & $\mathrm{n}$ & $\%$ & $\mathrm{n}$ & $\%$ \\
\hline Quarzit & 79 & $94 \%$ & 5 & $6 \%$ & 84 & $100 \%$ \\
\hline Kieselkalk & 48 & $98 \%$ & 1 & $2 \%$ & 49 & $100 \%$ \\
\hline Jaspis & 27 & $79,4 \%$ & 7 & $20,6 \%$ & 34 & $100 \%$ \\
\hline Radiolarit & 13 & $92,9 \%$ & 1 & $7,1 \%$ & 14 & $100 \%$ \\
\hline Baltischer Feuerstein & 7 & $100 \%$ & - & - & 7 & $100 \%$ \\
\hline Quarz & 5 & $100 \%$ & - & - & 5 & $100 \%$ \\
\hline Granulit & 4 & $100 \%$ & - & - & 4 & $100 \%$ \\
\hline Quarz-Sandstein & 2 & $100 \%$ & - & - & 2 & $100 \%$ \\
\hline Serpentinit & 2 & $100 \%$ & - & - & 2 & $100 \%$ \\
\hline Kalksandstein & 1 & $100 \%$ & - & - & 1 & $100 \%$ \\
\hline Chalcedon & 1 & $100 \%$ & - & - & 1 & $100 \%$ \\
\hline Amphibolit & 1 & $100 \%$ & - & - & 1 & $100 \%$ \\
\hline Gesamt & 190 & $93,1 \%$ & 14 & $\mathbf{6 , 9} \%$ & 204 & $100 \%$ \\
\hline
\end{tabular}

Tab. 11. Willendorf II, AH 8: Anteil der thermischen Einwirkung pro Rohmaterial.

\begin{tabular}{|c|c|c|c|c|c|c|c|c|}
\hline \multirow[t]{2}{*}{ Artefaktklasse } & \multicolumn{2}{|c|}{ AH 4} & \multicolumn{2}{|c|}{ AH 6} & \multicolumn{2}{|c|}{ AH 8} & \multicolumn{2}{|c|}{ Modern } \\
\hline & $\mathrm{n}$ & $\%$ & $\mathrm{n}$ & $\%$ & $\mathrm{n}$ & $\%$ & $\mathrm{n}$ & $\%$ \\
\hline Klinge & - & - & 4 & $3,9 \%$ & 39 & $15,4 \%$ & - & - \\
\hline Primäre Kernkantenklinge, zweiseitig & - & - & 1 & $1 \%$ & 2 & $0,8 \%$ & - & - \\
\hline Primäre Kernkantenklinge, einseitig & - & - & 2 & $2 \%$ & 1 & $0,4 \%$ & - & - \\
\hline Sekundäre Kernkantenklinge & - & - & - & - & 1 & $0,4 \%$ & - & - \\
\hline Lamelle & 1 & $100 \%$ & 37 & $36,3 \%$ & 9 & $3,6 \%$ & - & - \\
\hline Primäre Kernkantenlamelle, zweiseitig & - & - & 1 & $1 \%$ & - & - & - & - \\
\hline Sekundäre Kernkantenlamelle & - & - & 1 & $1 \%$ & 3 & $1,2 \%$ & - & - \\
\hline Abschlag & - & - & 41 & $40,2 \%$ & 107 & $42,3 \%$ & - & - \\
\hline Primärer Kernkantenabschlag, einseitig & - & - & 1 & $1 \%$ & 2 & $0,8 \%$ & - & - \\
\hline Sekundärer Kernkantenabschlag & - & - & 1 & $1 \%$ & 1 & $0,4 \%$ & - & - \\
\hline Stichelabfall bzw. -lamelle & - & - & 1 & $1 \%$ & - & - & - & - \\
\hline Abfall von Ausgesplittertem Stück & - & - & - & - & 9 & $3,6 \%$ & - & - \\
\hline Abspliss & - & - & 10 & $9,8 \%$ & - & - & - & - \\
\hline Kern & - & - & - & - & 13 & $5,1 \%$ & - & - \\
\hline Kerntrümmer & - & - & - & - & 2 & $0,8 \%$ & - & - \\
\hline Schlagstein & - & - & - & - & 4 & $1,6 \%$ & - & - \\
\hline Manuport & - & - & - & - & 45 & $17,8 \%$ & 3 & $100 \%$ \\
\hline Kernscheibe & - & - & - & - & 6 & $2,3 \%$ & - & - \\
\hline Trümmer & - & - & 2 & $2 \%$ & 9 & $3,6 \%$ & - & - \\
\hline Gesamt & 1 & $100 \%$ & 102 & $100 \%$ & 253 & $100 \%$ & 3 & $100 \%$ \\
\hline
\end{tabular}

Tab. 12. Willendorf II: Artefaktklassenhäufigkeiten. 
kleinere Formen, im Speziellen Lamellen, abzielte. Kernkantengrundformen bilden zwar einen geringen Anteil des Gesamtmaterials (Tab. 12), aber durch ihr Auftreten wird die Anlage einer Kernkante als Leitgrat für das Lösen einer Grundform belegt. Es ist eine Stichellamelle vorhanden und dies deutet darauf hin, dass auch Stichel als Kerne für die Lamellengewinnung eingesetzt wurden, jedoch fallen die Stichel des Inventars nicht in diese Kategorie. Trümmer, Abfallprodukte im Herstellungsprozess, sind mit zwei Stücken vertreten (Tab. 12).

\subsubsection{Maße}

Bei der Längenverteilung wird deutlich, dass der Großteil der in der Länge vollständig erhaltenen Steinartefakte sich in einem Spektrum zwischen 10 und $30 \mathrm{~mm}$ bewegt (Tab. 13). Lediglich ein Abschlag weist eine Länge von mehr als $40 \mathrm{~mm}$ auf. Vier Artefakte liegen unter $10 \mathrm{~mm}$. Trotz der geringen Anzahl an in der Länge vollständigen Grundformen zeichnet sich die Kleinheit des Inventars ab, wobei keine einzige Klinge in der Länge vollständig erhalten ist. Bei der Längenverteilung nach Grundformen (Tab. 14) zeigt sich, dass die Variationsbreite bei den Abschlägen und Absplissen wesentlich größer ist als bei den Lamellen, womit zumindest tendenziell eine Standardisierung der Lamellen angenommen werden kann. Die Längen der Lamellen liegen allesamt in einem Bereich zwischen 15 und $29 \mathrm{~mm}$. Die Standardabweichung bei den Lamellen liegt bei 3,6 mm, bei den Abschlägen und Absplissen hingegen bei 8,7 mm.

Die Breitenverteilung (Tab. 15) zeigt, dass 55 der Grundformen und damit $87 \%$ der in der Breite vollständig erhaltenen Grundformen in einem Bereich zwischen 2,8 und 17,5 mm liegen. Außerdem sind $97 \%$ der Grundformen unter $30 \mathrm{~mm}$ breit. Lediglich zwei Abschläge überschreiten diesen Wert. Wenn man die Breitenverteilung aufgeschlüsselt nach Abschlägen und Absplissen bzw. Klingen und Lamellen (Tab. 16) betrachtet, zeigt sich, dass die Abschläge und Absplisse eine größere Streuung von 5,6 bis 32,7 mm aufweisen und breiter ausfallen als die Klingen und Lamellen mit 2,8 bis $16,6 \mathrm{~mm}$.

Bei der Dickenverteilung (Tab. 17) wurde ermittelt, dass die Grundformen eine Dicke von 0,5 bis 16,3 mm aufweisen. 94,1 \% der Grundformen jedoch liegen im Bereich von 0,5 bis 6,8 mm. Die beiden Abschläge, die bereits die höchsten Werte in Bezug auf die Breite ergaben, lieferten auch bei der Dicke die Maximalwerte mit jeweils über $10 \mathrm{~mm}$. Nach Grundformen aufgegliedert zeigt sich, dass die Abschläge und Absplisse mit 0,6 bis 16,3 mm wiederum eine größere Spanne als die Klingen und Lamellen mit 0,5 bis $8 \mathrm{~mm}$ aufweisen (Tab. 18). Außerdem wurde bei den Klingen und
Lamellen festgestellt, dass 56,4\% zwischen 0,5 und $2 \mathrm{~mm}$ dick sind und danach die Anzahl rapide bis $8 \mathrm{~mm}$ abfällt.

\subsubsection{Dorsale Bedeckung mit natürlichen Oberflächen}

Im gesamten Inventar des AH 6 ist, wie oben bereits angesprochen, keine Grundform dorsal komplett mit natürlichen Oberflächen bedeckt. Bei Klingen und Lamellen dominieren diejenigen Stücke, die gar keine natürlichen Oberflächen aufweisen (Tab. 19). Auch bei den Abschlägen wurden mit 58,1\% hauptsächlich solche ohne dorsale Bedeckung mit natürlichen Oberflächen nachgewiesen. Es liegen aber trotzdem bei diesem Grundformtyp die meisten Stücke mit Resten natürlicher Oberflächen vor, die aus den Anfangsstadien des Kernabbaus stammen und der Präparation gedient haben dürften. Die Lage der natürlichen Oberflächen beschränkt sich bei allen Grundformen fast ausschließlich auf den distalen und dextrolateralen Bereich. Dieses Muster an vorwiegend dextrolateralen und distalen Enden mit natürlichen Oberflächen könnte auf eine bestimmte Gestaltung des Kernabbaus, vor allem in Bezug auf die Lamellen, hinweisen (siehe Abschnitt 8.1).

\subsubsection{Schlagflächenreste}

Anhand des Verhältnisses der Schlagflächenrest-Breite zur Schlagflächenrest-Dicke ergab sich, dass die Grundformen zwar insgesamt relativ schmale und kleine Schlagflächenreste besitzen, dass die Schlagflächenreste der Abschläge und Absplisse aber eindeutig breiter und dicker (Tab. 20, 21) sind als jene der Klingen und Lamellen (Abb. 7).

Die Art des Schlagflächenrestes liefert einen Hinweis, ob eine mehr oder weniger aufwändige Präparation der Schlagfläche des Kerns während des Kernabbaus stattfand. ${ }^{135}$ Der Aufwand dürfte eher gering ausgefallen sein, da im Inventar bei der Art des Schlagflächenrestes glatte Schlagflächenreste dominieren (Tab. 22). Bei den Lamellen liegen glatte und lineare Schlagflächenreste vor, bei den Klingen ausschließlich glatte. Auch bei den Abschlägen finden sich hauptsächlich Stücke mit glattem Schlagflächenrest, allerdings gibt es auch einige mit Schlagflächenrest bedeckt mit natürlichen Oberflächen.

Bei der Form des Schlagflächenrestes zeigt sich, dass ein Drittel der Grundformen eine lineare Form aufweist (Tab. 23). Bei den Lamellen dominiert der lineare Schlagflächenrest. Es wurde keine einzige Lamelle mit einem unregelmäßigen Schlagflächenrest nachgewiesen. Bei den Abschlägen hingegen sind jene mit ovalem bzw. geflügeltem Schlagflächenrest am häufigsten vertreten, gefolgt von jenen mit unregelmäßiger Form des Schlagflächenrestes.

135 Moreau 2009, 105 


\begin{tabular}{|l|c|}
\hline AH 6 & Grundformen \\
\hline Anzahl & 31 \\
\hline Min & 6,84 \\
\hline Max & 43,23 \\
\hline Mittelwert & 18,77 \\
\hline Stand. Abw. & 7,25 \\
\hline Median & 19,8 \\
\hline
\end{tabular}

Tab. 13. Willendorf II, AH 6: Deskriptive Statistik der Länge (in mm) aller in der Länge vollständig erhaltenen Grundformen.

\begin{tabular}{|l|c|}
\hline AH 6 & Grundformen \\
\hline Anzahl & 62 \\
\hline Min & 2,8 \\
\hline Max & 32,68 \\
\hline Mittelwert & 12,15 \\
\hline Stand. Abw. & 7,01 \\
\hline Median & 10,51 \\
\hline
\end{tabular}

Tab. 15. Willendorf II, AH 6: Deskriptive Statistik der Breite (in $\mathrm{mm}$ ) aller in der Breite vollständig erhaltenen Grundformen.

\begin{tabular}{|l|c|}
\hline AH 6 & Grundformen \\
\hline Anzahl & 85 \\
\hline Min & 0,5 \\
\hline Max & 16,31 \\
\hline Mittelwert & 3,35 \\
\hline Stand. Abw. & 2,61 \\
\hline Median & 2,67 \\
\hline
\end{tabular}

Tab. 17. Willendorf II, AH 6: Deskriptive Statistik der Dicke (in mm) aller in der Dicke vollständig erhaltenen Grundformen.

\begin{tabular}{|l|c|c|}
\hline AH 6 & Lamellen/Klingen & Absplisse/Abschläge \\
\hline Anzahl & 14 & 17 \\
\hline Min & 15,49 & 6,84 \\
\hline Max & 29 & 43,23 \\
\hline Mittelwert & 21,6 & 16,45 \\
\hline Stand. Abw. & 3,63 & 8,67 \\
\hline Median & 21,7 & 15,2 \\
\hline
\end{tabular}

Tab. 14. Willendorf II, AH 6: Deskriptive Statistik der Länge (in $\mathrm{mm}$ ) aller in der Länge vollständig erhaltenen Lamellen bzw. Klingen sowie Absplisse bzw. Abschläge.

\begin{tabular}{|l|c|c|}
\hline AH 6 & Lamellen/Klingen & Absplisse/Abschläge \\
\hline Anzahl & 34 & 28 \\
\hline Min & 2,8 & 5,58 \\
\hline Max & 16,6 & 32,68 \\
\hline Mittelwert & 8,23 & 16,92 \\
\hline Stand. Abw. & 3,41 & 7,34 \\
\hline Median & 7,7 & 15,06 \\
\hline
\end{tabular}

Tab. 16. Willendorf II, AH 6: Deskriptive Statistik der Breite (in $\mathrm{mm}$ ) aller in der Breite vollständig erhaltenen Lamellen bzw. Klingen sowie Absplisse bzw. Abschläge.

\begin{tabular}{|l|c|c|}
\hline AH 6 & Lamellen/Klingen & Absplisse/Abschläge \\
\hline Anzahl & 39 & 46 \\
\hline Min & 0,5 & 0,6 \\
\hline Max & 8,03 & 16,31 \\
\hline Mittelwert & 2,4 & 4,16 \\
\hline Stand. Abw. & 1,77 & 2,94 \\
\hline Median & 1,82 & 3,53 \\
\hline
\end{tabular}

Tab. 18. Willendorf II, AH 6: Deskriptive Statistik der Dicke (in $\mathrm{mm}$ ) aller in der Dicke vollständig erhaltenen Lamellen bzw. Klingen sowie Absplisse bzw. Abschläge.

\begin{tabular}{|l|c|c|c|c|c|c|c|c|c|c|}
\hline \multirow{2}{*}{ Grundformen } & \multicolumn{2}{|c|}{ \% } & \multicolumn{2}{c|}{$1-33 \%$} & \multicolumn{2}{c|}{$33-\mathbf{6 6} \%$} & \multicolumn{2}{|c|}{$\mathbf{6 6 - 9 9} \%$} & \multicolumn{2}{c|}{ Gesamt } \\
\hline & $\mathrm{n}$ & $\%$ & $\mathrm{n}$ & $\%$ & $\mathrm{n}$ & $\%$ & $\mathrm{n}$ & $\%$ & $\mathrm{n}$ & $\%$ \\
\hline Klinge & 6 & $85,7 \%$ & 1 & $14,3 \%$ & - & - & - & - & 7 & $100 \%$ \\
\hline Lamelle & 32 & $80 \%$ & 5 & $12,5 \%$ & 2 & $5 \%$ & 1 & $2,5 \%$ & 40 & $100 \%$ \\
\hline Abschlag & 25 & $58,1 \%$ & 10 & $23,3 \%$ & 5 & $11,6 \%$ & 3 & $7 \%$ & 43 & $100 \%$ \\
\hline Abspliss & 7 & $70 \%$ & 1 & $10 \%$ & 2 & $20 \%$ & - & - & 10 & $100 \%$ \\
\hline Gesamt & 70 & $70 \%$ & 17 & $17 \%$ & 9 & $\mathbf{9} \%$ & 4 & $\mathbf{4} \%$ & 100 & $100 \%$ \\
\hline
\end{tabular}

Tab. 19. Willendorf II, AH 6: Anteile an natürlichen Oberflächen bei allen Grundformen. 


\section{- Abschläge \& Absplisse $\Delta$ Klingen \& Lamellen}

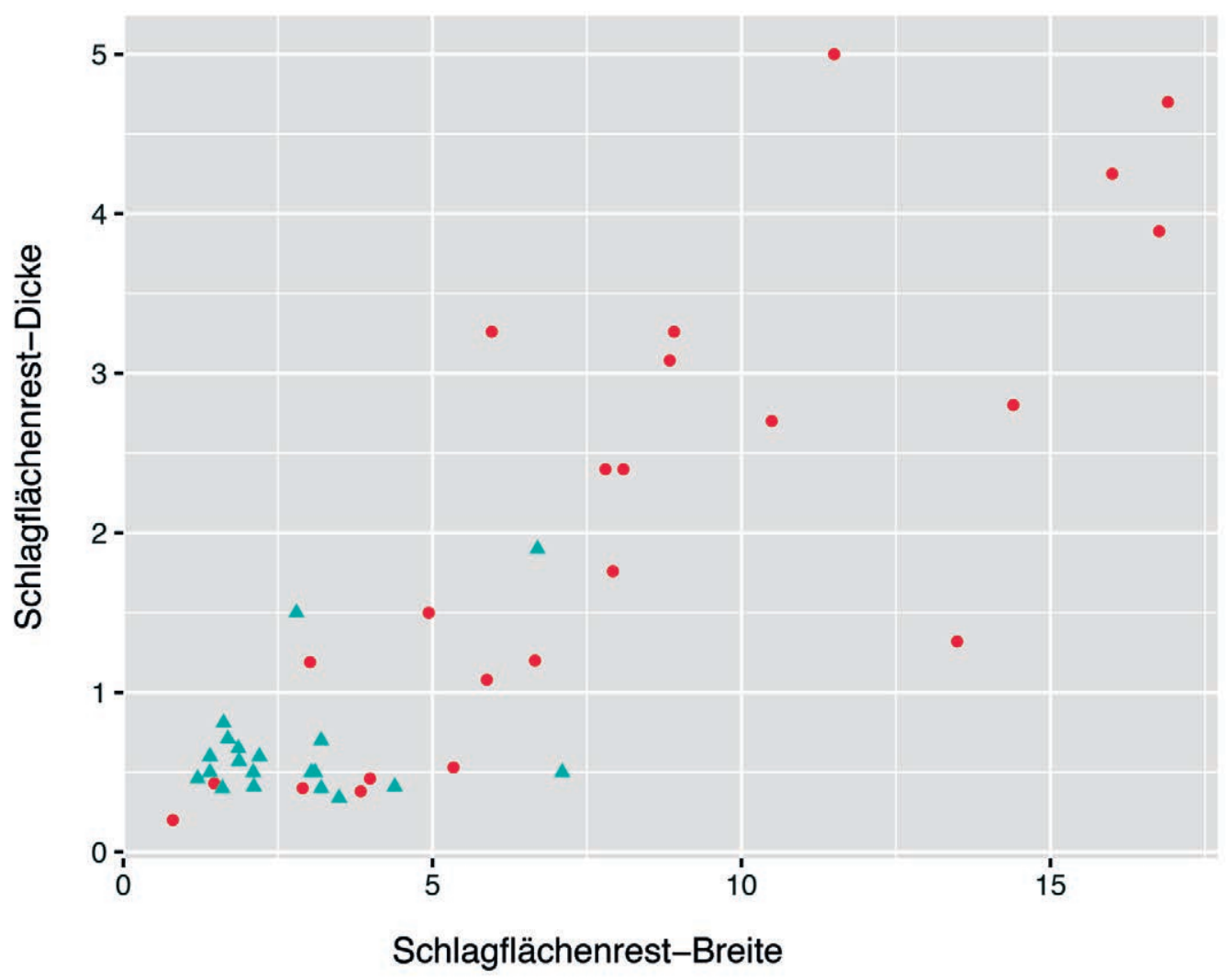

Abb. 7. Willendorf II, AH 6: Scatterplot der Breite und Dicke der Schlagflächenreste aller in der Länge vollständig oder proximal erhaltenen Abschläge und Absplisse sowie Lamellen und Klingen. Alle Angaben in Millimeter.

\begin{tabular}{|l|c|c|}
\hline AH 6 & Lamellen/Klingen & Absplisse/Abschläge \\
\hline Anzahl & 21 & 23 \\
\hline Mittelwert & 2,77 & 8,08 \\
\hline Stand. Abw. & 0,73 & 2,10 \\
\hline Varianz & 2,56 & 23,48 \\
\hline $\mathrm{F}=$ & \multicolumn{2}{|c|}{9,1712} \\
\hline $\mathrm{t}=$ & $\mathbf{2}$ \\
\hline$p=$ & \multicolumn{2}{|c|}{0,00025} \\
\hline
\end{tabular}

\begin{tabular}{|l|c|c|}
\hline AH 6 & Lamellen/Klingen & Absplisse/Abschläge \\
\hline Anzahl & 21 & 23 \\
\hline Mittelwert & 0,65 & 2,10 \\
\hline Stand. Abw. & 0,17 & 0,64 \\
\hline Varianz & 0,14 & 2,22 \\
\hline $\mathrm{F}=$ & \multicolumn{2}{|c|}{15,6920} \\
\hline $\mathrm{t}=$ & \multicolumn{2}{|c|}{$<042$} \\
\hline$p=$ & \multicolumn{2}{|c|}{$<0,0001$} \\
\hline
\end{tabular}

Tab. 20. Willendorf II, AH 6: Vergleich der SchlagflächenrestBreiten (in mm) zwischen allen in der Länge vollständig und proximal erhaltenen Lamellen bzw. Klingen sowie Absplissen bzw. Abschlägen (t-Test).
Tab. 21. Willendorf II, AH 6: Vergleich der SchlagflächenrestDicken (in mm) zwischen allen in der Länge vollständig und proximal erhaltenen Lamellen bzw. Klingen sowie Absplissen bzw. Abschlägen (t-Test). 


\begin{tabular}{|l|c|c|c|c|c|}
\hline Artefaktklasse AH 6 & $\begin{array}{c}\text { Komplett mit natürlicher } \\
\text { Oberfläche bedeckt }\end{array}$ & Dièdre & Linear & Glatt & Gesamt \\
\hline Klinge & - & - & - & 2 & 2 \\
\hline Lamelle & - & - & 12 & 8 & 20 \\
\hline Abschlag & 4 & 2 & 3 & 14 & $\mathbf{2 3}$ \\
\hline Abspliss & - & - & 1 & 2 & 3 \\
\hline Gesamt & 4 & 2 & 16 & $\mathbf{2 6}$ & $\mathbf{4 8}$ \\
\hline
\end{tabular}

Tab. 22. Willendorf II, AH 6: Art des Schlagflächenrestes bei allen in der Länge vollständig und proximal erhaltenen Grundformen.

\begin{tabular}{|l|c|c|c|c|c|c|c|c|}
\hline Artefakt AH 6 & Unbestimmbar & Unregelmäßig & Linear & Oval & Dreieckig & $\begin{array}{c}\text { Ventral } \\
\text { ausgesplittert }\end{array}$ & Geflügelt & Gesamt \\
\hline Klinge & - & 1 & - & 1 & - & - & - & 2 \\
\hline Lamelle & - & - & 12 & 4 & 2 & 1 & 1 & 20 \\
\hline Abschlag & 1 & 5 & 3 & 6 & - & 2 & 6 & 23 \\
\hline Abspliss & - & - & 1 & 1 & - & 1 & - & 3 \\
\hline Gesamt & 1 & $\mathbf{6}$ & 16 & 12 & $\mathbf{2}$ & 4 & 7 & 48 \\
\hline
\end{tabular}

Tab. 23. Willendorf II, AH 6: Form des Schlagflächenrestes bei allen in der Länge vollständig und proximal erhaltenen Grundformen.

\subsubsection{Schlagtechnische Charakteristika}

48 der Grundformen sind entweder vollständig oder proximal erhalten und konnten daher für die Bestimmung der Schlagmerkmale herangezogen werden. Das am häufigsten auftretende Schlagmerkmal stellt der Bulbus dar. Bei 97,9\% der Grundformen ist ein Bulbus vorhanden. Als zweithäufigstes Schlagmerkmal wurde die Lippe festgestellt. Insgesamt weisen 87,5\% der Grundformen eine Lippe auf. Sowohl die Abschläge und Absplisse als auch die Klingen und Lamellen besitzen hohe Anteile an Stücken mit Lippe. Schlagnarben treten bei 29,2 \% der Grundformen auf. Bei den Abschlägen gibt es tendenziell mehr Stücke mit Schlagnarbe als bei den Lamellen. Bei keiner einzigen Grundform wurde ein Kegel nachgewiesen. Neben den eben aufgezählten Schlagmerkmalen wurde bei den Grundformen auch ermittelt, ob eine dorsale Reduktion vorliegt oder nicht. Es zeigt sich, dass bei allen Klingen und Lamellen und bei einem Großteil der Abschläge und Absplisse diese Präparation an der ehemaligen Kante der Schlagfläche vorgenommen wurde. Außerdem wurden die Abbauwinkel gemessen. Bei den Abschlägen und Absplissen zeichnet sich eine größere Streuung in Bezug auf den Abbauwinkel ab, doch der Durchschnitt liegt wie bei Klingen und Lamellen bei $81^{\circ}$ und der Unterschied ist statistisch nicht signifikant (Tab. 24).

\begin{tabular}{|l|c|c|}
\hline AH 6 & Lamellen/Klingen & Absplisse/Abschläge \\
\hline Anzahl & 22 & 26 \\
\hline Mittelwert & 81,14 & 81,46 \\
\hline Stand. Abw. & 0,50 & 0,83 \\
\hline Varianz: & 1,27 & 4,18 \\
\hline $\mathrm{F}=$ & \multicolumn{2}{|c|}{3,2999} \\
\hline $\mathrm{t}=$ & \multicolumn{2}{|c|}{$-0,6650$} \\
\hline$p=$ & \multicolumn{3}{|c|}{0,5010} \\
\hline
\end{tabular}

Tab. 24. Willendorf II, AH 6: Vergleich des Abschlagwinkels (in Grad) aller in der Länge vollständig und proximal erhaltenen Lamellen bzw. Klingen sowie Absplisse bzw. Abschläge (t-Test).

Resümierend konnte für die Klingen und Lamellen zwar aufgrund der geringen Stückzahl nicht konkret entschieden werden, inwieweit der direkte, weiche, mineralische oder der direkte, organische Schlag zum Einsatz kam. Es ist jedoch möglich, den harten, direkten Schlag auszuschließen, denn (1) sind die Schlagflächenreste sehr dünn und schmal, (2) haben mit 54,5\% die meisten Schlagflächenreste eine lineare Form, (3) existiert mit 90,9 \% ein sehr hoher Anteil 
an Klingen und Lamellen mit Lippe, (4) treten Schlagnarben bei weniger als $30 \%$ auf, (5) wurde bei allen Klingen und Lamellen eine dorsale Reduktion festgestellt (somit wurden alle Schlagflächen der Kerne, von denen sie abgebaut wurden, vorher sorgfältig an der Kante präpariert), und (6) umfassen die gemessenen Abbauwinkel einen Bereich von 79 bis $83^{\circ}$, was nicht typisch für den direkten, harten Schlag ist. ${ }^{136}$

Nicht ganz so eindeutig kann eine Schlagtechnik bei den Abschlägen und Absplissen definitiv ausgeschlossen werden. In Bezug auf die Schlagflächenrest-Dicke zeigte sich, dass knapp 75 \% der Schlagflächenreste der Abschläge und Absplisse breiter als $1 \mathrm{~mm}$ sind und der Höchstwert bei $5 \mathrm{~mm}$ liegt. Außerdem gibt es unter den Abschlägen und Absplissen lediglich 15,4\% mit linearen, dagegen aber 61,5\% mit glatten Schlagflächenresten. Zwar liegen keine facettierten Schlagflächenreste vor, aber zwei Abschläge besitzen einen Schlagflächenrest des Typs dièdre. Des Weiteren weisen 84,6 \% der Abschläge und Absplisse Lippen auf. Schlagnarben treten bei weniger als $35 \%$ der Abschläge und bei keinem Abspliss auf. 73,1 \% der Abschläge und Absplisse weisen eine dorsale Reduktion auf. Zuletzt reichen die Abbauwinkel von 79 bis $87^{\circ}$, allerdings fallen $87 \%$ in denselben Bereich der Klingen und Lamellen. Vorzugsweise dürften auch die Abschläge und Absplisse mit direktem, weichem, mineralischem oder organischem Schlag hergestellt worden sein, aber aufgrund der teilweise mehrere Millimeter dicken Schlagflächenreste und der großen Abbauwinkel ist es naheliegend, dass einige Abschläge, die der Präparation des Kerns im Anfangsstadium des Abbaus dienten, durch den direkten, harten Schlag gewonnen wurden.

\subsubsection{Distalende}

Insgesamt gibt es 54 Grundformen, die entweder vollständig oder distal erhalten sind und Informationen zur Form des Distalendes geben (Tab. 25). Bei den Lamellen sind in der Seitenansicht 71,4 \% spitz zulaufend. Am häufigsten tritt die Kombination von sowohl in der Seiten- als auch in der Vorderansicht spitz zulaufenden Lamellen auf. Gerade oder spitz zulaufende Distalenden in der Vorderansicht, kombiniert mit spitz zulaufend oder gerundet in der Seitenansicht, sind typisch für Federbrüche. ${ }^{137}$ Somit kann von einer guten Präparation bzw. Instandhaltung der Kerne in Bezug auf die Abbaufläche und den Winkel zwischen Schlag- und Abbaufläche ausgegangen werden. Immerhin 23,8 \% der Lamellen besitzen einen Kernfuß. Diese Lamellen wurden über die ganze Abbaufläche hinweg abgebaut und entfernten einen

136 Pelegrin 2000.

137 Отт $1996,80$.
Teil vom gegenüberliegenden Ende des Kerns. Aufgrund des Auftretens von Lamellen mit diesem Charakteristikum dürfte ein Hinweis zum bereits fortgeschrittenen Verlauf des Abbaus und damit zur Abbaustrategie vorliegen (mehr dazu in Abschnitt 8.1).

Die einzige distal erhaltene Klinge weist in der Seitenansicht ein spitz zulaufendes Ende auf. In der Seitenansicht dominieren auch bei den Abschlägen die spitz zulaufenden. Allerdings gibt es keinen Abschlag, der in der Vorderansicht spitz zulaufend ist. Außerdem machen die Stücke mit Angelbruch bzw. Stufenbruch mehr als ein Drittel der Abschläge aus. Zwei Abschläge weisen distal einen Kernfuß auf. Da zumindest ein Teil der Abschläge der Präparation diente, dürfte bei diesen Stücken eher nicht auf die Einhaltung des optimalen Schlagwinkels oder eine ausreichend konvexe Abbaufläche geachtet worden sein, wodurch sich derartige Schlagunfälle ergeben. Auch ein zu großer Krafteinsatz könnte zu diesen Enden geführt haben.

Von den fünf in der Länge vollständig oder distal erhaltenen Absplissen sind $80 \%$ in der Seitenansicht spitz zulaufend, in der Vorderansicht jedoch kein einziger. Ein Abspliss endet in einem Angelbruch.

\subsubsection{Orientierung der dorsalen Negative}

Die Mehrheit der dorsalen Negative bei den Grundformen ist gleichgerichtet (Tab. 26). Dies spricht insgesamt dafür, dass der Abbau von einer bevorzugten Schlagfläche unidirektional vonstattenging. Einige der Grundformen wurden mit gleichgerichteten und quer zum Stück verlaufenden Negativen beobachtet. Dies dürfte einerseits mit der Kernkantenpräparation und andererseits mit der Präparation der Lateralflächen der Kerne zusammenhängen. Eine bidirektionale Orientierung der Negative kommt bei Lamellen und Abschlägen geringfügig vor.

\subsubsection{Querschnitte}

Bei den Lamellen und Abschlägen dominieren Stücke mit einem dreieckigen Querschnitt, bei den Klingen solche mit trapezförmigem Querschnitt (Tab. 27). Insgesamt machen dreieckige und trapezförmige Querschnitte den Großteil des Inventars aus. Lediglich bei den Abschlägen und Absplissen treten Stücke mit unregelmäßigem Querschnitt auf. Somit zeigt sich, dass die Kerne sorgfältig durch ein regelmäßiges Gratmuster organisiert worden sein dürften.

\subsubsection{Zusammenpassungen}

Nachdem innerhalb der bereits vorsortierten Rohmaterialgruppen systematische Untersuchungen durchgeführt worden waren, um unter den Artefakten des Inventars $\mathrm{Zu}$ sammenpassungen zu finden, konnten 19 Grundformen 


\begin{tabular}{|l|c|c|c|c|c|c|}
\hline Seitenansicht/Vorderansicht & Konkav & Konvex & Unregelmäßig & Spitz zulaufend & Gerade & Gesamt \\
\hline Angelbruch & - & 5 & 1 & - & 5 & 11 \\
\hline Kernfuß & - & 3 & 2 & - & 2 & 7 \\
\hline Spitz zulaufend & 3 & 8 & 9 & 9 & 4 & 33 \\
\hline Gerundet & - & - & 1 & - & 1 & 2 \\
\hline Stufenbruch & - & - & - & - & 1 & 1 \\
\hline Gesamt & 3 & 16 & 13 & 9 & 13 & 54 \\
\hline
\end{tabular}

Tab. 25. Willendorf II, AH 6: Form des Distalendes bei allen in der Länge vollständig oder distal erhaltenen Grundformen.

\begin{tabular}{|l|c|c|c|c|c|c|c|c|c|c|c|c|c|c|c|c|}
\hline $\begin{array}{l}\text { Artefaktklasse } \\
\text { AH 6 }\end{array}$ & Gleichgerichtet & \multicolumn{2}{|c|}{$\begin{array}{c}\text { Quer } \\
\text { einseitig }\end{array}$} & \multicolumn{2}{|c|}{ Bidirektional } & \multicolumn{2}{|c|}{ Kluft } & \multicolumn{2}{|c|}{ Gegenläufig } & \multicolumn{2}{|c|}{$\begin{array}{c}\text { Gleichgerichtet } \\
\text { und quer }\end{array}$} & \multicolumn{2}{|c|}{$\begin{array}{l}\text { Unbestimmte } \\
\text { Schlagrichtung }\end{array}$} & \multicolumn{2}{c|}{ Gesamt } \\
\hline & $\mathrm{n}$ & $\%$ & $\mathrm{n}$ & $\%$ & $\mathrm{n}$ & $\%$ & $\mathrm{n}$ & $\%$ & $\mathrm{n}$ & $\%$ & $\mathrm{n}$ & $\%$ & $\mathrm{n}$ & $\%$ & $\mathrm{n}$ & $\%$ \\
\hline Klinge & 5 & $71,4 \%$ & - & - & - & - & - & - & - & - & 2 & $28,6 \%$ & - & - & 7 & $100 \%$ \\
\hline Lamelle & 28 & $70 \%$ & - & - & 1 & $2,5 \%$ & 1 & $2,5 \%$ & - & - & 9 & $22,5 \%$ & 1 & $2,5 \%$ & 40 & $100 \%$ \\
\hline Abschlag & 28 & $65,1 \%$ & 1 & $2,3 \%$ & 2 & $4,7 \%$ & 1 & $2,3 \%$ & 1 & $2,3 \%$ & 10 & $23,3 \%$ & - & - & 43 & $100 \%$ \\
\hline Abspliss & 3 & $30 \%$ & - & - & 2 & $20 \%$ & - & - & - & - & 2 & $20 \%$ & 3 & $30 \%$ & 10 & $100 \%$ \\
\hline Gesamt & $\mathbf{6 4}$ & $\mathbf{6 4} \%$ & 1 & $\mathbf{1} \%$ & $\mathbf{5}$ & $\mathbf{5} \%$ & $\mathbf{2}$ & $\mathbf{2} \%$ & 1 & $1 \%$ & 23 & $23 \%$ & 4 & $4 \%$ & 100 & $100 \%$ \\
\hline
\end{tabular}

Tab. 26. Willendorf II, AH 6: Orientierung der Negative bei allen Grundformen.

\begin{tabular}{|l|c|c|c|c|c|c|}
\hline Artefaktklasse AH 6 & Unbestimmbar & Unregelmäßig & Linsenförmig & Trapezförmig & Dreieckig & Gesamt \\
\hline Klinge & 1 & - & - & 3 & 2 & $\mathbf{6}$ \\
\hline Lamelle & - & - & 1 & 10 & 17 & $\mathbf{2 8}$ \\
\hline Abschlag & - & 4 & 5 & 4 & 11 & $\mathbf{2 4}$ \\
\hline Abspliss & - & 2 & 1 & 1 & - & 4 \\
\hline Gesamt & 1 & $\mathbf{6}$ & $\mathbf{7}$ & $\mathbf{1 8}$ & $\mathbf{3 0}$ & $\mathbf{6 2}$ \\
\hline
\end{tabular}

Tab. 27. Willendorf II, AH 6: Querschnitte bei allen in der Breite vollständigen Grundformen.

aus dem Inventar von AH 6 zusammengepasst werden. Damit liegt der Zusammenpassungsanteil des Inventars bei 18,6 \%. Vergleichsweise zeigt das magdalénienzeitliche Inventar des Grabungsbereichs U5 aus der Fundstelle Étiolles, Frankreich, ebenfalls einen durchschnittlichen Zusammenpassungsanteil von 18,6\%. ${ }^{138}$ In AH 6 fanden sich acht Zusammenpassungskomplexe (Tab. 28). Sechs Komplexe setzen sich aus zwei Stücken zusammen und jeweils ein Komplex umfasst drei bzw. vier Stücke.

138 Pigeot 1987. - Bar-Yosef, Van Peer 2009, 106.

\subsubsection{Zusammenpassungstypen}

Es kommen ausschließlich Aufeinander- und Aneinanderpassungen vor, somit konnte die Grundformenherstellung vor Ort ganz klar belegt werden. Das Fehlen von Anpassungen von Werkzeugabfällen auf modifizierte Grundformen heißt natürlich nicht, dass keine Modifikation der Grundformen an der Fundstelle stattfand. Es konnte lediglich kein Nachweis für die Werkzeugproduktion erbracht werden. Mögliche Gründe dafür könnten sein, dass die in die kleinste Größenklasse fallenden Werkzeugabfälle aufgrund der 


\begin{tabular}{|c|c|c|c|}
\hline 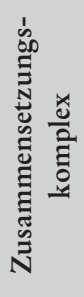 & 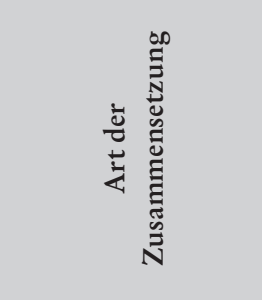 & 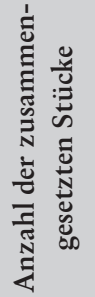 & 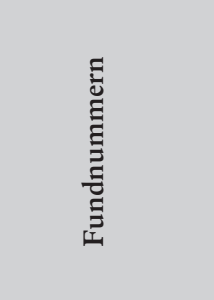 \\
\hline 001 & Aneinanderpassung & 2 & $\begin{array}{l}\text { WII-1993-157-8 } \\
\text { WII-1993-158-15 }\end{array}$ \\
\hline 003 & Aneinanderpassung & 2 & $\begin{array}{l}\text { WII-1993-171-4 } \\
\text { WII-1993-171-5 }\end{array}$ \\
\hline 004 & Aufeinanderpassung & 4 & $\begin{array}{c}\text { WII-1993-158-6 } \\
\text { WII-1993-158-12 } \\
\text { WII-1993-159-2 } \\
\text { WII-1993-171-34 }\end{array}$ \\
\hline 005 & Aufeinanderpassung & 2 & $\begin{array}{l}\text { WII-1993-158-7 } \\
\text { WII-1993-158-9 }\end{array}$ \\
\hline 006 & Aufeinanderpassung & 3 & $\begin{array}{l}\text { WII-1993-171-1 } \\
\text { WII-1993-171-2 } \\
\text { WII-1993-171-3 }\end{array}$ \\
\hline 007 & Aufeinanderpassung & 2 & $\begin{array}{l}\text { WII-1993-158-10 } \\
\text { WII-1993-170-3 }\end{array}$ \\
\hline 008 & Aufeinanderpassung & 2 & $\begin{array}{l}\text { WII-1993-171-8 } \\
\text { WII-1993-171-31 }\end{array}$ \\
\hline 027 & Aneinanderpassung & 2 & $\begin{array}{l}\text { WII-1993-163-1 } \\
\text { WII-1993-163-2 }\end{array}$ \\
\hline
\end{tabular}

Tab. 28. Willendorf II, AH 6: Zusammensetzungskomplexe der Grundformen.

Tatsache, dass nicht geschlämmt wurde, nicht aufgefunden wurden, oder dass der Grabungsbereich eine Zone darstellt, in welcher diese Aktivität nicht durchgeführt wurde.

Zusammenpassungen wurden bei allen drei Hauptrohmaterialien gefunden. Sowohl bei dem Zusammenpassungskomplex aus baltischem Feuerstein als auch bei jenem aus Radiolarit handelt sich um Aneinanderpassungen. Der überwiegende Anteil (78,9\%) an zusammengepassten Grundformen ist aus alpinem Hornstein. Abschläge und Lamellen stellen die Grundformen dar, die zusammengepasst werden konnten. Der Anteil von 52,6 \% der zusammengepassten Abschläge ist geringfügig höher als der Anteil der zusammengepassten Lamellen.

Nun folgt eine kurze Beschreibung der für den Abbauprozess aussagekräftigsten Zusammenpassungen.

Zusammenpassungskomplex 004: Drei Lamellen und ein Abschlag aus alpinem Hornstein konnten aufeinandergepasst werden, wobei der Abschlag in dieser Abbausequenz zuletzt gelöst wurde. Von dem Abschlag und einer der Lamellen liegt das Distalende vor. In beiden Fällen handelt es sich um Angelbrüche. Von den weiteren Lamellen ist die eine medial und die andere proximal erhalten. Das Proximalende ist linear und wurde reduziert. Alle Elemente dieser Aufeinanderpassung sind dextrolateral komplett mit natürlichen Oberflächen bedeckt. Außerdem weisen alle Stücke dorsal gleichgerichtete Negative auf. Einerseits konnte durch diesen Zusammenpassungskomplex der unidirektionale Abbau bestätigt werden und andererseits belegen die Abbauprodukte mit ihren dextrolateralen Bereichen von natürlichen Oberflächen, dass die Abbaufläche zur rechten Flanke hin erweitert wurde. Damit könnte ein Hinweis für folgendes Abbauszenario vorliegen: Nachdem der Abbau initialisiert wurde, erfolgte die Erweiterung auf die Flanken, wobei der Grat, der sich zwischen den in die Flanken übergehenden und den frontalen Negativen ergibt, als Leitgrat für die Gewinnung der Zielprodukte gedient haben dürfte. ${ }^{139}$

Zusammenpassungskomplex 005: Hierbei gelang eine Aufeinanderpassung zwischen zwei Abschlägen aus alpinem Hornstein. Beide weisen dorsal keine natürlichen Oberflächen auf. Das aufgesetzte Stück ist distal erhalten, während das andere Stück vollständig ist. Beide Grundformen enden in einem Kernfuß. Der Schlagflächenrest des vollständig erhaltenen Abschlags ist glatt und oval. Außerdem konnte eine dorsale Reduktion festgestellt werden. Die dorsalen Negative verlaufen gleichgerichtet und quer. Es dürfte sich um Abschläge handeln, die der lateralen Präparation des Kerns in einer schon fortgeschrittenen Abbauphase dienten.

Zusammenpassungskomplex 006: Zwei Lamellen aus alpinem Hornstein wurden auf einen Abschlag aufgesetzt. Alle Stücke sind vollständig erhalten und enden spitz zulaufend. Bei allen wurde eine dorsale Reduktion festgestellt, doch die Form des Schlagflächenrestes ist unterschiedlich. Eine der Lamellen weist einen linearen Schlagflächenrest auf, während die andere einen glatten, ovalen und der Abschlag einen glatten, geflügelten Schlagflächenrest besitzt. Keine der Grundformen zeigt Reste natürlicher Oberflächen auf der Dorsalfläche. Bei beiden Lamellen verlaufen die dorsalen Negative gleichgerichtet und quer, womit wiederum ein Beleg für die laterale Kernpräparation vorliegen dürfte. Die dorsalen Negative des Abschlags sind ausschließlich gleichgerichtet.

Zusammenpassungskomplex 007 (Abb. 8): Eine Lamelle aus alpinem Hornstein wurde auf eine andere Lamelle aufgesetzt. Beide sind vollständig erhalten und enden gerade bzw. konvex. Beide weisen eine dorsale Reduktion

139 Siehe Moreau 2009, 72. 


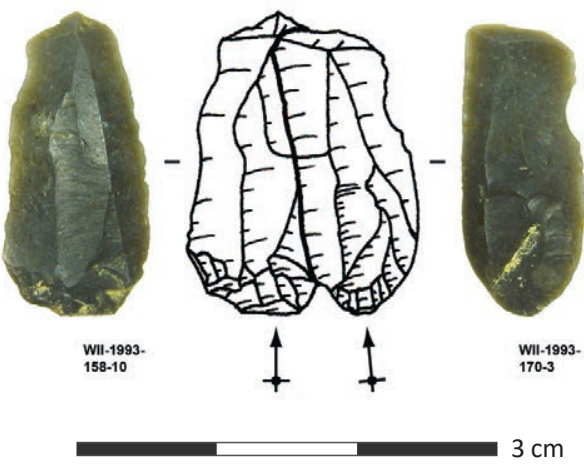

Abb. 8. Willendorf II, AH 6: Zusammenpassungskomplex 007 (Zeichnung: F. Brodbeck).

und lineare Schlagflächenreste auf. Es sind keine Reste natürlicher Oberflächen auf der Dorsalfläche vorhanden. Bei beiden Lamellen verlaufen die dorsalen Negative gleichgerichtet und quer, womit ein weiteres Mal die laterale Kernpräparation belegt ist.

Die Zusammenpassungen konnten Informationen über die Abbaustrategie liefern. Von den acht Zusammenpassungskomplexen wurden fünf jeweils innerhalb eines Sammelfundes gefunden, daher müssen sich die Stücke in unmittelbarer Nähe zueinander befunden haben. Bei einer Aneinanderpassung stammen beide Fragmente aus derselben Höhe, allerdings erlauben die weiteren Angaben keine Aussagen zur horizontalen Verteilung. Des Weiteren setzt sich eine Aufeinanderpassung aus einem Artefakt mit Schichtangabe AH 6 und einem Artefakt, das von der Höhenangabe her eindeutig in den Bereich von AH 6 fällt, zusammen. Zuletzt umfasst der aus vier Stücken bestehende Komplex zwei Stücke aus demselben Sammelfund, ein Stück, das im Höhenbereich der Stücke aus dem Sammelfund liegt, und ein Stück mit der Schichtangabe AH 6.

\subsubsection{Werkzeuge}

Von den 102 vorliegenden Grundformen wurden acht Stücke und damit 7,8 \% insgesamt modifiziert. Vier Werkzeugtypen (Stichel, Kantenretusche, rückengestumpfte Formen und Kratzer) sind gleich stark vertreten (Tab. 29). Wie bereits bei den Rohmaterialien erwähnt, wurden die Rückenelemente aus den qualitativ hochwertigen Rohmaterialien Hornstein und baltischer Feuerstein, die allerdings auch am häufigsten vorkommen, hergestellt. Auch für die Stichel und die Kantenretuschen wurden ausschließlich diese Rohmaterialien herangezogen. Von den beiden Kratzern wurde der eine aus Radiolarit und der andere aus Quarzit gefertigt. Insgesamt wurden Klingen und Lamellen häufiger in Werkzeuge umgearbeitet als Abschläge. Trotz der geringen Stückzahl zeigt sich, dass ausschließlich Lamellen

\begin{tabular}{|l|c|}
\hline Werkzeugtyp AH 6 & Gesamt \\
\hline Stichel & 2 \\
\hline Kantenretusche & 2 \\
\hline Rückengestumpftes Element & 2 \\
\hline Kratzer & 2 \\
\hline Gesamt & $\mathbf{8}$ \\
\hline
\end{tabular}

Tab. 29. Willendorf II, AH 6: Typologische Zusammensetzung der Werkzeuge.

zu Rückenelementen verarbeitet wurden. Kantenretuschen finden sich sowohl an Lamellen als auch Klingen. Die Stichel wurden an Klingen und die Kratzer an Abschlägen hergestellt. Daher kann davon ausgegangen werden, dass für Projektile längliche Grundformen gezielt produziert wurden, während hingegen für andere Werkzeuge wie Kratzer die Morphologie des Ausgangsstückes weniger relevant gewesen sein dürfte. Folgende Werkzeugtypen sind im Inventar von AH 6 vorhanden:

Stichel: Einer der beiden Stichel ist ein einfacher Stichel. Die Stichelbahn dieses Stichels aus alpinem Hornstein ist nicht vollständig, insofern kann nicht ausgesagt werden, ob es sich um einen Stichel an Bruch, Endretusche oder natürlicher Fläche handelt. Der Stichel aus baltischem Feuerstein steht im Zusammenhang mit einem Schlagunfall. Zwei Stichelbahnen wurden an einer konkaven Endretusche angebracht, wobei, nachdem die erste Stichelbahn mit einer Breite von 3,3 mm dextrolateral angelegt worden war, die Endretusche für den zweiten Stichelschlag nachpräpariert wurde und dadurch die Länge der ersten Bahn nicht mehr vollständig ist. Mit der zweiten Stichelbahn, die 6,3 mm breit und 45,8 mm lang ist, wurde der Schlagunfall, ein sogenannter outrepassé, verursacht, damit wurde die distal für die Sticheltechnik angebrachte Schlagfläche entfernt und es blieb lediglich dextrolateral der Rest einer ehemaligen Stichelbahn von distal erhalten.

Kantenretuschen: Beide Kantenretuschen sind in der Länge gebrochen und besitzen außerdem lediglich eine retuschierte Kante. Bei der Kantenretusche an der Lamelle wurde eine gerade, fein- und scharfkantige Retusche mit einer Höhe von 0,9 mm angelegt. Die Klinge wurde mit einer starken, 0,5 mm hohen Kantenretusche, die schräg gerade verläuft, versehen.

Rückenelemente: Bei dem Rückenelement aus alpinem Hornstein handelt es sich um eine Lamelle mit einfachem Rücken (Abb. 6/WII-1993-171-24). Eine Lamelle wird dann diesem Werkzeugtyp zugesprochen, wenn sie über einen geraden, durch abrupte Retusche angefertigten Rücken 
verfügt, wobei aber im Gegensatz zu einem klassischen Rückenmesser der Schlagflächenrest bzw. das Distalende der ursprünglichen Grundform noch erhalten ist. ${ }^{140}$ Dieses Werkzeug ist vollständig erhalten. Die Länge der Modifikation beträgt 13,4 mm und die Höhe 0,3 mm. Bei der rückengestumpften Form aus baltischem Feuerstein handelt es sich um eine Lamelle mit beidseitigem Rücken (Abb. 6/ WII-1993-171-22). Wie bei der Lamelle mit einfachem Rücken besitzt auch diese Lamelle einen geraden, durch abrupte Retusche angefertigten Rücken dextrolateral und ist proximal erhalten, allerdings existiert zusätzlich sinistrolateral ein schräg gerader, durch abrupte Retusche hergestellter Rücken. Beide Rückenstumpfungen sind in der Länge nicht vollständig erhalten. Dextrolateral ist die Modifikation 1,4 mm und sinistrolateral 0,8 $\mathrm{mm}$ hoch.

Kratzer: Beide Kratzer wurden als einfache Kratzer angesprochen und in beiden Fällen liegt eine konvexe Retusche, deren Länge nicht vollständig erhalten ist, vor. Bei dem einfachen Kratzer aus Radiolarit wurde die Kratzerkappe mit einer Höhe von 8,3 $\mathrm{mm}$ am Proximalende angelegt. Die 2,6 mm hohe Kratzerkappe des einfachen Kratzers aus Quarzit befindet sich distal.

\subsubsection{Erhaltungszustand}

Insgesamt sind die meisten Grundformen gebrochen und nur $31 \%$ vollständig erhalten (Tab. 30). Unter den ohnedies nicht so zahlreich vorhandenen Klingen findet sich kein einziges in der Länge vollständig erhaltenes Stück, mehr als $50 \%$ der Klingen sind medial erhalten. Mit jeweils mehr als einem Drittel machen bei Lamellen und Abschlägen den größten Anteil die in der Länge vollständig erhaltenen aus. Die medial erhaltenen Lamellen umfassen immerhin auch fast ein Drittel. Distal- und Proximalfragmente bei den Lamellen liegen bei ca. $15 \%$. Bei den Abschlägen sind am zweithäufigsten die Distalfragmente vertreten. Bei den Absplissen gibt es nur ein vollständig erhaltenes Stück und es dominieren ebenfalls die Distalfragmente.

$75 \%$ der Grundformen sind sowohl in der Breite als auch in der Dicke vollständig erhalten (Tab. 31). 14,3 \% der Klingen und $10 \%$ der Lamellen sind lateral gebrochen. Bei den Abschlägen dominieren auch die in der Breite und Dicke vollständig erhaltenen mit 67,4\%. Aber der Anteil an den lateral gebrochenen Abschlägen fällt mit 30,2 \% höher aus als bei Klingen und Lamellen. Bei den Absplissen sind die meisten in der Breite unvollständig.

Insgesamt weisen $57 \%$ der Grundformen eine Kantenbeschädigung auf. Ein Anteil von 8,8 \% der Grundformen hat thermische Veränderungen. Die im Inventar

140 Moreau 2009, 40. nachgewiesenen thermischen Einwirkungen deuten zum Großteil auf Hitzebeschädigungen und damit eher auf einen zufälligen Kontakt mit Feuer hin.

\subsection{Ergebnisse zur Steintechnologie in AH 8}

\subsubsection{Grundformen}

\subsubsection{Grundformenanteile}

Bei den Grundformen machen die Abschläge mit 42,3\% den größten Anteil aus (Tab. 12). Die Klingen sind mit 15,4 \% klar stärker vertreten als die Lamellen mit 3,6 \%. Es liegen keine Absplisse vor. Da nicht geschlämmt wurde, könnten die kleinen Artefaktklassen (Lamellen, Absplisse) unterrepräsentiert sein. In AH 6 jedoch wurden sehr wohl Absplisse während der Grabungstätigkeit gefunden, womit deren Fehlen in AH 8 zumindest nicht ausschließlich als ein Grabungsartefakt anzusehen ist. Die Kernabbaustrategie dürfte durch die starke Präsenz an Klingen anstelle von Lamellen eher auf größere Produkte abgezielt haben.

Kernkantenabschläge, Kernkantenklingen und Kernkantenlamellen treten zwar nur geringfügig auf, aber dennoch stellen sie den Beleg für diese bestimmte Form der Kernpräparation dar. Kernscheiben zur Korrektur des Abbauwinkels während des Kernabbaus, die keine scharfen Kanten, sondern einen durch senkrecht zur Ventralfläche verlaufende Negative gebildeten Rand besitzen, ${ }^{141}$ sind ebenfalls vorhanden. Kerne und Kerntrümmer umfassen zusammen 5,9 \% des Gesamtmaterials. Der Anteil an Trümmern liegt bei 3,6 \%. Neun Abschläge konnten aufgrund der charakteristischen, ventral sowie dorsal ausgesplitterten Schlagflächenreste als Abfälle von Ausgesplitterten Stücken identifiziert werden. Oftmals treten auch distal Aussplitterungen auf. ${ }^{142}$ Die Herstellung dieser Werkzeugform ereignete sich somit vor Ort (genauere Angaben folgen in Abschnitt 7.3.3.1).

\subsubsection{Maße}

Die Längenverteilung zeigt, dass keine einzige in der Länge vollständig erhaltene Grundform eine Länge unter $10 \mathrm{~mm}$ aufweist (Tab. 32). Vier Abschläge kommen auf eine Länge von über $70 \mathrm{~mm}$ und zwei davon sogar über $80 \mathrm{~mm}$. Wenn die Längenverteilung je nach Grundform - wobei keine einzige Lamelle in der Länge vollständig erhalten ist - betrachtet wird, so zeigt sich, dass die Abschläge von 10,4 bis $86,5 \mathrm{~mm}$ streuen, während bei den Klingen eine Verteilung von 38,2 bis $60,8 \mathrm{~mm}$ festgestellt wurde (Tab. 33). Die

141 Hahn 1993, 104.

142 Villa, Delagnes, Wadley 2005, 413. 


\begin{tabular}{|l|c|c|c|c|c|c|c|c|c|c|}
\hline \multirow{2}{*}{ Artefaktklasse AH 6 } & \multicolumn{2}{|c|}{ Vollständig } & \multicolumn{2}{|c|}{ Proximal } & \multicolumn{2}{c|}{ Medial } & \multicolumn{2}{c|}{ Distal } & \multicolumn{3}{c|}{ Gesamt } \\
\hline & $\mathrm{n}$ & $\%$ & $\mathrm{n}$ & $\%$ & $\mathrm{n}$ & $\%$ & $\mathrm{n}$ & $\%$ & $\mathrm{n}$ & $\%$ \\
\hline Klinge & - & - & 2 & $28,6 \%$ & 4 & $57,1 \%$ & 1 & $14,3 \%$ & 7 & $100 \%$ \\
\hline Lamelle & 14 & $35 \%$ & 6 & $15 \%$ & 13 & $32,5 \%$ & 7 & $17,5 \%$ & 40 & $100 \%$ \\
\hline Abschlag & 16 & $37,2 \%$ & 7 & $16,3 \%$ & 9 & $20,9 \%$ & 11 & $25,6 \%$ & 43 & $100 \%$ \\
\hline Abspliss & 1 & $10 \%$ & 2 & $20 \%$ & 3 & $30 \%$ & 4 & $40 \%$ & 10 & $100 \%$ \\
\hline Gesamt & 31 & $31 \%$ & 17 & $17 \%$ & 29 & $29 \%$ & 23 & $23 \%$ & 100 & $100 \%$ \\
\hline
\end{tabular}

Tab. 30. Willendorf II, AH 6: Erhaltung der Länge (in mm) bei allen Grundformen.

\begin{tabular}{|l|c|c|c|c|c|c|c|c|c|c|}
\hline \multirow{2}{*}{ Artefaktklasse AH 6 } & \multicolumn{2}{|c|}{$\begin{array}{c}\text { Breite } \\
\text { vollständig }\end{array}$} & \multicolumn{2}{c|}{$\begin{array}{c}\text { Breite } \\
\text { unvollständig }\end{array}$} & \multicolumn{2}{c|}{$\begin{array}{c}\text { Dicke } \\
\text { unvollständig }\end{array}$} & \multicolumn{2}{c|}{$\begin{array}{c}\text { Breite und } \\
\text { Dicke unvollständig }\end{array}$} & \multicolumn{2}{c|}{ Gesamt } \\
\hline & $\mathrm{n}$ & $\%$ & $\mathrm{n}$ & $\%$ & $\mathrm{n}$ & $\%$ & $\mathrm{n}$ & $\%$ & $\mathrm{n}$ & $\%$ \\
\hline Klinge & 6 & $85,7 \%$ & 1 & $14,3 \%$ & - & - & - & - & 7 & $100 \%$ \\
\hline Lamelle & 36 & $90 \%$ & 4 & $10 \%$ & - & - & - & - & 40 & $100 \%$ \\
\hline Abschlag & 29 & $67,4 \%$ & 13 & $30,2 \%$ & - & - & 1 & $2,3 \%$ & 43 & $100 \%$ \\
\hline Abspliss & 4 & $40 \%$ & 5 & $50 \%$ & - & - & 1 & $10 \%$ & 10 & $100 \%$ \\
\hline Gesamt & 75 & $75 \%$ & 23 & $23 \%$ & - & - & 2 & $2 \%$ & 100 & $100 \%$ \\
\hline
\end{tabular}

Tab. 31. Willendorf II, AH 6: Erhaltung der Breite und Dicke bei allen Grundformen.

\begin{tabular}{|l|c|}
\hline AH 8 & Grundformen \\
\hline Anzahl & 54 \\
\hline Min & 10,4 \\
\hline Max & 86,45 \\
\hline Mittelwert & 37,33 \\
\hline Stand. Abw. & 19,56 \\
\hline Median & 32,37 \\
\hline
\end{tabular}

\begin{tabular}{|l|c|c|}
\hline AH 8 & Klingen & Abschläge \\
\hline Anzahl & 6 & 48 \\
\hline Min & 38,15 & 10,4 \\
\hline Max & 60,84 & 86,45 \\
\hline Mittelwert & 50,15 & 35,73 \\
\hline Stand. Abw. & 10,04 & 19,93 \\
\hline Median & 49,64 & 30,41 \\
\hline
\end{tabular}

Tab. 32. Willendorf II, AH 8: Deskriptive Statistik der Länge (in $\mathrm{mm}$ ) aller in der Länge vollständig erhaltenen Grundformen.

geringe Stückzahl der in der Länge vollständig erhaltenen Klingen aus $\mathrm{AH} 8$ ist jedoch nicht besonders aussagekräftig, um auf eine etwaige Standardisierung schließen zu können.

Die Breite der Grundformen liegt zwischen 5,4 und $81,1 \mathrm{~mm}$ (Tab. 34), jedoch mehr als $75 \%$ der Grundformen sind nicht breiter als $30 \mathrm{~mm}$ (Abb. 9). Bei der Breitenverteilung unterteilt nach Abschlägen und Klingen bzw. Lamellen zeigt sich, dass die Abschläge, wie auch schon bei der Längenverteilung, eine wesentlich größere Spanne als die Klingen und Lamellen aufweisen (Tab. 35). Ein Abschlag

Tab. 33. Willendorf II, AH 8: Deskriptive Statistik der Länge (in $\mathrm{mm}$ ) aller in der Länge vollständig erhaltenen Klingen und Abschläge.

\begin{tabular}{|l|c|}
\hline AH 8 & Grundformen \\
\hline Anzahl & 99 \\
\hline Min & 5,38 \\
\hline Max & 81,13 \\
\hline Mittelwert & 24,07 \\
\hline Stand. Abw. & 16,66 \\
\hline Median & 18,18 \\
\hline
\end{tabular}

Tab. 34. Willendorf II, AH 8: Deskriptive Statistik der Breite (in $\mathrm{mm}$ ) aller in der Breite vollständig erhaltenen Grundformen. 
überschreitet den Wert von $80 \mathrm{~mm}$. Die Breitenverteilung von 44 der insgesamt 45 in der Breite vollständig erhaltenen Klingen bzw. Lamellen liegt zwischen 5,4 und 25,1 mm; lediglich eine Klinge liegt weit außerhalb dieser Spanne bei $39,6 \mathrm{~mm}$. Zehn Stücke (22,2 \%) haben eine Breite von unter $10 \mathrm{~mm}$ und entsprechen demnach der Definition von Lamellen.

\begin{tabular}{|l|c|c|}
\hline AH 8 & Lamellen/Klingen & Abschläge \\
\hline Anzahl & 45 & 54 \\
\hline Min & 5,38 & 8,98 \\
\hline Max & 39,59 & 81,13 \\
\hline Mittelwert & 14,8 & 31,8 \\
\hline Stand. Abw. & 5,78 & 18,75 \\
\hline Median & 14,43 & 28,03 \\
\hline
\end{tabular}

Tab. 35. Willendorf II, AH 8: Deskriptive Statistik der Breite (in $\mathrm{mm}$ ) aller in der Breite vollständig erhaltenen Klingen und Lamellen sowie Abschläge.

Keine einzige Grundform hat eine Dicke von unter $1 \mathrm{~mm}$ (Tab. 36). 77,6\% der Grundformen jedoch überschreiten den Wert von $10 \mathrm{~mm}$ nicht und lediglich fünf Abschläge weisen eine Dicke von mehr als $20 \mathrm{~mm}$ auf. Anhand der Dickenverteilung aufgegliedert nach Abschlägen und Klingen bzw. Lamellen wird wiederum die wesentlich größere Streuung der Abschläge von 1,6 bis 28,8 mm gegenüber den Klingen und Lamellen von 1,9 bis $12,8 \mathrm{~mm}$ deutlich (Tab. 37). Immerhin sind 69,1 \% der Abschläge nicht dicker als 10 mm. $75 \%$ der Klingen und Lamellen streuen zwischen 1,9 und 6,1 mm, lediglich drei Klingen liegen mit ihrer Dicke bei über $10 \mathrm{~mm}$.

\begin{tabular}{|l|c|}
\hline AH 8 & Grundformen \\
\hline Anzahl & 147 \\
\hline Min & 1,62 \\
\hline Max & 28,79 \\
\hline Mittelwert & 7,38 \\
\hline Stand. Abw. & 4,99 \\
\hline Median & 5,76 \\
\hline
\end{tabular}

Tab. 36. Willendorf II, AH 8: Deskriptive Statistik der Dicke (in $\mathrm{mm}$ ) aller in der Dicke vollständig erhaltenen Grundformen.

\begin{tabular}{|l|c|c|}
\hline AH 8 & Lamellen/Klingen & Abschläge \\
\hline Anzahl & 50 & 97 \\
\hline Min & 1,85 & 1,62 \\
\hline Max & 12,83 & 28,79 \\
\hline Mittelwert & 5 & 8,61 \\
\hline Stand. Abw. & 2,46 & 5,5 \\
\hline Median & 4,21 & 7,5 \\
\hline
\end{tabular}

Tab. 37. Willendorf II, AH 8: Deskriptive Statistik der Dicke (in $\mathrm{mm}$ ) aller in der Dicke vollständig erhaltenen Klingen und Lamellen sowie Abschläge.

\subsubsection{Dorsale Bedeckung mit natürlichen Oberflächen}

Mehr als zwei Drittel der Klingen und Lamellen weisen auf der Dorsalfläche keine Reste von natürlichen Oberflächen auf (Tab. 38). Von den Abschlägen hingegen haben nur 37,8 \% dorsal keine Bedeckung mit natürlichen Oberflächen. Dies deutet darauf hin, dass in einer anfänglichen Phase des Kernabbaus Abschläge dorsal komplett bedeckt mit natürlichen Oberflächen abgetrennt wurden, um erstens den Kern zu entrinden und zweitens den Kern für die anschließende Produktion von Klingen zu präparieren. Anhand der Lage der natürlichen Oberflächen zeichnet sich kein konkretes Muster ab, da alle Lagen mehr oder weniger stark vertreten vorkommen.

2,3 \% der Klingen und 6,7 \% der Abschläge sind komplett mit natürlichen Oberflächen bedeckt und bilden damit die primären Grundformen. Somit kann davon ausgegangen werden, dass zumindest teilweise Rohknollen vor der Entrindung zur Fundstelle gebracht wurden und dann dort alle Stadien der Operationskette durchgeführt wurden.

\subsubsection{Schlagflächenreste}

Die Schlagflächenrest-Breite und Schlagflächenrest-Dicke zeigen, dass bei Klingen und Lamellen die Schlagflächenreste eindeutig kleiner und schmaler sind als bei den Abschlägen (Tab. 39, 40) und außerdem die Streuung wesentlich geringer ausfällt (Abb. 10).

In $\mathrm{AH} 8$ weisen 48,5\% aller Grundformen einen glatten und 33,3\% einen Schlagflächenrest bedeckt mit natürlichen Oberflächen auf (Tab. 41). Bei den Lamellen wurden ausschließlich Stücke mit glattem Schlagflächenrest nachgewiesen. 76,2 \% der Klingen verfügen ebenfalls über einen glatten Schlagflächenrest. Allerdings gibt es auch zwei Klingen mit Schlagflächenrest bedeckt mit natürlichen Oberflächen und eine Klinge mit facettiertem Schlagflächenrest. Bei den Abschlägen ist der Schlagflächenrest vorwiegend mit natürlichen Oberflächen bedeckt oder glatt. 


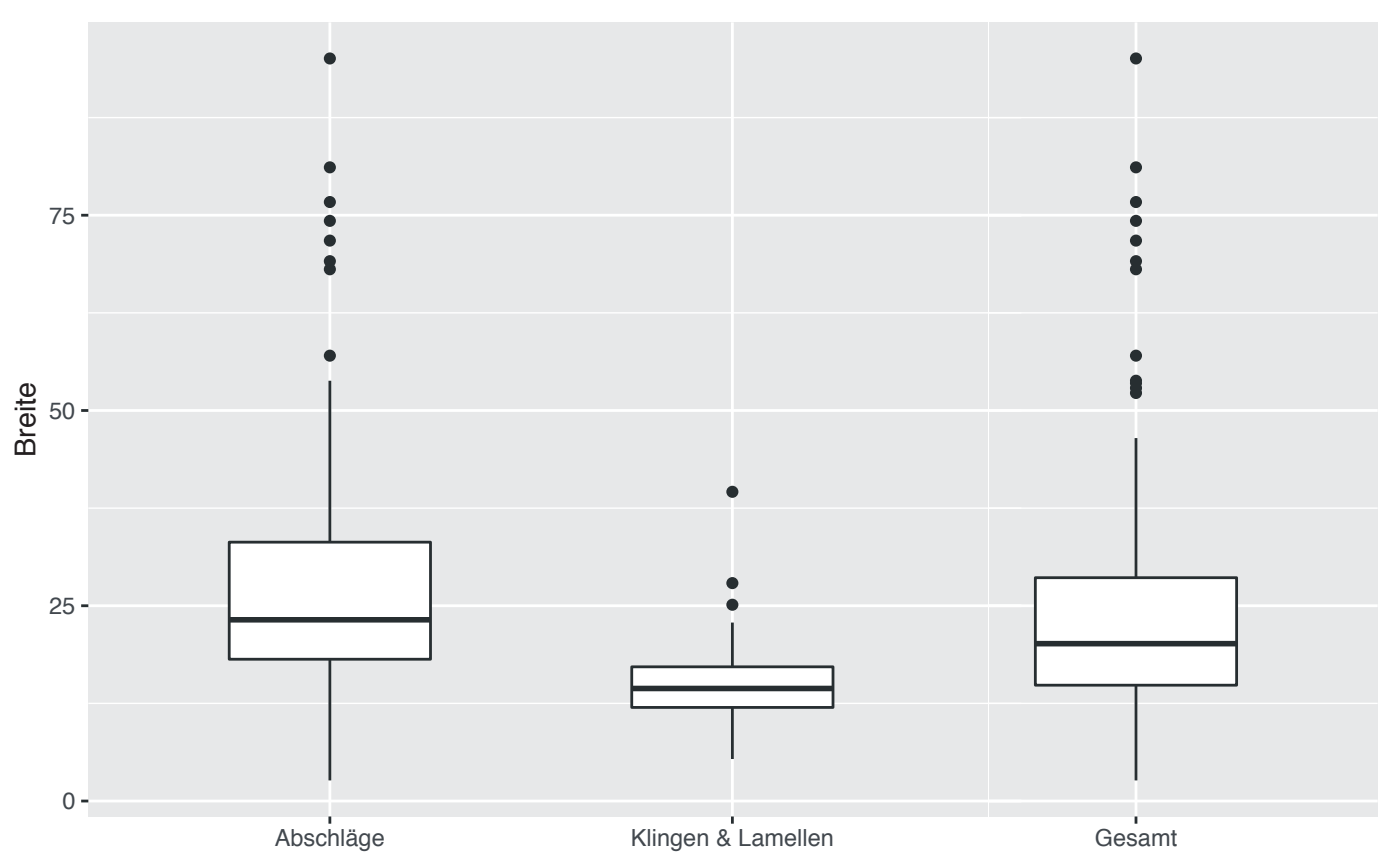

Abb. 9. Willendorf II, AH 8: Boxplot der Breite aller in der Breite vollständig erhaltenen Abschläge, Klingen und Lamellen sowie der Abschläge, Klingen und Lamellen gemeinsam. Alle Angaben in Millimeter.

\begin{tabular}{|l|c|c|c|c|c|c|c|c|c|c|c|c|}
\hline Artefaktklasse AH8 & \multicolumn{2}{|c|}{$0 \%$} & \multicolumn{2}{c|}{$1-33 \%$} & \multicolumn{2}{c|}{$33-66 \%$} & \multicolumn{2}{|c|}{$\mathbf{6 6 - 9 9} \%$} & \multicolumn{2}{c|}{$100 \%$} & \multicolumn{2}{c|}{ Gesamt } \\
\hline & $\mathrm{n}$ & $\%$ & $\mathrm{n}$ & $\%$ & $\mathrm{n}$ & $\%$ & $\mathrm{n}$ & $\%$ & $\mathrm{n}$ & $\%$ & $\mathrm{n}$ & $\%$ \\
\hline Klinge & 31 & $\mathbf{7 2 , 1} \%$ & 7 & $16,3 \%$ & 4 & $9,3 \%$ & - & - & 1 & $2,3 \%$ & 43 & $100 \%$ \\
\hline Lamelle & 8 & $\mathbf{6 6 , 7} \%$ & 3 & $25 \%$ & 1 & $8,3 \%$ & - & - & - & - & 12 & $100 \%$ \\
\hline Abschlag & 45 & $37,8 \%$ & 36 & $30,3 \%$ & 17 & $14,3 \%$ & 13 & $10,9 \%$ & 8 & $6,7 \%$ & 119 & $100 \%$ \\
\hline Kernscheibe & 4 & $\mathbf{6 6 , 7} \%$ & 1 & $16,7 \%$ & 1 & $16,7 \%$ & - & - & - & - & $\mathbf{6}$ & $100 \%$ \\
\hline Gesamt & $\mathbf{8 8}$ & $\mathbf{4 8 , 9} \%$ & $\mathbf{4 7}$ & $\mathbf{2 6 , 1} \%$ & $\mathbf{2 3}$ & $\mathbf{1 2 , 8} \%$ & 13 & $\mathbf{7 , 2} \%$ & $\mathbf{9}$ & $\mathbf{5} \%$ & 180 & $100 \%$ \\
\hline
\end{tabular}

Tab. 38. Willendorf II, AH 8: Anteile an natürlichen Oberflächen bei allen Grundformen.

Bei 9,7 \% der Abschläge sind die komplette Dorsalfläche und der Schlagflächenrest mit natürlichen Oberflächen bedeckt. Diese Primärabschläge, sogenannte entames im Sinne von Marie-Louise Inizan et al., ${ }^{143}$ stammen vom Beginn des Kernabbaus. 3,9 \% der Abschläge besitzen einen facettierten Schlagflächenrest.

Insgesamt weisen mit 35,4\% die meisten vollständig oder proximal erhaltenen Grundformen einen unregelmäBigen Schlagflächenrest auf (Tab. 42). Bei den Schlagflächenresten der Klingen und Lamellen tritt hauptsächlich die ovale Form auf. Bei den Abschlägen dominieren die Stücke

143 InIZAN et al. 1999. mit unregelmäßigem Schlagflächenrest. Danach folgen Abschläge mit ovalem Schlagflächenrest.

\subsubsection{Schlagtechnische Charakteristika}

Der Bulbus tritt mit 88,9 \% als häufigstes Merkmal bei den verschiedenen Grundformen in Erscheinung. Die Lippe stellt das zweithäufigste Merkmal dar, allerdings weisen die Abschläge dieses Merkmal wesentlich geringfügiger als die Klingen und Lamellen auf (Chi ${ }^{2}$-Test, $\mathrm{p}=0,0041$ ) (Tab. 43). 42,4\% der Grundformen weisen eine Schlagnarbe auf (Tab. 44). Bei den Abschlägen besitzt knapp über die Hälfte der Stücke eine Schlagnarbe. Stücke mit Kegel treten auf, 


\begin{tabular}{|l|c|c|}
\hline AH 8 & Lamellen/Klingen & Abschläge \\
\hline Anzahl & 22 & 60 \\
\hline Mittelwert & 7,34 & 16,24 \\
\hline Stand. Abw. & 1,71 & 2,44 \\
\hline Varianz & 14,92 & 89,17 \\
\hline $\mathrm{F}=$ & \multicolumn{2}{|c|}{5,9784} \\
\hline $\mathrm{t}=$ & \multicolumn{2}{|c|}{$-4,2742$} \\
\hline$p=$ & \multicolumn{2}{|c|}{$<0,0001$} \\
\hline
\end{tabular}

Tab. 39. Willendorf II, AH 8: Vergleich der SchlagflächenrestBreiten (in $\mathrm{mm}$ ) zwischen allen in der Länge vollständig und proximal erhaltenen Lamellen bzw. Klingen sowie Abschlägen ( $t$-Test).

\begin{tabular}{|l|c|c|}
\hline AH 8 & Lamellen/Klingen & Abschläge \\
\hline Anzahl & 22 & 60 \\
\hline Mittelwert & 2,65 & 6,38 \\
\hline Stand. Abw. & 0,62 & 1,68 \\
\hline Varianz & 1,97 & 42,34 \\
\hline $\mathrm{F}=$ & \multicolumn{2}{|c|}{$\mathbf{2 1 , 5 2 9}$} \\
\hline $\mathrm{t}=$ & $\mathbf{2}$ \\
\hline$p=$ & \multicolumn{2}{|c|}{0,0535} \\
\hline
\end{tabular}

Tab. 40. Willendorf II, AH 8: Vergleich der SchlagflächenrestDicken (in $\mathrm{mm}$ ) zwischen allen in der Länge vollständig und proximal erhaltenen Lamellen bzw. Klingen sowie Abschlägen ( $t-T e s t)$.

\begin{tabular}{|c|c|c|c|c|c|c|c|c|c|c|c|c|c|c|c|c|c|c|}
\hline \multirow[t]{2}{*}{ 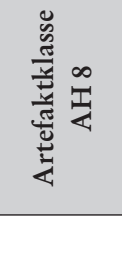 } & \multicolumn{2}{|c|}{ 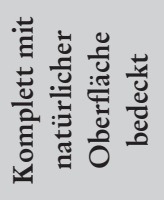 } & \multicolumn{2}{|r|}{ 苋 } & \multicolumn{2}{|c|}{ 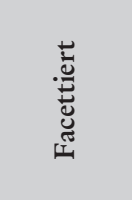 } & \multicolumn{2}{|r|}{$\frac{ \pm}{\frac{\pi}{U}}$} & \multicolumn{2}{|c|}{ 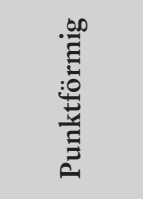 } & \multicolumn{2}{|c|}{ 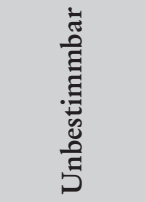 } & \multicolumn{2}{|c|}{$\stackrel{\rightleftarrows}{\Xi}$} & \multicolumn{2}{|c|}{ 芯 } & \multicolumn{2}{|r|}{ 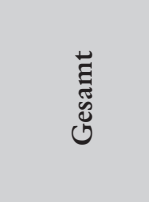 } \\
\hline & $\mathrm{n}$ & $\%$ & $\mathrm{n}$ & $\%$ & $\mathrm{n}$ & $\%$ & $\mathrm{n}$ & $\%$ & $\mathrm{n}$ & $\%$ & $\mathrm{n}$ & $\%$ & $\mathrm{n}$ & $\%$ & $\mathrm{n}$ & $\%$ & $\mathrm{n}$ & $\%$ \\
\hline Klinge & 2 & $9,5 \%$ & 1 & $4,8 \%$ & 1 & $4,8 \%$ & 16 & $76,2 \%$ & 1 & $4,8 \%$ & - & - & - & - & - & - & 21 & $100 \%$ \\
\hline Lamelle & - & - & - & - & - & - & 2 & $100 \%$ & - & - & - & - & - & - & - & - & 2 & $100 \%$ \\
\hline Abschlag & 31 & $40,8 \%$ & 5 & $6,6 \%$ & 3 & $3,9 \%$ & 30 & $39,5 \%$ & - & - & 2 & $2,6 \%$ & 3 & $3,9 \%$ & 2 & $2,6 \%$ & 76 & $100 \%$ \\
\hline Gesamt & 33 & $33,3 \%$ & 6 & $6,1 \%$ & 4 & $4 \%$ & 48 & $48,5 \%$ & 1 & $1 \%$ & 2 & $2 \%$ & 3 & $3 \%$ & 2 & $2 \%$ & 99 & $100 \%$ \\
\hline
\end{tabular}

Tab. 41. Willendorf II, AH 8: Art des Schlagflächenrestes bei allen in der Länge vollständig und proximal erhaltenen Grundformen.

\begin{tabular}{|c|c|c|c|c|c|c|c|c|c|c|c|c|c|c|c|c|c|c|}
\hline \multirow[t]{2}{*}{ 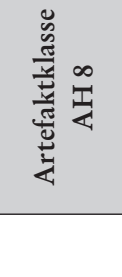 } & \multicolumn{2}{|c|}{ 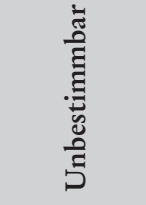 } & \multicolumn{2}{|c|}{ 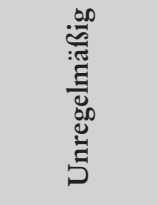 } & \multicolumn{2}{|c|}{ 壹 } & \multicolumn{2}{|r|}{$\overline{0}$} & \multicolumn{2}{|c|}{ } & \multicolumn{2}{|r|}{ 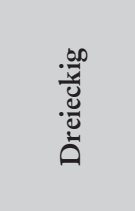 } & \multicolumn{2}{|c|}{ 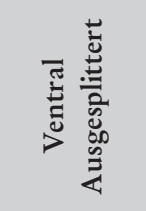 } & \multicolumn{2}{|r|}{ 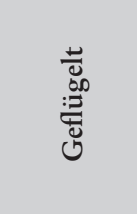 } & \multicolumn{2}{|r|}{ 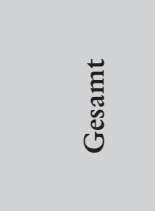 } \\
\hline & $\mathrm{n}$ & $\%$ & $\mathrm{n}$ & $\%$ & $\mathrm{n}$ & $\%$ & $\mathrm{n}$ & $\%$ & $\mathrm{n}$ & $\%$ & $\mathrm{n}$ & $\%$ & $\mathrm{n}$ & $\%$ & $\mathrm{n}$ & $\%$ & $\mathrm{n}$ & $\%$ \\
\hline Klinge & 1 & $4,8 \%$ & 1 & $4,8 \%$ & - & - & 14 & $66,7 \%$ & 1 & $4,8 \%$ & 3 & $14,3 \%$ & - & - & 1 & $4,8 \%$ & 21 & $100 \%$ \\
\hline Lamelle & - & - & - & - & - & - & 2 & $100 \%$ & - & - & - & - & - & - & - & - & 2 & $100 \%$ \\
\hline Abschlag & 7 & $9,2 \%$ & 34 & $44,7 \%$ & 2 & $2,6 \%$ & 16 & $21,1 \%$ & - & - & 4 & $5,3 \%$ & 8 & $10,5 \%$ & 5 & $6,6 \%$ & 76 & $100 \%$ \\
\hline Gesamt & 8 & $8,1 \%$ & 35 & $35,4 \%$ & 2 & $2 \%$ & 32 & $32,3 \%$ & 1 & $1 \%$ & 7 & $7,1 \%$ & 8 & $8,1 \%$ & 6 & $6,1 \%$ & 99 & $100 \%$ \\
\hline
\end{tabular}

Tab. 42. Willendorf II, AH 8: Form des Schlagflächenrestes bei allen in der Länge vollständig und proximal erhaltenen Grundformen. 


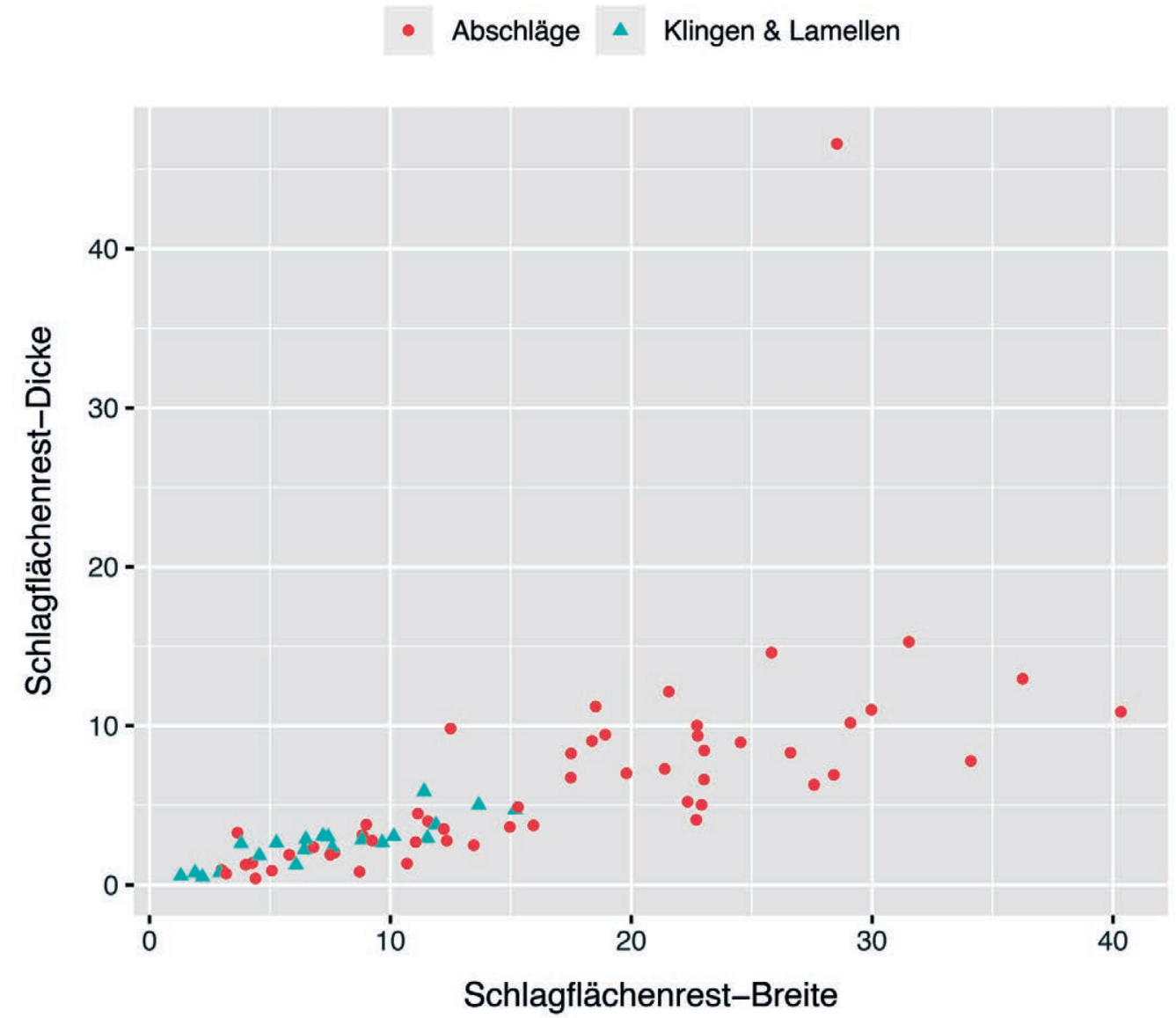

Abb. 10. Willendorf II, AH 8: Scatterplot der Breite und Dicke der Schlagflächenreste aller in der Länge vollständig oder proximal erhaltenen Abschläge sowie Lamellen und Klingen. Alle Angaben in Millimeter.

\begin{tabular}{|l|c|c|c|}
\hline Artefaktklasse AH 8 & Lippe & Keine Lippe & Gesamt \\
\hline Lamellen/Klingen & 21 & 38 & 59 \\
\hline Abschläge & 2 & 27 & 29 \\
\hline \multicolumn{3}{|c|}{ Chi $^{2}$-Test } \\
\hline F= & 1 \\
\hline Chi $^{2}=$ & $\mathbf{8 , 2 9 3 8}$ \\
\hline$p=$ & 0,003978 \\
\hline Fisher exact $p=$ & 0,0040851 \\
\hline
\end{tabular}

\begin{tabular}{|l|c|c|c|c|c|c|}
\hline $\begin{array}{l}\text { Artefaktklasse } \\
\text { AH 8 }\end{array}$ & \multicolumn{2}{|c|}{$\begin{array}{l}\text { Keine } \\
\text { Schlagnarbe }\end{array}$} & \multicolumn{2}{c|}{ Schlagnarbe } & \multicolumn{2}{c|}{ Gesamt } \\
\hline & $\mathrm{n}$ & $\%$ & $\mathrm{n}$ & $\%$ & $\mathrm{n}$ & $\%$ \\
\hline Lamellen/Klingen & 17 & $73,9 \%$ & 6 & $26,1 \%$ & $\mathbf{2 3}$ & $100 \%$ \\
\hline Abschlag & 40 & $52,6 \%$ & 36 & $47,4 \%$ & $\mathbf{7 6}$ & $100 \%$ \\
\hline Gesamt & $\mathbf{5 7}$ & $\mathbf{5 7 , 6} \%$ & $\mathbf{4 2}$ & $\mathbf{4 2 , 4 \%}$ & $\mathbf{9 9}$ & $100 \%$ \\
\hline
\end{tabular}

Tab. 44. Willendorf II, AH 8: Verteilung des Schlagmerkmals Schlagnarbe bei allen in der Länge vollständig und proximal erhaltenen Grundformen.

Tab. 43. Willendorf II, AH 8: Vergleich der Schlagflächenreste (SFR) mit Lippe und ohne Lippe zwischen allen vollständig und proximal erhaltenen Lamellen bzw. Klingen sowie Abschlägen (Chi'-Test). 
aber sind mit $2 \%$ selten (Tab. 45). Insgesamt dominieren mit 61,6 \% diejenigen Grundformen ohne dorsale Reduktion (Tab. 46). Bei 68,4 \% der Abschläge fehlt eine derartige Präparation der ehemaligen Schlagkante. 57,1 \% der Klingen und alle Lamellen weisen eine dorsale Reduktion auf. Die Messung der Abbauwinkel zeigte, dass die Winkel der Abschläge weiter streuen als bei den Klingen und Lamellen (Tab. 47). Durchschnittlich liegen die Klingen und Lamellen bei $81^{\circ}$ und die Abschläge bei $83^{\circ}$. Die Schlagflächenrest-Dicken der Klingen und Lamellen weisen einen Mittelwert von 2,65 mm auf, während die Abschläge mit 6,38 mm wesentlich massiver ausfallen ( $\mathrm{t}-$ Test, $\mathrm{t}=-2,6535, \mathrm{p}=0,0055$ ) (Tab. 40).

Für die Herstellung der Klingen und Lamellen zeichnet sich tendenziell der direkte, weiche, mineralische und/ oder der organische Schlag ab. Dies wird bestätigt durch die relativ dünnen und schmalen Schlagflächenreste und das häufige Auftreten von Lippen. Außerdem belegen alle Lamellen, aber nur 57,1 \% der Klingen mit der dorsalen Reduktion, eine Präparation der Schlagkante der Kerne. Die Schlagflächenrest-Dicken der Klingen und Lamellen mit dorsaler Reduktion liegen zwischen 0,5 und 3,8 mm, hingegen die der Klingen ohne dorsale Reduktion zwischen 2,7 und 5,9 mm (Wilcoxon-Mann-Whitney-Test, W=16, $\mathrm{p}=0.01247)$ (Tab. 48). Vermutlich musste durch das Nichtanbringen einer derartigen Präparation der Schlagpunkt sowohl beim direkten, weichen, mineralischen als auch beim direkten, organischen Schlag weiter nach innen versetzt werden, um einen Schlagunfall zu vermeiden. Es wäre auch anzunehmen, dass die Stücke ohne dorsale Reduktion und mit dickeren Schlagflächenresten tendenziell insgesamt länger, breiter und dicker sind, da die Schlagflächenrest-Dicke Einfluss auf die Grundformengröße nimmt. ${ }^{144}$ Allerdings konnte dies nicht nachgewiesen werden, da die geringe Anzahl dies nicht zulässt.

Bei den Abschlägen zeigen die zum Teil massiven Schlagflächenreste, das häufige Fehlen der dorsalen Reduktion und die Aussplitterungen bei einigen Stücken, dass diese in den meisten Fällen sehr wahrscheinlich der Präparation dienten und vorwiegend mit dem direkten, harten Schlag abgebaut worden sein dürften.

\subsubsection{Distalende}

Insgesamt machen die Grundformen mit Schlagunfällen distal (Angelbruch, Kernfuß, Stufenbruch) $50 \%$ aus (Tab. 49). Bei den wenigen vollständig oder distal erhaltenen Klingen

\begin{tabular}{|l|c|c|c|c|c|c|}
\hline Artefaktklasse AH 8 & \multicolumn{2}{|c|}{ Kein Kegel } & \multicolumn{2}{|c|}{ Kegel } & \multicolumn{2}{c|}{ Gesamt } \\
\hline & $\mathrm{n}$ & $\%$ & $\mathrm{n}$ & $\%$ & $\mathbf{n}$ & $\%$ \\
\hline Lamellen/Klingen & 22 & $95,7 \%$ & 1 & $4,3 \%$ & $\mathbf{2 3}$ & $100 \%$ \\
\hline Abschlag & 75 & $98,7 \%$ & 1 & $1,3 \%$ & $\mathbf{7 6}$ & $100 \%$ \\
\hline Gesamt & $\mathbf{9 7}$ & $\mathbf{9 8} \%$ & $\mathbf{2}$ & $\mathbf{2 \%}$ & $\mathbf{9 9}$ & $100 \%$ \\
\hline
\end{tabular}

Tab. 45. Willendorf II, AH 8: Verteilung des Schlagmerkmals Hertz'scher Kegel bei allen in der Länge vollständig und proximal erhaltenen Grundformen.

und Lamellen dominieren in der Seitenansicht diejenigen mit spitz zulaufenden Enden. 23,1 \% der Klingen enden in einem Federbruch. Von den drei Lamellen weist eine einen Federbruch auf. Bei den Klingen finden sich allerdings auch noch jeweils zwei mit Angelbruch und Kernfuß.

Bei den Abschlägen liegt eine wesentlich höhere Stückzahl vor und mit 34,7\% enden die meisten in einem Angelbruch. Am zweithäufigsten treten mit $26,4 \%$ in der Seitenansicht spitz zulaufende Abschläge auf. Abschläge mit Enden typisch für Federbrüche wurden bei 18,1 \% verzeichnet. 16,7 \% der Abschläge haben einen Kernfuß. Der insgesamt hohe Anteil an Schlagunfällen lässt vermuten, dass neben den schlagtechnischen Faktoren, die vermehrt zu Abschlägen mit Angelbrüchen, Stufenbrüchen oder Kernfußabschlägen führten, auch die Qualität der verwendeten Rohmaterialien eine Rolle gespielt haben dürften.

\subsubsection{Orientierung der dorsalen Negative}

Bei den Klingen und vor allem den Lamellen überwiegt der Anteil an solchen mit gleichgerichteten dorsalen Negativen (Tab. 50). Auch bei den Abschlägen dominieren diejenigen mit gleichgerichteten Negativen. 19,6 \% der Klingen und $24 \%$ der Abschläge weisen gleichgerichtete und quer verlaufende Negative auf. Hierfür dürfte einerseits das Anlegen von Kernkantenprodukten und andererseits die Präparation der Lateralflächen verantwortlich sein. Einige Abschläge und Klingen mit bidirektionalen Negativen wurden außerdem festgestellt. Bei sechs Abschlägen wurde dorsal ein Teil einer alten Ventralfläche festgestellt und aufgrund dieser Tatsache und anderer Charakteristika, wie Aussplitterungen an beiden Enden und starker Ausprägung der Wallnerlinien, dürften diese Abfälle von Ausgesplitterten Stücken darstellen (siehe auch Abschnitt 7.3.3.1). 


\begin{tabular}{|l|c|c|c|c|c|c|}
\hline & Lamellen/Klingen & \multicolumn{2}{|c|}{ Abschlag } & \multicolumn{2}{c|}{ Gesamt } \\
\hline & $\mathrm{n}$ & $\%$ & $\mathrm{n}$ & $\%$ & $\mathrm{n}$ & $\%$ \\
\hline Keine dorsale Reduktion & 9 & $39,1 \%$ & 52 & $68,4 \%$ & $\mathbf{6 1}$ & $\mathbf{6 1 , 6} \%$ \\
\hline Abrasion & 3 & $13 \%$ & 3 & $3,9 \%$ & $\mathbf{6}$ & $\mathbf{6 , 1} \%$ \\
\hline Spitz zulaufende Negative & 1 & $4,3 \%$ & 16 & $21,1 \%$ & 17 & $17,2 \%$ \\
\hline Negative mit Angelbrüchen und Abrasion & 9 & $39,1 \%$ & 5 & $6,6 \%$ & 14 & $14,1 \%$ \\
\hline Spitz zulaufende Negative und Abrasion & 1 & $4,3 \%$ & - & - & 1 & $1 \%$ \\
\hline Gesamt & $\mathbf{2 3}$ & $100 \%$ & 76 & $100 \%$ & $\mathbf{9 9}$ & $100 \%$ \\
\hline
\end{tabular}

Tab. 46. Willendorf II, AH 8: Dorsale Reduktion bei allen in der Länge vollständig und proximal erhaltenen Grundformen.

\begin{tabular}{|l|c|c|}
\hline AH 8 & Lamellen/Klingen & Abschläge \\
\hline Anzahl & 23 & 76 \\
\hline Min & 79 & 79 \\
\hline Max & 84 & 106 \\
\hline Mittelwert & 81,3 & 83,18 \\
\hline Stand. Abw. & 1,15 & 3,88 \\
\hline Median & 81 & 82 \\
\hline
\end{tabular}

\begin{tabular}{|l|c|c|}
\hline AH 8 & Dorsale Reduktion & Keine Dorsale Reduktion \\
\hline Anzahl & 14 & 8 \\
\hline Mittelwert & 2,04 & 3,71 \\
\hline Stand. Abw. & 1,09 & 1,29 \\
\hline Varianz & 1,19 & 1,68 \\
\hline F= & & 1,4137 \\
\hline W= & 16 \\
\hline$p=$ & 0,01247 \\
\hline
\end{tabular}

Tab. 47. Willendorf II, AH 8: Deskriptive Statistik des Abschlagwinkels (in Grad) aller in der Länge vollständig und proximal

Tab. 48. Willendorf II, AH 8: Vergleich der SchlagflächenrestDicken (in mm) zwischen aller in der Länge vollständig und proximal erhaltenen Klingen und Lamellen mit oder ohne dorsaler Reduktion (Wilcoxon-Mann-Whitney-Test).

\begin{tabular}{|l|c|c|c|c|c|c|c|c|c|c|c|c|}
\hline $\begin{array}{l}\text { Seitenansicht/ } \\
\text { Vorderansicht }\end{array}$ & \multicolumn{2}{|c|}{ Konkav } & \multicolumn{2}{|c|}{ Konvex } & \multicolumn{2}{|c|}{ Unregelmäßig } & \multicolumn{2}{|c|}{ Spitz zulaufend } & \multicolumn{2}{c|}{ Gerade } & \multicolumn{3}{c|}{ Gesamt } \\
\hline & $\mathrm{n}$ & $\%$ & $\mathrm{n}$ & $\%$ & $\mathrm{n}$ & $\%$ & $\mathrm{n}$ & $\%$ & $\mathrm{n}$ & $\%$ & $\mathrm{n}$ & $\%$ \\
\hline Angelbruch & - & - & 13 & $30,2 \%$ & 1 & $16,7 \%$ & 2 & $16,7 \%$ & 11 & $42,3 \%$ & 27 & $30,7 \%$ \\
\hline Kernfuß & - & - & 7 & $16,3 \%$ & 1 & $16,7 \%$ & 1 & $8,3 \%$ & 5 & $19,2 \%$ & 14 & $15,9 \%$ \\
\hline Spitz zulaufend & 1 & $100 \%$ & 13 & $30,3 \%$ & 3 & $50 \%$ & 7 & $58,3 \%$ & 5 & $19,2 \%$ & 29 & $33 \%$ \\
\hline Gerundet & - & - & 9 & $20,9 \%$ & - & - & 2 & $16,7 \%$ & 4 & $15,4 \%$ & 15 & $17 \%$ \\
\hline Stufenbruch & - & - & 1 & $2,3 \%$ & 1 & $16,7 \%$ & - & - & 1 & $3,8 \%$ & 3 & $3,4 \%$ \\
\hline Gesamt & 1 & $100 \%$ & 43 & $100 \%$ & $\mathbf{6}$ & $100 \%$ & 12 & $100 \%$ & $\mathbf{2 6}$ & $100 \%$ & $\mathbf{8 8}$ & $100 \%$ \\
\hline
\end{tabular}

Tab. 49. Willendorf II, AH 8: Form des Distalendes bei allen in der Länge vollständig oder distal erhaltenen Grundformen. 

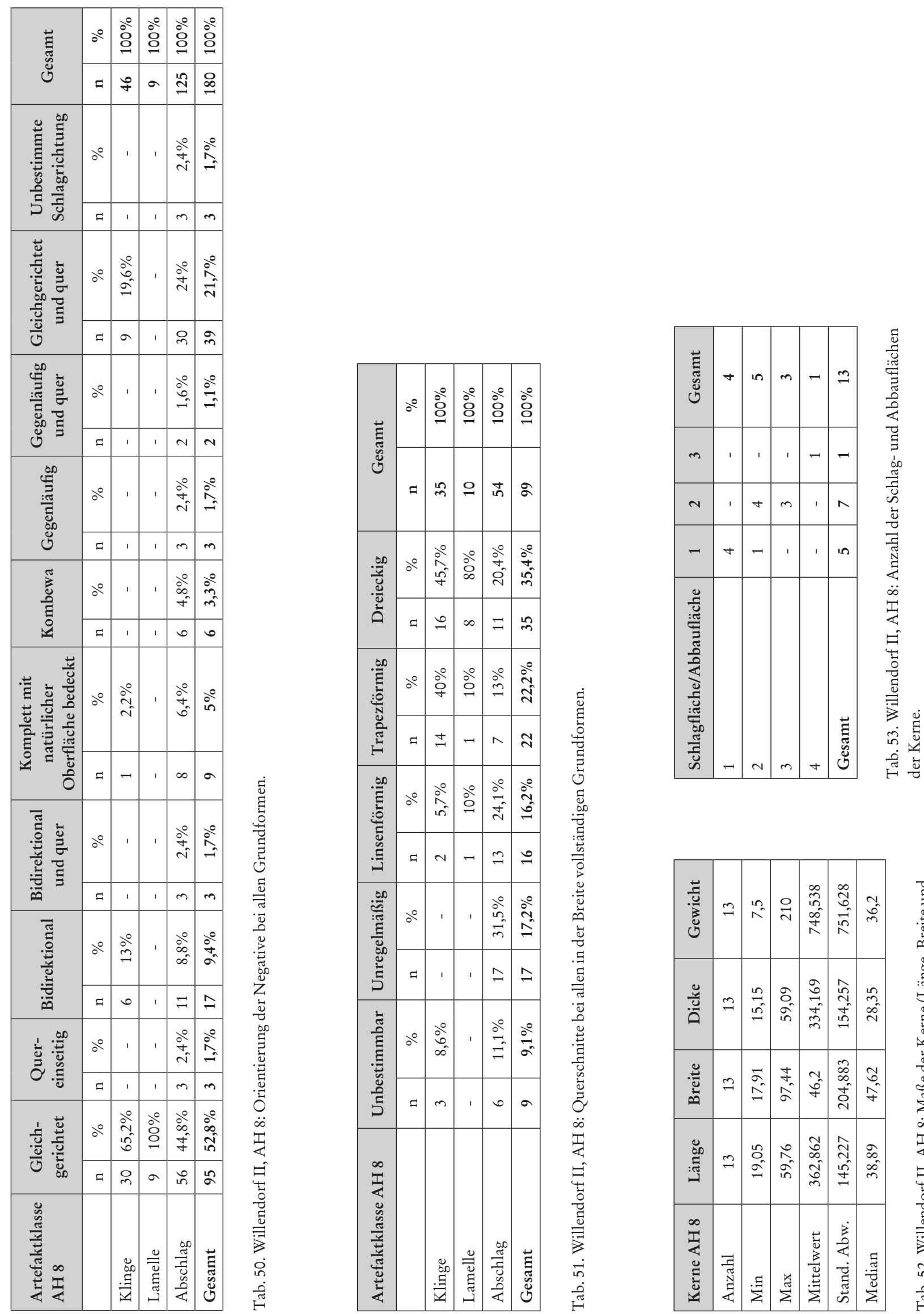

\begin{tabular}{|c|c|c|c|c|c|c|}
\hline 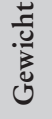 & $\stackrel{m}{\longrightarrow}$ & $\mathbb{1}^{n}$ & $\stackrel{\circ}{v}$ & 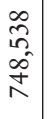 & 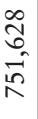 & 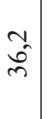 \\
\hline مُ & $\Rightarrow$ & $\stackrel{n}{n}$ & $\begin{array}{l}\text { ô } \\
\text { on }\end{array}$ & $\begin{array}{l}\vec{b} \\
\overrightarrow{0} \\
\tilde{c} \\
m\end{array}$ & 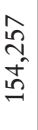 & 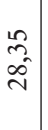 \\
\hline 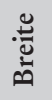 & 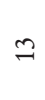 & $\begin{array}{l}\vec{\sigma} \\
\hat{\approx}\end{array}$ & $\begin{array}{l}\frac{+}{2} \\
\stackrel{2}{\sigma}\end{array}$ & $\begin{array}{l}\text { f̌ } \\
\text { fo }\end{array}$ & 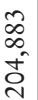 & $\begin{array}{l}\tilde{b} \\
\tilde{f}\end{array}$ \\
\hline 品 & 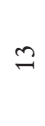 & 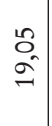 & $\begin{array}{l}\stackrel{2}{2} \\
\text { مे }\end{array}$ & $\begin{array}{l}0 \\
0 \\
0 \\
0 \\
\text { d }\end{array}$ & \begin{tabular}{l} 
Iิ \\
fo \\
\multirow{f}{*}{}
\end{tabular} & $\begin{array}{l}\sigma_{0} \\
\alpha^{\infty} \\
m\end{array}$ \\
\hline 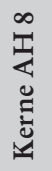 & 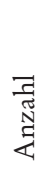 & $\dot{\Xi}$ & $\stackrel{\pi}{\Sigma}^{*}$ & 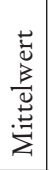 & 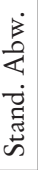 & 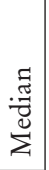 \\
\hline
\end{tabular}




\subsubsection{Querschnitte}

Bei Klingen und Lamellen sind dreieckige Querschnitte am häufigsten, danach folgen trapezförmige (Tab. 51). Bei den Abschlägen machen die Stücke mit unregelmäßigem Querschnitt mit 31,5\% den Hauptanteil aus. Bei der Herstellung der Klingen und Lamellen war demnach ein regelmäßig organisiertes Gratmuster gegeben bzw. erforderlich. Bei den Abschlägen hingegen dürfte ein regelmäßiges Gratmuster nicht notwendig gewesen sein, weil diese vermutlich keine Zielprodukte darstellten, sondern der Präparation dienten.

\subsubsection{Kerne}

13 Kerne liegen vor und umfassen insgesamt 5,1 \% des Inventars (Tab. 12). Zwei Kerntrümmer finden sich außerdem in AH 8, wurden aber bei der Auswertung der Kerne nicht berücksichtigt. Bis auf einen Kern weisen alle Kerne, die sehr intensiv abgebaut und daher vornehmlich wegen der zu kleinen Dimension für den weiteren Abbau verworfen wurden, ausschließlich Abschlagnegative im Verwerfungsstadium auf. Bei der einzigen Ausnahme wurden Lamellennegative festgestellt, doch beim letzten Abhub handelt es sich um das Negativ eines Abschlags mit Angelbruch.

\subsubsection{Ausgangsformen}

Bei vier Kernen war es aufgrund des starken Abbaus nicht mehr möglich zu ermitteln, welche Ausgangsform verwendet wurde. Für fünf der Kerne wurde ein Geröll als Ausgangsform verwendet. Diese dürften von den direkt an der Fundstelle gelegenen Donauschottern aufgesammelt und vermutlich ohne jegliches Vortesten in die Fundstelle eingebracht und verarbeitet worden sein. Bei drei der Kerne stellt die Ausgangsform ein größerer Abschlag dar. Gerade bei den beiden Kernen aus Serpentinit und Quarz wurde jeweils ein Abschlag als Kern genutzt. Es wird angenommen, dass bei diesen grobkörnigen Rohmaterialien zuerst überprüft wurde, inwieweit eine effiziente Herstellung von Grundformen durchgeführt werden kann. In einem Fall wurde ein Frostaussprung aus Kieselkalk als Kern herangezogen.

\subsubsection{Maße}

Die Kerne sind vorwiegend klein (Tab. 52). In einigen Fällen hängt dies mit der geringen Größe des verwendeten Ausgangsmaterials zusammen, aber vermehrt mit dem intensiven Kernabbau. Drei Kerne stechen mit großen Ausmaßen und vor allem durch ihr Gewicht mit ca. $200 \mathrm{~g}$ hervor. Bei zwei dieser Kerne trugen die Rohmaterialqualität kombiniert mit Angelbrüchen und beim dritten Angelbrüche dazu bei, dass nicht weiter abgebaut werden konnte.

\subsubsection{Schlag- und Abbaufläche}

Die Anzahl der Schlag- bzw. Abbauflächen lässt Aussagen zum Ablauf des Kernabbaus zu. Vier der Kerne besitzen eine einzige Schlagfläche und dazu eine Abbaufläche (Tab. 53). Fünf Kerne verfügen über zwei Schlagflächen. Vier dieser Kerne weisen auch eine zweite Abbaufläche auf und die Schlagflächen liegen jeweils rechtwinkelig zueinander. Ein Kern besitzt zu zwei gegenüberliegenden Schlagflächen eine Abbaufläche. Allerdings dürfte auch bei diesem Kern ein unidirektionaler und kein bidirektionaler Abbau erfolgt sein, da die Schlagflächen nicht, wie für den bidirektionalen Abbau typisch, alternierend genutzt wurden, sondern versetzt zueinander angelegt wurden. ${ }^{145}$ Drei Kerne haben drei Schlagflächen und zwei Abbauflächen. In einem Fall liegen die Schlagflächen rechtwinkelig zueinander, während die Schlagflächen der anderen beiden Restkerne einander gegenüberliegen. Aber wiederum zeigt sich, dass die Ausrichtung der einen zur anderen Schlagfläche versetzt angelegt wurde. Lediglich bei einem Kern wurden sogar vier zueinander rechtwinkelig liegende Schlagflächen angelegt und dazugehörig drei Abbauflächen genutzt.

Die Untersuchung der Schlagflächenart liefert Informationen darüber, wie viel in die Präparation der Kerne investiert wurde. Wie auch schon anhand der Schlagflächenreste der Grundformen festgestellt wurde, zeigt sich, dass die Mehrheit ( $\mathrm{n}=11)$ der Schlagflächen glatt ist. Bei den übrigen zwei Kernen liegt eine Schlagfläche bedeckt mit natürlicher Oberfläche vor. Offenbar stellte in diesen Fällen die natürliche Oberfläche kein Problem für den Abbau von Zielprodukten dar und konnte daher als Schlagfläche genutzt werden. Bei mehr als $75 \%$ der Kerne wurde eine Präparation der Schlagflächenkanten auf die Abbaufläche (dorsale Reduktion) festgestellt.

\subsubsection{Abbaurichtung}

Unter vorwiegender Berücksichtigung der Hauptabbaufläche, d. h. jener Abbaufläche mit dem Negativ der letzten abgebauten Grundform, konnte nachgewiesen werden, dass alle Kerne parallel verlaufende Negative aufweisen. Der Abbau dürfte sich in all seinen Phasen grundsätzlich, wie auch schon durch die Grundformen belegt, unidirektional gestaltet haben. Bei drei Kernen wurden, wie bei den Schlagund Abbauflächen bereits angesprochen, Abbauflächen mit Negativen von zwei gegenüberliegenden Schlagflächen festgestellt. Allerdings würde ein bidirektionaler Abbau voraussetzen, dass beiden Schlagflächen dieselbe Funktion bei der Gewinnung der Zielprodukte zukommt, indem eine alternierende Nutzung vorliegt und der Kern um jeweils $180^{\circ}$ gedreht wurde. ${ }^{146} \mathrm{Im}$ vorliegenden Szenario wurde jedoch

145 Siehe Moreau 2009, 73.

146 Moreau 2009, 73. 
die eine Schlagfläche versetzt zur anderen angelegt und dies dürfte dazu gedient haben, die Abbauflächenkonvexität wiederherzustellen.

\subsubsection{Lateralfläche, Rücken, Kernfuß}

Die Lateralflächen werden am häufigsten durch Negative gebildet, die zu einer anderen Abbaufläche gehören. Bei drei der Kerne bestehen die Lateralflächen aus natürlichen Oberflächen und bei weiteren drei aus einer Kluft. Somit ist in keinem Fall eine eindeutige laterale Präparation nachgewiesen. Allerdings könnte eine ursprüngliche Präparation aufgrund des starken Kernabbaus bereits entfernt worden sein.

Mehr als die Hälfte der verworfenen Kerne besitzen entweder einen kortikalen Rücken oder eine Kluftfläche bzw. in einem Fall eine Frostaussprungsfläche. Bei zwei der Kerne besteht der Rücken aus der ehemaligen Ventralfläche des Ausgangsabschlags. Bei weiteren zwei ist kein Rücken vorhanden, weil sich eine andere Schlagfläche stattdessen dort befindet. Bei den übrigen zwei wird der Rücken von Negativen einer anderen Abbaufläche gebildet, so dass über die Ausprägung des Rückens keine Aussage getroffen werden kann. Kein Kern weist eine eindeutige Präparation des Rückens auf. Doch aufgrund der Überprägung durch weitere Schlag- und Abbauflächen, womit die intensive Kernausnutzung angezeigt wird, kann eine anfängliche Präparation nicht ausgeschlossen werden.

Der Kernfuß besteht bei fünf Kernen aus natürlichen Oberflächen. Bei vier Kernen befindet sich eine andere Abbaufläche an der Stelle des Kernfußes. Drei Kerne weisen eine zweite Schlagfläche auf und ein Kern wurde präpariert. Die Präparation des Kernfußes wird vorgenommen, um die longitudinale Konvexität der Abbaufläche zu erhalten. ${ }^{147}$ Es kann davon ausgegangen werden, dass auch das Anlegen der zweiten Schlagfläche am Kernfuß eine Art der Präparation zur Erhaltung der Abbauflächenkonvexität darstellt.

\subsubsection{Letzte Kernabbauphase}

Die Kerne informieren über die letzte Abbauphase, daher können Aussagen darüber getroffen werden, ab wann ein Kern nicht mehr als abbaufähig galt und verworfen wurde. Von den Hauptabbauflächen wurden die Abbauwinkel zu den Schlagflächen ermittelt. Es zeigte sich, dass sechs der Kerne vom Abbauwinkel her zwischen 79 und $84^{\circ}$ liegen (Tab. 54). Dieser Bereich entspricht durchaus den Abbauwinkeln der Klingen und Lamellen und der Mehrheit der Abschläge. Die übrigen Kerne weisen höhere Abbauwinkel auf. Der Maximalwert liegt bei $94^{\circ}$. Je höher die Winkel, desto höher die Wahrscheinlichkeit für Schlagunfälle, die dann zum Verwerfen der Kerne führen können (siehe
7.3.2.7). Die Längen der letztabgebauten Abschläge fielen gering aus (Tab. 55). Lediglich ein Negativ ist länger als $20 \mathrm{~mm}$. Tatsächlich dürften nicht kurze, kleine Abschläge angestrebt worden sein, sondern eine verfrühte Endigung in einem Angelbruch als Schlagunfall dürfte diese geringen Längenwerte verursacht haben.

\begin{tabular}{|l|c|}
\hline AH 8 & Kerne \\
\hline Anzahl & 13 \\
\hline Min & 79 \\
\hline Max & 94 \\
\hline Mittelwert & 85,23 \\
\hline Stand. Abw. & 5,07 \\
\hline Median & 85 \\
\hline
\end{tabular}

Tab. 54. Willendorf II, AH 8: Abbauwinkel der Kerne (in Grad).

\begin{tabular}{|l|c|}
\hline AH 8 & Kerne \\
\hline Anzahl & 13 \\
\hline Min & 3,43 \\
\hline Max & 26,59 \\
\hline Mittelwert & 9,43 \\
\hline Stand. Abw. & 7,42 \\
\hline Median & 5,2 \\
\hline
\end{tabular}

Tab. 55. Willendorf II, AH 8: Länge (in mm) bei allen letztabgebauten Grundformen der Kerne.

\subsubsection{Verwerfungsgründe}

In den meisten Fällen waren entweder die Dimension oder Angelbrüche bzw. eine Kombination aus den beiden verantwortlich für die Aufgabe des Kerns. Die Rohmaterialqualität bildet ebenso einen wichtigen mitwirkenden Faktor. Angelbrüche sind wahrscheinlich ein Resultat von schlechter Rohmaterialqualität und/oder hohen Abbauwinkeln. Zum Beispiel sind in dem Inventar Kerne mit hohen Abbauwinkeln zumeist wegen dem vermehrten Auftreten von Angelbrüchen verworfen worden. Die letzten Negative auf der Hauptabbaufläche waren bei allen Kernen Abschlagnegative, die zum Großteil in einem Angelbruch endeten, und waren daher wahrscheinlich mitverantwortlich für die Verwerfung des Kerns.

Im Detail war bei drei der vier Kerne mit jeweils einer Schlag- und Abbaufläche die Dimension ein entscheidender Verwerfungsgrund. Der vierte Kern wies eine Kombination 
aus schlechter Rohmaterialqualität und Angelbrüchen auf. Bis auf einen Kern waren bei allen Kernen mit mehr als einer Schlagfläche Angelbrüche der Hauptgrund für die Verwerfung. Die Ausnahme stellt ein Kern mit jeweils zwei Schlag- und Abbauflächen dar. Er wurde wegen seiner Dimension und wahrscheinlich auch wegen der Rohmaterialqualität aufgegeben. Dies dürfte darauf hindeuten, dass eigentlich Kerne mit einer einzigen Schlag- und Abbaufläche für den unidirektionalen Abbau vorgesehen waren und erst beim Auftreten von Angelbrüchen, wenn die Dimension noch ausreichend war bzw. die Qualität des Rohmaterials es zuließ, weitere Schlag- und Abbauflächen angelegt wurden.

\subsubsection{Zusammenpassungen}

19 Grundformen aus AH 8 konnten zusammengepasst werden. Daher ergibt sich eine Zusammenpassungsrate von 9,3 \%. Neun Zusammenpassungskomplexe wurden gefunden (Tab. 56). Acht der Zusammenpassungskomplexe bestehen aus zwei Stücken und einer aus drei Stücken.

\begin{tabular}{|c|c|c|c|}
\hline 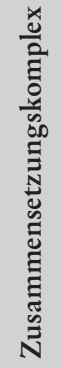 & 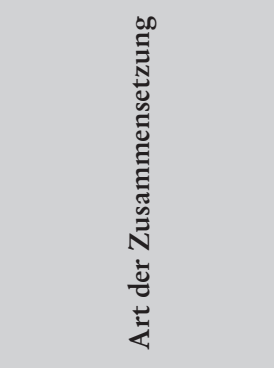 & 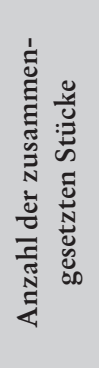 & 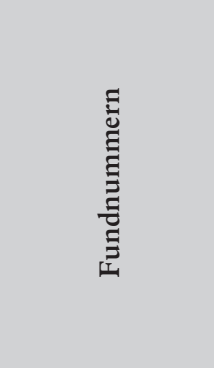 \\
\hline 002 & Aneinanderpassung & 2 & $\begin{array}{l}\text { WII-1993-99-1 } \\
\text { WII-1993-99-3 }\end{array}$ \\
\hline 015 & Aufeinanderpassung & 2 & $\begin{array}{c}\text { WII-1993-79-1 } \\
\text { WII-1993-83 }\end{array}$ \\
\hline 017 & Aneinanderpassung & 3 & $\begin{array}{l}\text { WII-1993-88-3 } \\
\text { WII-1993-88-5 } \\
\text { WII-1993-88-6 }\end{array}$ \\
\hline 018 & Anpassung - splinters & 2 & $\begin{array}{l}\text { WII-1993-64-1 } \\
\text { WII-1993-64-2 }\end{array}$ \\
\hline 019 & Anpassung - splinters & 2 & $\begin{array}{l}\text { WII-1993-50-1 } \\
\text { WII-1993-50-3 }\end{array}$ \\
\hline 020 & Aneinanderpassung & 2 & $\begin{array}{l}\text { WII-1993-125-1 } \\
\text { WII-1993-125-2 }\end{array}$ \\
\hline 022 & Aneinanderpassung & 2 & $\begin{array}{l}\text { WII-1993-90-11 } \\
\text { WII-1993-90-12 }\end{array}$ \\
\hline 023 & Aneinanderpassung & 2 & $\begin{array}{l}\text { WII-1993-126-2 } \\
\text { WII-1993-139-2 }\end{array}$ \\
\hline 025 & Einpassung & 2 & $\begin{array}{l}\text { WII-1993-117-1 } \\
\text { WII-1993-117-2 }\end{array}$ \\
\hline
\end{tabular}

Tab. 56. Willendorf II, AH 8: Zusammensetzungskomplexe der Grundformen und Werkzeuge.

\subsubsection{Zusammenpassungstypen}

Vorwiegend konnten Fragmente aneinandergepasst werden, denn von den insgesamt neun Zusammenpassungskomplexen handelt es sich bei fünf um Aneinanderpassungen. In zwei Fällen liegen Aufeinanderpassungen bzw. eigentlich vielmehr Anpassungen von Aussplitterungen, sogenannten splinters, ${ }^{148}$ an Ausgesplitterte Stücke vor. Außerdem gelangen eine Aufeinanderpassung und eine Einpassung. Daher wurde neben der Grundformenproduktion auch die Nutzung von Ausgesplitterten Stücken vor Ort nachgewiesen. Sehr wahrscheinlich wurden außerdem verschiedene Werkzeugformen direkt an der Fundstelle hergestellt, allerdings konnten dafür keine Belege gefunden werden.

Bei sechs der sieben am häufigsten vorkommenden Rohmaterialien konnten Stücke zusammengepasst werden. Die einzige Ausnahme stellt der baltische Feuerstein dar. Die meisten Zusammenpassungen wurden mit 47,4 \% unter den Quarziten gefunden. Die einzige vorliegende Aufeinanderpassung stellen jedoch zwei Grundformen aus Quarz dar.

Knapp $75 \%$ der Zusammenpassungen fanden sich bei den Abschlägen. Lediglich zwei Klingen- und zwei Lamellenfragmente konnten wieder zusammengesetzt werden. Außerdem konnte eine Einpassung eines Hitzetrümmers an einen der Abschläge vorgenommen werden.

Im Folgenden wird eine kurze Beschreibung der wichtigsten Zusammenpassungskomplexe gegeben:

Zusammenpassungskomplex 018: Ein Abfall eines Ausgesplitterten Stückes aus Quarzit konnte mit dem Ausgesplitterten Stück, von dem er stammte, wieder zusammengesetzt werden (Abb. 11). Das Ausgesplitterte Stück weist sowohl in der Längsachse als auch in der Querachse Aussplitterungen auf. Der Abfall des Ausgesplitterten Stückes splitterte von der Dorsalfläche des Ausgesplitterten Stückes ab.

Zusammenpassungskomplex 019: Hierbei handelt es sich ebenfalls um eine Aufeinander- bzw. Anpassung eines splinter aus Quarzit an ein Ausgesplittertes Stück (Abb. 12). Das Ausgesplitterte Stück weist an Längs- und Querachse Aussplitterungen auf. Der splinter weist dorsal Reste der Ventralfläche und des Schlagflächenrestes des Ausgesplitterten Stückes auf, von welchem er aussplitterte.

Zusammenpassungskomplex 015: Ein Abschlag aus Quarz wurde auf einen weiteren Abschlag aufgesetzt. Beide Abschläge sind vollständig erhalten. Ihre Distalenden sind gerundet. Beide Schlagflächenreste sind unregelmäßig und mit natürlichen Oberflächen bedeckt. Die Schlagflächenrest-Dicken liegen bei 14,6 mm und 11,2 mm. Außerdem wurden bei beiden Siretbrüche und deutlich ausgeprägte

148 Siehe Le Brun-Ricalens 2006. 


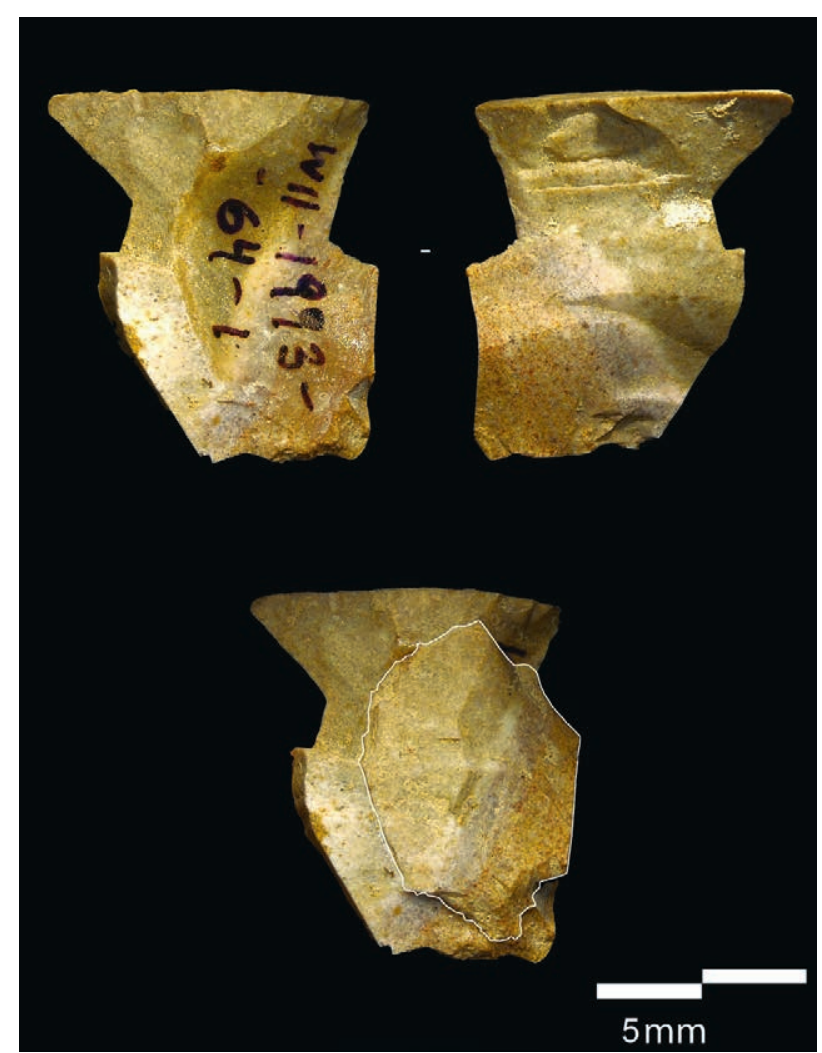

Abb. 11. Willendorf II, AH 8: Zusammenpassungskomplex 018.

Schlagpunkte festgestellt. Sinistrolateral sind beide Abschläge komplett mit natürlichen Oberflächen bedeckt. Die dorsalen Negative verlaufen in beiden Fällen gleichgerichtet. Aufgrund der starken Bedeckung mit natürlichen Oberflächen wird angenommen, dass beide Abschläge eher in einem frühen Abbaustadium hergestellt wurden. Verschiedenste Indikatoren wie Schlagpunkte, die massive Schlagflächenrest-Dicken und die Siretbrüche lassen darauf schließen, dass die Grundformen mit dem direkten, harten Schlag gelöst wurden. Zusätzlich weisen die dorsalen Negative auf einen unidirektionalen Kernabbau.

Die zusammengepassten Artefakte aus AH 8 weisen auf einen sicheren stratigrafischen Kontext. Sieben der neun Zusammenpassungen wurden innerhalb eines Sammelfundes festgestellt, womit die Stücke relativ nah zueinander lagen. Sowohl bei der Aufeinanderpassung als auch einer der Aneinanderpassungen liegen für alle zugehörigen Funde exakte Angaben zur Lage vor.

\subsubsection{Werkzeuge}

$12,2 \%$ von 189 Grundformen wurden modifiziert und bei $9 \%$ handelt es sich um Ausgesplitterte Stücke - somit

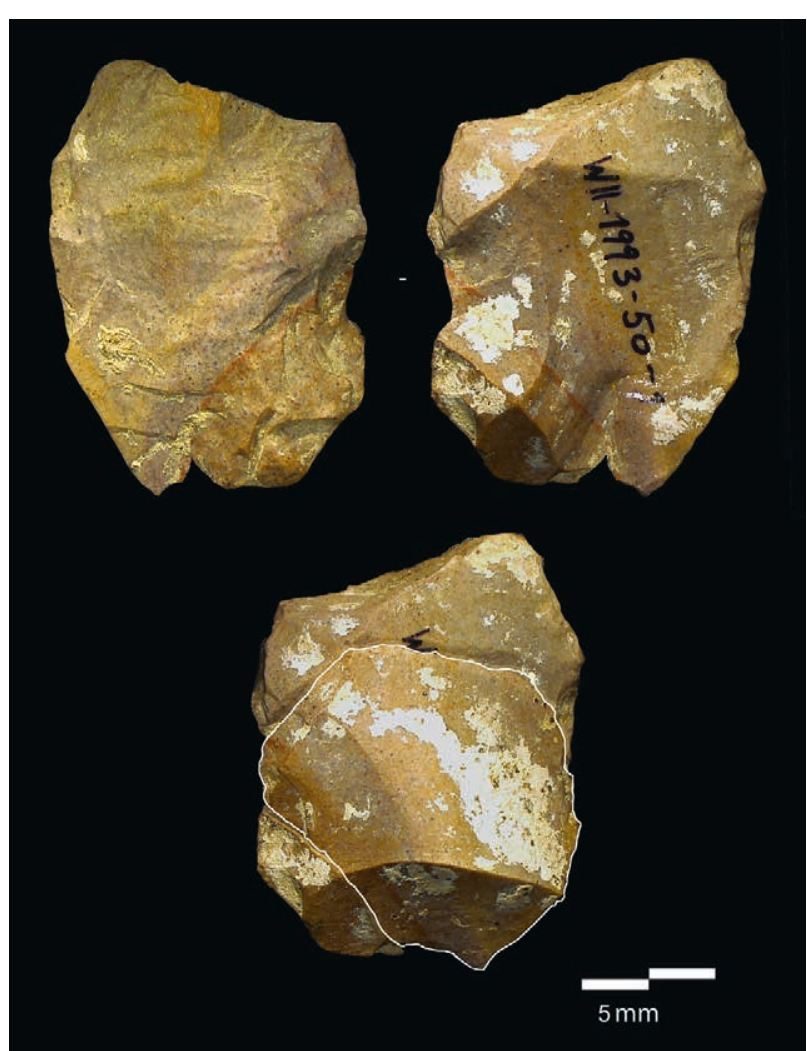

Abb. 12. Willendorf II, AH 8: Zusammenpassungskomplex 019.

machen die Werkzeuge 21,2 \% des Gesamtinventars aus. Die am häufigsten auftretenden Werkzeugtypen sind Ausgesplitterte Stücke und Kantenretuschen (Tab. 57). Der Großteil der Werkzeuge wurde an Abschlägen hergestellt. Bei 15 der 40 Werkzeuge wurden Klingen als Ausgangsformen herangezogen. Bei 14 der 17 Ausgesplitterten Stücke stellt die ehemalige Grundform einen Abschlag dar, während hingegen bei den Kantenretuschen Klingen bevorzugt als Ausgangsformen dienten. Es dürfte ein Trend dahingehend vorliegen, dass für bestimmte Werkzeugtypen wie Kantenretuschen und Spitzklingen gezielt Klingen aufgrund ihrer länglichen Form ausgewählt wurden und bei anderen Werkzeugtypen wie bei Ausgesplitterten Stücken die Morphologie nicht so wichtig erschienen sein dürfte. Nun folgt die Beschreibung der einzelnen Werkzeugtypen:

Ausgesplitterte Stücke: Ausgesplitterte Stücke haben typischerweise an ihren zwei gegenüberliegenden Enden Aussplitterungen, aber in seltenen Fällen - und auch im Inventar von AH 8 - treten Aussplitterungen in Längs- und Querachse und somit an allen vier Seiten auf. ${ }^{149}$

149 Hahn 1993, 248. - Le Brun-Ricalens 2006, 98. 


\begin{tabular}{|l|c|}
\hline Werkzeugtyp AH 8 & Gesamt \\
\hline Ausgesplittertes Stück & 17 \\
\hline Kantenretusche & 9 \\
\hline Stichel & 3 \\
\hline Ausgesplittertes Stück und Kantenretusche & 2 \\
\hline Endretusche & 2 \\
\hline Kratzer und Kantenretusche & 2 \\
\hline Spitzklinge & 2 \\
\hline Bohrer und Kantenretusche & 1 \\
\hline Kratzer & 1 \\
\hline Gezähntes Stück & 1 \\
\hline Gesamt & 40 \\
\hline
\end{tabular}

Tab. 57. Willendorf II, AH 8: Typologische Zusammensetzung der Werkzeuge.

Die meisten Ausgesplitterten Stücke bestehen aus Quarzit, gefolgt von jenen aus Jaspis. Die Länge der Stücke liegt zwischen 10,9 und $37,8 \mathrm{~mm}$, die Breite zwischen 10,9 und $32,8 \mathrm{~mm}$ und die Dicke zwischen 2,6 und 12,4 mm (Tab. 58).

\begin{tabular}{|l|c|c|c|c|}
\hline AH 8 & Länge & Breite & Dicke & Gewicht \\
\hline Anzahl & 17 & 17 & 17 & 17 \\
\hline Min & 10,92 & 10,93 & 2,6 & 0,5 \\
\hline Max & 37,8 & 32,84 & 12,4 & 8,6 \\
\hline Mittelwert & 23,23706 & 18,35529 & 6,06 & 2,811765 \\
\hline Stand. Abw. & 6,424213 & 5,083736 & 2,133257 & 2,051488 \\
\hline Median & 23,66 & 17,86 & 5,76 & 2,4 \\
\hline
\end{tabular}

Tab. 58. Willendorf II, AH 8: Maße (Länge, Breite und Dicke in $\mathrm{mm}$; Gewicht in g) aller Ausgesplitterten Stücke.

Im Allgemeinen werden Ausgesplitterte Stücke als Kerne und/oder Werkzeugformen, bei welchen die Modifikation nicht durch intentionelle Retusche, sondern durch den Gebrauch als Zwischenstücke für den indirekten, bipolaren Schlag entsteht, interpretiert. ${ }^{150}$ Die Nutzung einiger Ausgesplitterter Stücke als bipolare Kerne, um kleine, mehr oder weniger längliche Abschläge zu produzieren, wurde an einigen paläolithischen Fundstellen wie Barbera Caves (Italien), Buraca Grande, Buraca Escura und Salto do Boi (Portugal) nachgewiesen. ${ }^{151}$ Aber Ausgesplitterte Stücke können eben auch als Werkzeuge zum Spalten, Schneiden, Kerben, Bohren, Brechen etc. harter, organischer

150 Hahn 1993, 249. - Le Brun-Ricalens 2006, 101. 151 Aubry, Fontugne, Moura 1997. - D’Angelo, Mussi 2005.
Materialien verwendet werden. ${ }^{152} \mathrm{Zwar}$ wurden bisher keine Gebrauchsspurenanalysen an den Ausgesplitterten Stücken aus AH 8 durchgeführt, aber dennoch dürfte es sich eher um Werkzeugformen gehandelt haben, die unmittelbar vor Ort genutzt und weggeworfen wurden. Diese Annahme wird vor allem dadurch gestützt, dass zwei Ausgesplitterte Stücke jeweils mit einem von ihnen ausgesplitterten Abschlag zusammengepasst werden konnten und es sich bei diesen Abschlägen eher nicht um Zielprodukte handeln dürfte.

Es existieren im Inventar, wie schon angesprochen, insgesamt neun Abschläge, die als Abfälle von Ausgesplitterten Stücken, splinter, identifiziert werden konnten. Die Wallnerlinien dieser speziellen Abschläge sind oft sehr stark ausgeprägt und liegen sehr eng beieinander. Negative an Ausgesplitterten Stücken belegen, dass die Aussplitterungen sowohl dorsal als auch ventral auftreten können. ${ }^{153}$ Bei sechs der neun Abschläge existiert dorsal der Rest einer ehemaligen Ventralfläche, somit wurden diese Stücke eindeutig von der Ventralfläche ausgesplittert. Diese splinter sind oftmals kurz und invasiv und können zusätzlich entweder in einem Angelbruch, Stufenbruch oder als outrepassés enden. ${ }^{154}$ Tatsächlich zeigt sich, dass die Abfälle von Ausgesplitterten Stücken aus AH 8 eher kurz sind, vor allem auch im Vergleich zu den in Länge und Breite vollständig erhaltenen Abschlägen insgesamt (Abb. 13).

Ausgesplittertes Stück und Kantenretusche: Zwei der Ausgesplitterten Stücke weisen zusätzlich eine Kantenretusche auf (Abb. 14/WII-1993-140-1). Nachdem diese Artefakte als Kantenretuschen nicht mehr einsatzfähig erschienen, wurden sie in einer letzten Verwendungsphase noch als Ausgesplitterte Stücke herangezogen. Bei beiden handelt es sich um Quarzite, deren Kantenretusche in der Länge aufgrund des nachträglich erfolgten Gebrauchs als Ausgesplitterte Stücke nicht vollständig erhalten blieb. Vor allem in Bezug auf Länge und Breite (Abb. 15), aber auch Dicke und Gewicht betreffend, fallen die Werkzeugformen in das Spektrum der Ausgesplitterten Stücke ohne Kantenretusche. Bei einem der beiden Stücke handelt es sich um eine Klinge mit einer geraden, starken, dorsalen Retusche sinistrolateral, die 2,9 mm hoch ist. Bei dem anderen Stück diente ein Abschlag als Ausgangsform und die dextrolaterale, 2,4 $\mathrm{mm}$ hohe Retusche ist ebenfalls gerade und stark, aber ventral.

Einer der eben angesprochenen neun splinter weist eine Kantenretusche auf und splitterte daher von einer retuschierten Grundform ab.

152 Hahn 1993, 249. - Le Brun-Ricalens 2006, 101.

153 Le Brun-Ricalens 2006, 98.

154 Le BRun-Ricalens 2006, 98. 


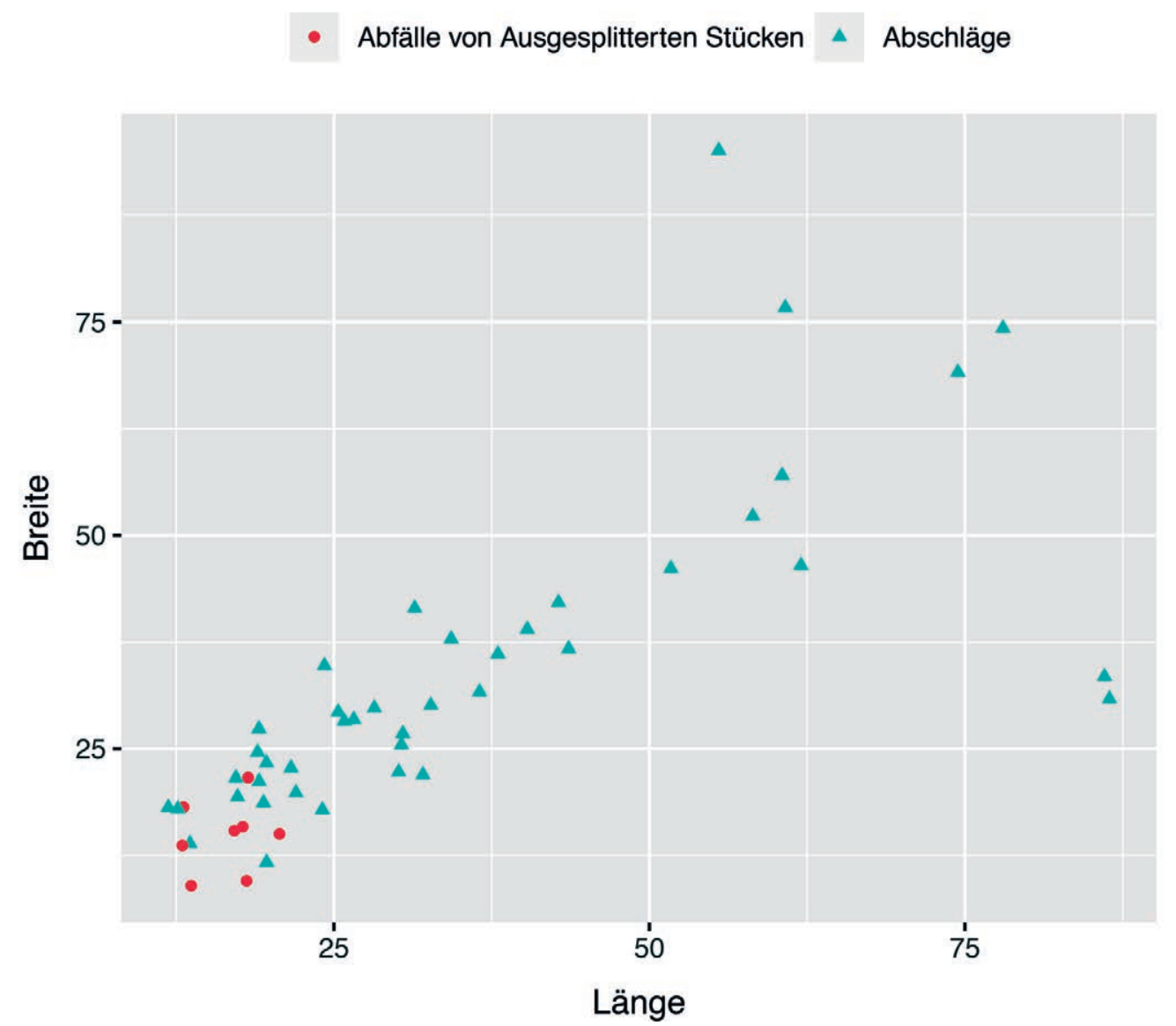

Abb. 13. Willendorf II, AH 8: Scatterplot der Länge und Breite der (in Länge und Breite vollständig erhaltenen) Abschläge und Abfälle von Ausgesplitterten Stücken. Alle Angaben in Millimeter.

Stichel: Alle drei Stichel wurden an Grundformen aus Quarzit hergestellt. Als Ausgangsformen dienten eine sekundäre Kernkantenlamelle und zwei Abschläge. Lediglich die Stichelbahnen an der sekundären Kernkantenlamelle sind vollständig erhalten (Abb. 14/WII-1993-49). Es wurden zwei Stichelbahnen an Bruch angelegt. Die eine Stichelbahn mit einer Länge von 15,3 mm und einer Breite von 2,1 $\mathrm{mm}$ wurde von proximal an der dextrolateralen Kante angesetzt, während die andere mit einer Länge von 7,9 mm und einer Breite von 1,4 mm distal an der sinistrolateralen Kante verläuft. Zusätzlich liegen ein Mehrschlagstichel und ein Stichel an Bruch vor.

Kantenretuschen: Insgesamt gibt es neun Kantenretuschen. Bei sieben Kantenretuschen stellten Klingen die Ausgangsformen dar, lediglich bei zweien wurden Abschläge herangezogen. Ein Drittel der Kantenretuschen ist in der Länge vollständig erhalten, darunter ausschließlich Kantenretuschen an Klingen. Bei einer dieser vollständig erhaltenen Kantenretuschen liegen an der dextrolateralen Kante zwei verschiedene Modifikationen vor - einerseits eine konvexe, starke Retusche distal mit einer Länge von 19,5 mm und Höhe von 2,4 $\mathrm{mm}$ und anderseits eine gerade, starke, alternierende Retusche, die 24,3 mm lang und $2 \mathrm{~mm}$ hoch ist (Abb. 14/WII-1993-33). Bei den weiteren beiden in der Länge vollständig erhaltenen Kantenretuschen handelt es sich zum einen um eine die ganze sinistrolaterale Kante einnehmende, schräg konvexe Schuppenretusche, die eine Länge von 38,1 mm und eine Höhe von 3,2 mm aufweist (Abb. 14/WII-1993-45-1), und zum anderen um eine ventrale, gerade Schuppenretusche an der dextrolateralen Kante proximal mit einer Länge von 23,1 mm und einer Höhe von $1,9 \mathrm{~mm}$. Insgesamt wurde nur eine Klinge mit zwei modifizierten Kanten nachgewiesen; da das Werkzeug nicht vollständig erhalten ist, kann nicht ausgeschlossen werden, dass es sich eventuell um eine gebrochene Spitzklinge handelt. Des Weiteren befindet sich bei zwei Kantenretuschen die Modifikation auf der Ventralfläche. Eine Präferenz für dextro- oder sinistrolateral konnte nicht festgestellt werden. Am häufigsten wurden Schuppenretuschen an den Kanten angebracht und meistens verlaufen die Kantenretuschen 

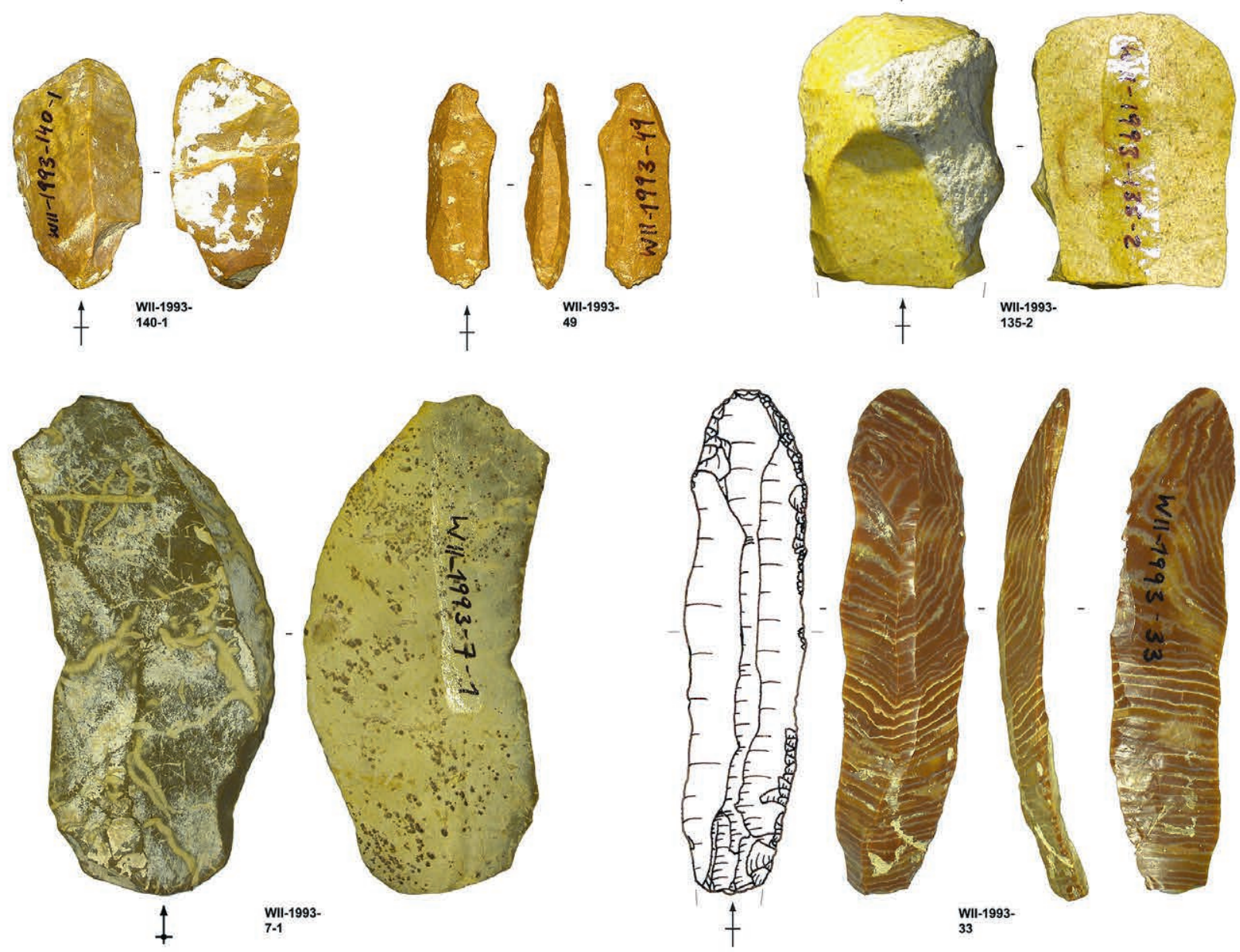

${ }_{33}^{\text {WII-1993- }}$
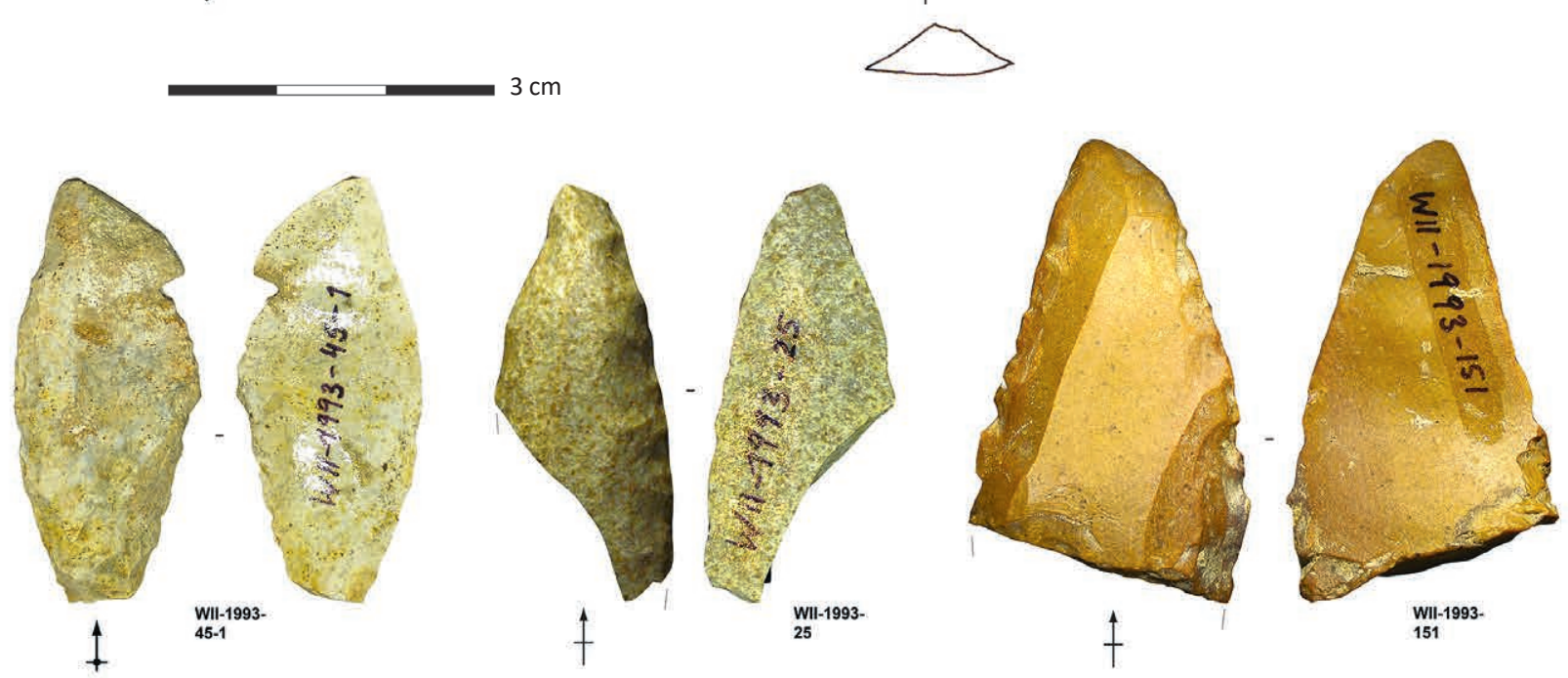

Abb. 14. Willendorf II, AH 8: Werkzeuge. - Oberste Reihe von links nach rechts: WII-1993-140-1, Ausgesplittertes Stück und Kantenretusche. - WII-1993-49, Stichel. - WII-1993-135-2, Kratzer und Kantenretusche. - Mittlere Reihe: WII-1993-7-1, Kantenretusche. - WII-1993-33, Kantenretusche (Zeichnung: F. Brodbeck). - Unterste Reihe: WII-1993-45-1, Kantenretusche. - WII-1993-25, Spitzklinge. - WII-1993-151, Spitzklinge. 


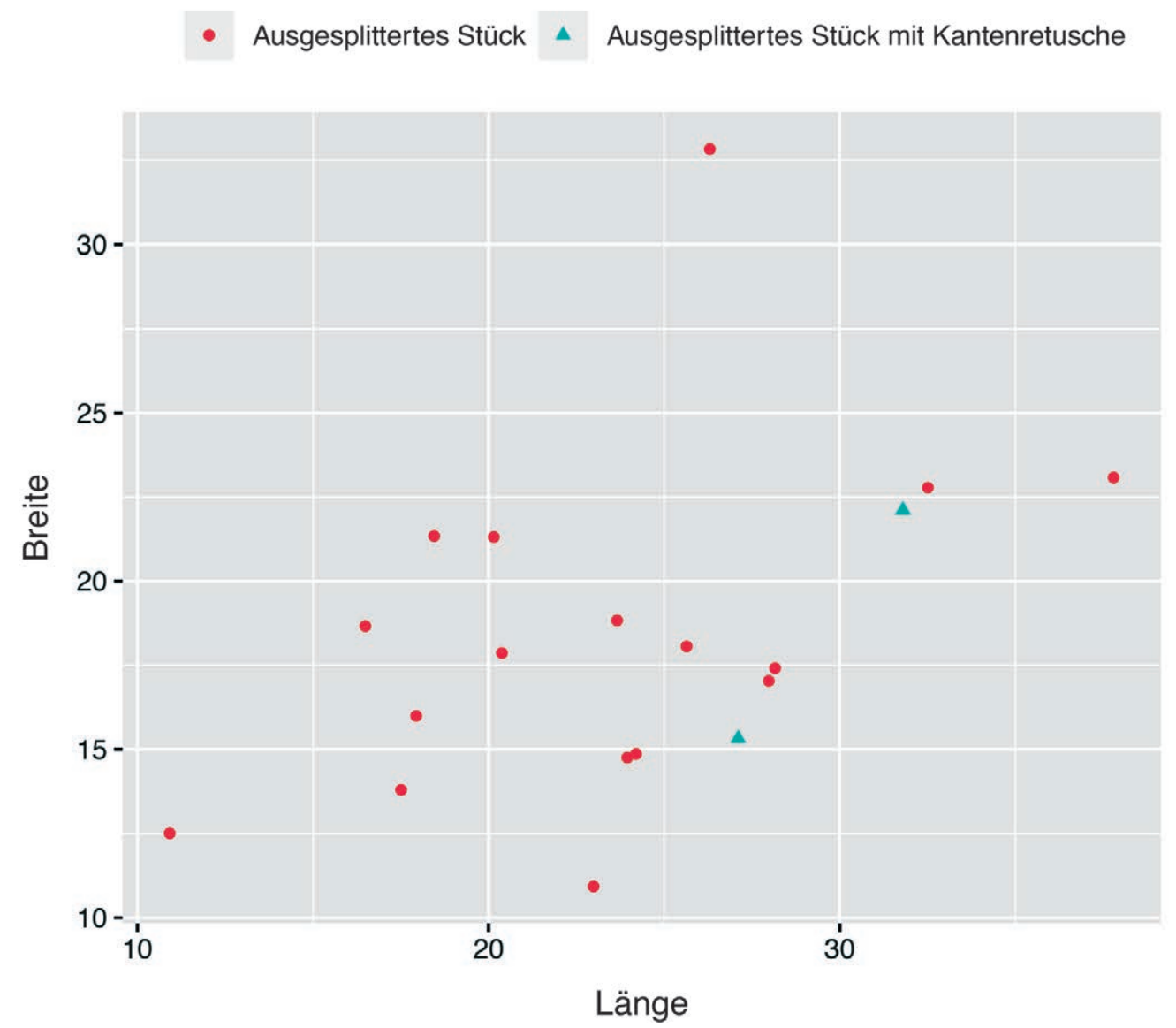

Abb. 15. Willendorf II, AH 8: Scatterplot der Länge und Breite der Ausgesplitterten Stücke und der Ausgesplitterten Stücke mit Kantenretusche. Alle Angaben in Millimeter.

gerade. Die Höhe ist bei allen Kantenretuschen vollständig erhalten und daher konnte ermittelt werden, dass die Kantenretuschen 1,2 bis maximal $7 \mathrm{~mm}$ hoch sind.

Bohrer und Kantenretusche: Der einzige Bohrer besteht aus baltischem Feuerstein und wurde an einer Klinge angebracht. Zusätzlich wurde die sinistrolaterale Kante noch mit einer schräg geraden, starken, alternierenden Retusche, die in der Länge gebrochen ist und eine Höhe von 2,1 mm aufweist, versehen.

Endretusche: Beide Endretuschen wurden dorsal am Distalende angebracht und sind in der Länge vollständig erhalten. Bei der ersten Endretusche wurde das Ende mit einer schräg konvexen, starken Retusche, die eine Länge von 11,3 $\mathrm{mm}$ und eine Höhe von $3 \mathrm{~mm}$ aufweist, versehen. Bei der zweiten handelt es sich um eine konvexe, feine Retusche mit stumpfer Kante, die 8,6 mm lang und 1,3 mm hoch ist.

Kratzer: Ein einfacher Kratzer an einem Abschlag aus Quarzit liegt vor. Am Distalende wurde eine Kratzerkappe angelegt, die aber in der Länge nicht vollständig erhalten ist und eine Höhe von 5,3 mm hat.
Kratzer und Kantenretusche: Außerdem treten zwei weitere Kratzer kombiniert mit Kantenretuschen im Inventar auf. Zum einen liegt ein einfacher Kratzer aus Kieselkalk vor, dessen Ausgangsform ein Klingenabschlag war und der distal eine Kratzerkappe mit einer Länge von 28,5 mm und einer Höhe von 9,3 mm aufweist. Hinzu tritt sinistrolateral eine schräg gerade, feine Retusche mit stumpfer Kante, die 16,2 $\mathrm{mm}$ lang und 0,9 $\mathrm{mm}$ hoch ist. Zum anderen existiert ein Klingenkratzer aus Jaspis mit einer Kratzerkappe distal, die 21,8 mm lang und 5,7 mm hoch ist (Abb. 14/WII-1993135-2). Beide Kanten wurden modifiziert, allerdings sind die Kantenretuschen nicht vollständig erhalten. Sinistrolateral wurde eine gerade Schuppenretusche und dextrolateral eine schräg konkave Stufenretusche angelegt.

Spitzklinge: Bei beiden Spitzklingen ist die Länge der Modifikation nicht vollständig erhalten. Die eine Spitzklinge besteht aus Quarzit und wurde zweikantig mit einer Schuppenretusche versehen (Abb. 14/WII-1993-151). Die Höhe der Modifikation dextrolateral mit 2,8 mm weicht von jener 


\begin{tabular}{|l|c|c|c|c|c|c|c|c|c|c|}
\hline \multirow{2}{*}{ Artefaktklasse AH 8 } & \multicolumn{2}{|c|}{ Vollständig } & \multicolumn{2}{|c|}{ Proximal } & \multicolumn{2}{c|}{ Medial } & \multicolumn{2}{c|}{ Distal } & \multicolumn{2}{c|}{ Gesamt } \\
\hline & $\mathrm{n}$ & $\%$ & $\mathrm{n}$ & $\%$ & $\mathrm{n}$ & $\%$ & $\mathrm{n}$ & $\%$ & $\mathrm{n}$ & $\%$ \\
\hline Klinge & 6 & $14 \%$ & 15 & $34,9 \%$ & 15 & $34,9 \%$ & 7 & $16,3 \%$ & 43 & $100 \%$ \\
\hline Lamelle & - & - & 2 & $16,7 \%$ & 7 & $58,3 \%$ & 3 & $25 \%$ & 12 & $100 \%$ \\
\hline Abschlag & 48 & $38,4 \%$ & 28 & $22,4 \%$ & 25 & $20 \%$ & 24 & $19,2 \%$ & 125 & $100 \%$ \\
\hline Gesamt & $\mathbf{5 4}$ & $\mathbf{3 0} \%$ & $\mathbf{4 5}$ & $\mathbf{2 5 \%}$ & $\mathbf{4 7}$ & $\mathbf{2 6 , 1} \%$ & $\mathbf{3 4}$ & $\mathbf{1 8 , 9} \%$ & 180 & $100 \%$ \\
\hline
\end{tabular}

Tab. 59. Willendorf II, AH 8: Erhaltung der Länge (in mm) bei allen Grundformen.

\begin{tabular}{|c|c|c|c|c|c|c|c|c|c|c|}
\hline \multirow[t]{2}{*}{ Artefaktklasse AH 8} & \multicolumn{2}{|c|}{$\begin{array}{c}\text { Breite } \\
\text { vollständig }\end{array}$} & \multicolumn{2}{|c|}{$\begin{array}{c}\text { Breite } \\
\text { unvollständig }\end{array}$} & \multicolumn{2}{|c|}{$\begin{array}{c}\text { Dicke } \\
\text { unvollständig }\end{array}$} & \multicolumn{2}{|c|}{$\begin{array}{c}\text { Breite und } \\
\text { Dicke unvollständig }\end{array}$} & \multicolumn{2}{|c|}{ Gesamt } \\
\hline & $\mathrm{n}$ & $\%$ & $\mathrm{n}$ & $\%$ & $\mathrm{n}$ & $\%$ & $\mathrm{n}$ & $\%$ & $\mathrm{n}$ & $\%$ \\
\hline Klinge & 38 & $88,4 \%$ & 4 & $9,3 \%$ & - & - & 1 & $2,3 \%$ & 43 & $100 \%$ \\
\hline Lamelle & 11 & $91,7 \%$ & 1 & $8,3 \%$ & - & - & - & - & 12 & $100 \%$ \\
\hline Abschlag & 68 & $54,4 \%$ & 45 & $36 \%$ & 2 & $1,6 \%$ & 10 & $8 \%$ & 125 & $100 \%$ \\
\hline Gesamt & 117 & $65 \%$ & 50 & $27,8 \%$ & 2 & $1,1 \%$ & 11 & $6,1 \%$ & 180 & $100 \%$ \\
\hline
\end{tabular}

Tab. 60. Willendorf II, AH 8: Erhaltung der Breite (in mm) und Dicke (in mm) bei allen Grundformen.

sinistrolateral mit 4,2 $\mathrm{mm}$ ab. Die andere Spitzklinge wurde aus Jaspis hergestellt (Abb. 14/WII-1993-25). Wie bei der ersten Spitzklinge wurde an beiden Kanten eine Schuppenretusche angelegt. Allerdings liegt die Höhe der Modifikation dextrolateral bei 6,1 mm und sinistrolateral bei 4,6 $\mathrm{mm}$. Gezähntes Stück: Das Gezähnte Stück aus Quarzit wurde an einem Abschlag hergestellt. Dextrolateral wurden zwei Einbuchtungen festgestellt. Allerdings ist ein Teil der dextrolateralen Kante ausgebrochen und daher ist die Modifikation in der Länge nicht komplett erhalten.

\subsubsection{Erhaltungszustand}

Die meisten Stücke sind vollständig erhalten, gefolgt von den Medialfragmenten (Tab. 59). Doch diese Gesamtverteilung täuscht darüber hinweg, dass keine einzige Lamelle vollständig erhalten blieb und lediglich $14 \%$ der Klingen. Bei den Klingen treten am häufigsten Medial- und Distalfragmente mit jeweils 34,9 \% auf. Über die Hälfte der Lamellen ist medial erhalten. Die Abschläge halten den höchsten Anteil an vollständig erhaltenen Stücken und aufgrund der im Vergleich zu Klingen und Lamellen hohen Stückzahl wird die Gesamtverteilung dementsprechend beeinflusst. Am zweithäufigsten treten mit 22,4 \% distal erhaltene Abschläge auf. Es liegt kein Indiz für einen intentionellen Charakter dieser Brüche vor.
Bei Klingen und Lamellen überwiegt deutlich der Anteil an in der Breite und Dicke vollständigen Stücken (Tab. 60). 8,3 \% der Lamellen und 9,3 \% der Klingen sind lateral gebrochen. Auch bei den Abschlägen dominieren die in der Breite und Dicke vollständigen Stücke, allerdings liegt der Anteil bei nur 54,4 \% und ist damit im Vergleich zu Klingen und Lamellen deutlich niedriger. Bei $36 \%$ der Abschläge wurde ein lateraler Bruch festgestellt. $8 \%$ der Abschläge sind in Breite und Dicke unvollständig.

Der Anteil an Stücken mit Kantenbeschädigung ist relativ hoch. Bei den wenigen Lamellen weist die Hälfte keine Kantenbeschädigungen auf. Bei Klingen und Abschlägen umfassen diejenigen Stücke ohne Beschädigung jeweils lediglich ca. $10 \%$. Kantenbeschädigungen wurden bei über $60 \%$ der Klingen und ebenso der Abschläge festgestellt. Des Weiteren wurden bei 9,3\% der Klingen und bei $12 \%$ der Abschläge Aussplitterungen aufgrund der Nutzung als Ausgesplitterte Stücke nachgewiesen.

Der Anteil an thermisch veränderten Grundformen fällt mit 6,3\% relativ gering aus. Allerdings liegen häufig Kombinationen an Merkmalen vor, die für Hitzebeschädigung typisch sind. Die meisten der Stücke mit thermischer Einwirkung dürften somit zufällig in Berührung mit Feuer gekommen sein. Mehr als die Hälfte der Trümmer stellen Hitzetrümmer dar, bei welchen eben gerade die thermische 
Einwirkung zur Unbrauchbarkeit für jeglichen Gebrauch oder Weiterverarbeitung führte.

\subsubsection{Schlagsteine}

Vier Stücke des Steinartefaktinventars wiesen typische Schlagspuren auf und dürften als Schlagsteine verwendet worden sein. Zwei dieser Schlagsteinfragmente bestehen aus einem nicht näher identifizierbaren metamorphen Gestein. Zwei weitere Fragmente sind aus Serpentinit und konnten zusammengepasst werden (Tab. 61). Auch in Felgenhauers Monografie wird beschrieben, dass Serpentinite als Schlagsteine herangezogen wurden. ${ }^{155}$ Somit ist der Einsatz des direkten, harten Schlages neben anderen Schlagtechniken in AH 8 auch durch das Vorhandensein der dafür notwendigen Schlaginstrumente belegt.

\subsubsection{Manuporte}

Insgesamt liegen 45 weitere mineralische Objekte vor, wovon 43 keine makroskopischen Bearbeitungsspuren aufweisen und zwei so stark verbrannt sind, dass die Gesteinsart nicht mehr bestimmt werden konnte. Diese beiden stark verbrannten Stücke, die zusammengepasst werden konnten, zeigen eine rote Farbveränderung und Craquelierung. Den höchsten Anteil der übrigen Manuporte macht Glimmerschiefer ( $n=18)$ aus, gefolgt von Amphibolit $(n=15)$. Je ein Stück liegt aus Quarzit und Marmor vor. Bei acht Stücken konnte nicht festgestellt werden, um welche Art metamorphes Gestein es sich handelt. Die Dimensionen und das Gewicht sind sehr unterschiedlich bei den verschiedenen Manuporten (Tab. 62). Der Block aus Marmor stellt sowohl bei Länge, Breite, Dicke als auch beim Gewicht immer den Maximalwert.

Insgesamt konnten unter den Manuporten auch $\mathrm{Zu}$ sammenpassungen gefunden werden (Tab. 61). In allen acht Fällen handelt es sich um Aneinanderpassungen, darunter sind vier Komplexe mit zwei Stücken, drei Komplexe mit drei Stücken und ein Komplex mit vier Stücken. Allerdings dürften die Brüche zu dem Zusammenpassungskomplex, der vier Stücke umfasst, aufgrund der frisch anmutenden Bruchstellen eher modern entstanden sein. Die genaue Verwendung der Manuporte bleibt unklar.

\subsection{Weitere Fundgattungen}

\subsubsection{Ocker und Rötel/Hämatit}

In beiden $\mathrm{AH}$ wurden Hämatitkonglomerate mit Bearbeitungsspuren gefunden. Es gibt verschiedene Nutzungsbereiche dieser Materialien. Erstens wurde Ocker

155 Felgenhauer 1956-1959, 106.

\begin{tabular}{|c|c|c|c|}
\hline 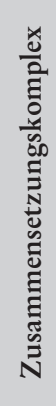 & 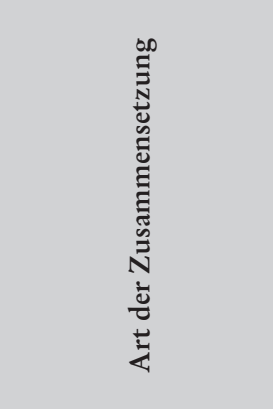 & 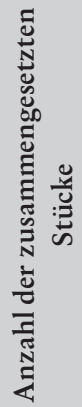 & 竭 \\
\hline 010 & $\begin{array}{c}\text { Aneinanderpassung - } \\
\text { Manuport }\end{array}$ & 2 & $\begin{array}{l}\text { WII-1993-81-4 } \\
\text { WII-1993-81-10 }\end{array}$ \\
\hline 011 & $\begin{array}{c}\text { Aneinanderpassung - } \\
\text { Manuport }\end{array}$ & 2 & $\begin{array}{l}\text { WII-1993-81-5 } \\
\text { WII-1993-81-8 }\end{array}$ \\
\hline 012 & $\begin{array}{c}\text { Aneinanderpassung - } \\
\text { Manuport }\end{array}$ & 3 & $\begin{array}{l}\text { WII-1993-44-2 } \\
\text { WII-1993-44-3 } \\
\text { WII-1993-44-4 }\end{array}$ \\
\hline 013 & $\begin{array}{c}\text { Aneinanderpassung - } \\
\text { Manuport }\end{array}$ & 3 & $\begin{array}{l}\text { WII-1993-145-1 } \\
\text { WII-1993-145-2 } \\
\text { WII-1993-145-3 }\end{array}$ \\
\hline 014 & $\begin{array}{c}\text { Aneinanderpassung - } \\
\text { Manuport }\end{array}$ & 2 & $\begin{array}{l}\text { WII-1993-99-2 } \\
\text { WII-1993-99-4 }\end{array}$ \\
\hline 016 & $\begin{array}{c}\text { Aneinanderpassung - } \\
\text { Manuport }\end{array}$ & 2 & $\begin{array}{l}\text { WII-1993-126-1 } \\
\text { WII-1993-126-3 }\end{array}$ \\
\hline 021 & $\begin{array}{c}\text { Aneinanderpassung - } \\
\text { Manuport }\end{array}$ & 3 & $\begin{array}{l}\text { WII-1993-69-1 } \\
\text { WII-1993-69-2 } \\
\text { WII-1993-69-3 }\end{array}$ \\
\hline 024 & $\begin{array}{c}\text { Aneinanderpassung - } \\
\text { Manuport }\end{array}$ & 4 & $\begin{array}{l}\text { WII-1993-87-2 } \\
\text { WII-1993-87-3 } \\
\text { WII-1993-87-4 } \\
\text { WII-1993-87-5 }\end{array}$ \\
\hline 026 & $\begin{array}{c}\text { Aneinanderpassung - } \\
\text { Schlagstein }\end{array}$ & 2 & $\begin{array}{l}\text { WII-1993-56-1 } \\
\text { WII-1993-56-2 }\end{array}$ \\
\hline
\end{tabular}

Tab. 61. Willendorf II, AH 8: Zusammensetzungskomplexe der Manuporte und Schlagsteine.

\begin{tabular}{|l|c|c|c|c|}
\hline AH 8 & Länge & Breite & Dicke & Gewicht \\
\hline Anzahl & 45 & 45 & 45 & 45 \\
\hline Min & 10,4 & 5,54 & 3,32 & 0,3 \\
\hline Max & 134,16 & 89,02 & 45,49 & 623,1 \\
\hline Mittelwert & 38,86111 & 24,68822 & 12,28667 & 35,62667 \\
\hline Stand. Abw. & 24,02763 & 16,80411 & 8,49201 & 97,01001 \\
\hline Median & 32,43 & 19,75 & 8,65 & 7,1 \\
\hline
\end{tabular}

Tab. 62. Willendorf II, AH 8: Maße (Länge, Breite und Dicke in $\mathrm{mm}$; Gewicht in g) aller Manuporte. 
als Farbstoff verwendet, um andere Objekte, Tiere, Pflanzen oder Menschen einzufärben. Als Beispiele müssen neben der Venus I von Willendorf, die rote Farbspuren aufwies, ${ }^{156}$ auch Schmuckschnecken, Dentalien, Knochen und Knochenanhänger genannt werden, wie aus anderen Gravettienfundstellen bekannt ist. ${ }^{157}$ Wie zum Beispiel die Doppelsäuglingsbestattung von Krems-Wachtberg ${ }^{158}$ oder die Dreifachbestattung von Dolní Věstonice $\mathrm{II}^{159}$ zeigen, treten Mengen an Farbstoff auch in Gräbern immer wieder auf. ${ }^{160}$ Natürlich ist nicht auszuschließen, dass auch die Menschen ihre Körper durch Bemalung mit rotem Farbstoff dekorierten. ${ }^{161}$ Des Weiteren ergaben Residuenuntersuchungen an Steinwerkzeugen aus Rose Cottage Cave und Sibudu Cave (Südafrika), dass diese geschäftet waren und roter Ocker auch als ein Bestandteil des Klebemittels verwendet wurde. ${ }^{162}$ Außerdem könnte Ocker auch als Hautschutz vor Mücken und Kälte Verwendung gefunden haben. ${ }^{163}$ Wozu nun genau die Ockerstücke dieser beiden Inventare gedient haben, konnte nicht geklärt werden.

Von den insgesamt vier bearbeiteten Hämatitkonglomeraten aus AH 6 weisen zwei Stücke Farbvariationen auf, die auf Hitzeeinwirkung hindeuten könnten. Die Ausmaße und vor allem das Gewicht sind sehr unterschiedlich bei den Ockerstücken (Tab. 63). Vor allem ein Hämatitkonglomerat sticht größen- und gewichtsmäßig hervor. Auch in dem Inventar von AH 8 wurden vier bearbeitete Hämatitkonglomerate nachgewiesen. Im Gegensatz zu den Ockerstücken aus AH 6 sind die Objekte von ihren Ausmaßen her eher in einem Bereich, beim Gewicht sind die Abweichungen nicht annähernd so groß (Tab. 64).

\subsubsection{Knochen}

Von dem 19 Stücke umfassenden Knochenmaterial stammen zwei aus AH 6 und 16 aus AH 8. Ein Knochen wurde in der modernen Humusschicht aufgefunden und daher bei der Auswertung nicht berücksichtigt. Wie für die Faunenreste, die bei der Grabung 2006-2011 aus den AH 6 und 8 geborgen wurden, ${ }^{164}$ zeigen auch die Knochen der Grabung 1993 aus beiden AH eine sehr schlechte Erhaltung

156 Angeli 1989, 45. - ANTL-Weiser 2008a. - ANTL-Weiser 2008b. 157 Simon 1996, 204. - ANTL-Weiser et al. 2010.

158 HäNDEL et al. 2009, 191.

159 Klíma 1995.

160 Petтitt 2011. - Riel-Salvatore, Gravel-Miguel 2013. SIKORA et al. 2017, Supporting Information 7-8.

161 Henshilwood et al. 2011, 219.

162 WADLEY 2005.

163 Henshilwood et al. 2011, 219.

164 Nigst et al. 2008b, 14

\begin{tabular}{|l|c|c|c|c|}
\hline AH 6 & Länge & Breite & Dicke & Gewicht \\
\hline Anzahl & 4 & 4 & 4 & 4 \\
\hline Min & 6,41 & 5,05 & 1,52 & 0,1 \\
\hline Max & 62,38 & 38,6 & 12,09 & 39,5 \\
\hline Mittelwert & 38,86111 & 24,68822 & 12,28667 & 35,62667 \\
\hline Stand. Abw. & 26,61915 & 16,02757 & 5,152594 & 18,7857 \\
\hline Median & 21,085 & 14,535 & 5,91 & 3,85 \\
\hline
\end{tabular}

Tab. 63. Willendorf II, AH 6: Maße (Länge, Breite und Dicke in mm; Gewicht in g) aller Hämatitkonglomerate.

\begin{tabular}{|l|c|c|c|c|}
\hline AH 8 & Länge & Breite & Dicke & Gewicht \\
\hline Anzahl & 4 & 4 & 4 & 4 \\
\hline Min & 12,94 & 7,8 & 4,67 & 0,5 \\
\hline Max & 29,52 & 17,21 & 14,52 & 7 \\
\hline Mittelwert & 22,0875 & 14,085 & 10,595 & 4,4 \\
\hline Stand. Abw. & 7,537035 & 4,415643 & 4,251372 & 2,824889 \\
\hline Median & 22,945 & 15,665 & 11,595 & 5,05 \\
\hline
\end{tabular}

Tab. 64. Willendorf II, AH 8: Maße (Länge, Breite und Dicke in $\mathrm{mm}$; Gewicht in g) aller Hämatitkonglomerate.

aufgrund der Verwitterung der Knochenoberfläche, der starken Versinterung und dem hohen Fragmentierungsgrad in der Form von alten Trockenrissen. Diese Faktoren schränken die Identifizierung von anthropogenen Modifikationen sehr ein und daher verwundert es kaum, dass keine Schnittspuren oder Auftreffpunkte von Schlagsteinen zur Knochenmarkgewinnung nachgewiesen werden konnten.

Die zwei Knochen aus AH 6 waren so stark versintert, dass keine Aussage darüber getroffen werden konnte, um welche Tiergattung es sich handelt. Einzig steht fest, dass die Knochen nicht gebrannt sind.

Die Knochen aus AH 8 lieferten mehr Informationen. Bei fünf Knochen war die Erhaltung allerdings ebenfalls so schlecht, dass auch hier lediglich festgehalten werden konnte, dass sie nicht gebrannt sind. Eines von drei als Kompakta identifizierten Stücken ist kalziniert und belegt damit anthropogenen Einfluss. Drei weitere faunistische Überreste, von denen zwei zusammengepasst werden konnten, stammen von größeren Säugetieren, d. h. von Pferd- bis Mammutgröße. Bei zwei Bruchstücken konnte klar bestimmt werden, dass es sich um Langknochen handelt. Der eine Langknochen stammt mit hoher Wahrscheinlichkeit von einem Pferd und bei dem anderen konnte lediglich festgestellt werden, dass er von einem Säugetier in Pferd-/Bisongröße 
stammen muss. Des Weiteren liegt ein Rippenfragment eines größeren Säugetiers vor. Zwei Fragmente stellen Spongiosa dar. Bei einem Fragment der beiden könnte es sich um einen Teil eines Wirbelkörpers handeln, wobei die Tiergattung nicht identifiziert werden konnte. Beim anderen konnte festgestellt werden, dass er von einem Tier in der Größenordnung zwischen Pferd und Mammut stammt. Der größte Teil der Knochen ist zwar unbestimmbar, doch bei den restlichen Knochen konnte zumindest festgestellt werden, dass es sich um Tiere in Pferd- bis Mammutgröße handeln muss. Damit werden die Ergebnisse, die die Analyse der Fauna aus der Grabung 2006 erbrachte, ${ }^{165}$ und auch Felgenhauers Angaben zur in Willendorf II vertretenen Fauna ${ }^{166}$ bestätigt und ergänzt.

\section{Diskussion und Schlusswort}

\subsection{Beschreibung der Technoökonomie und Technologie von AH 6}

Die am häufigsten verwendeten Gesteine stellen einerseits der größtenteils wohl aus den lokalen Donauschottern stammende alpine Hornstein und andererseits der nicht-lokale, importierte baltische Feuerstein dar. Da der Hauptanteil der Grundformen keine dorsalen Reste natürlicher Oberflächen aufweist und primäre Produkte gänzlich fehlen, dürften die Rohmaterialien sehr wahrscheinlich bereits vorpräpariert zur Fundstelle transportiert worden sein.

Die Abbaustrategie war auf die Produktion von eher kleinen, schmalen und länglichen Elementen, Lamellen, ausgerichtet. Für Lamellen wurden ausschließlich die am häufigsten vorkommenden Rohmaterialien, Hornstein und Feuerstein, verwendet. Dieser im Gravettien weitverbreitete Trend der Selektion dieser hochwertigen Rohmaterialien für die serielle Herstellung von Lamellen dürfte damit zusammenhängen, dass diese Grundformen für die Weiterverarbeitung zu Rückenelementen bestimmt waren und daher die genutzten Rohmaterialien die hohen technischen Ansprüche dieser speziellen Werkzeugherstellung zu Projektilen erfüllen mussten. ${ }^{167}$

Trotz des Fehlens von Kernen zeichnen sich Charakteristika der Kernabbaumethode ab. Am Beginn wurden Kernkanten angelegt, um so einen artifiziellen Leitgrat zur Eröffnung des Kernabbaus zu schaffen. Die starke Präsenz von natürlichen Oberflächen bei den Abschlägen deutet darauf hin, dass gerade diese bei der Kernpräparation am

165 NigST et al. $2008 \mathrm{~b}, 14$.

166 Felgenhauer 1956-1959, 81-83.

167 Siehe Morala, Turq 1991. - O’Farrell 2000. - Floss,

Kieselbach 2004. - Moreau 2009. - Moreau 2012.
Beginn des Abbaus anfielen. Die Reste von natürlichen Oberflächen der Grundformen insgesamt befinden sich fast ausschließlich distal oder dextrolateral. Dieses Muster dürfte widerspiegeln, dass in den anfänglichen Phasen des Kernabbaus eine Erweiterung der Abbaufläche auf die rechte Flanke stattfand, wobei der Grat zwischen den in die Flanken übergehenden und den frontalen Negativen den Leitgrat für die Gewinnung der Zielprodukte darstellte. ${ }^{168}$ Zusätzlich zeigen die distal mit natürlichen Oberflächen bedeckten länglichen Grundformen ohne Kernfuß, dass, weil eben der Abbau noch nicht so weit fortgeschritten war, die nutzbare Länge der Abbaufläche gerade ausgebaut wurde. ${ }^{169}$

Natürliche sowie glatte Flächen wurden als Schlagflächen genutzt. Als Schlagtechnik zur gezielten Lamellenproduktion bedienten sich die SteinschlägerInnen entweder des direkten, weichen, mineralischen oder des direkten, organischen Schlages. Die Zielprodukte bestätigen dies durch die dünnen sowie schmalen Schlagflächenreste, das häufige Auftreten von Lippen, die Abbauwinkel von ca. $80^{\circ}$ und die bei allen nachgewiesene dorsale Reduktion, die auf eine kontinuierliche Begradigung und Stabilisierung der Schlagflächenkante hinweist. Während der Präparation der Kerne dürfte jedoch auch der direkte, harte Schlag zum Einsatz gekommen sein.

Die vorwiegend gleichgerichteten, dorsalen Negative der gesamten Grundformen bezeugen die unidirektionale Abbaustrategie. Die Stücke mit gleichgerichteten und quer verlaufenden Negativen stehen zum einen in Zusammenhang mit der Kernkantenpräparation und zum anderen mit der Zurichtung der Lateralflächen der Kerne. Die wenigen auftretenden bidirektionalen Stücke und das einzige gegenläufige Stück dürften nicht auf eine weitere bidirektionale Abbaustrategie hindeuten, sondern auf das Anlegen einer zweiten Schlagfläche zur Wiederinstandsetzung der Abbauflächenkonvexität. ${ }^{170}$

Das Inventar umfasst Lamellen mit Kernfuß. Diese Lamellen wurden über die ganze Abbauflächehinweg abgebaut und entfernten einen Teil des der Schlagfläche gegenüberliegenden Endes des Kerns. Dieser Teil wurde entweder von natürlichen Oberflächen oder von Negativen einer zweiten gegenläufigen Schlagfläche gebildet. Sie stammen aus einer späteren Kernabbauphase, in welcher die nutzbare Länge der Abbaufläche bereits voll erschöpft war und abnahm. ${ }^{171}$

Neben der unidirektionalen, volumetrischen Lamellenabbaustrategie wurden auch Stichel als Lamellenkerne

168 Moreau 2009, 100-101.

169 Soriano, Villa, Wadley 2007, 687-688.

170 Vgl. Moreau 2009, 103-105.

171 Vgl. Soriano, Villa, Wadley 2007, 687-688. 
genutzt, wie aus anderen gravettienzeitlichen Kontexten bereits bekannt ist. ${ }^{172}$ Zwar wurde kein als Kern genutzter Sticheltyp aufgefunden, jedoch tritt eine Stichellamelle auf, welche als Beleg für diese Art der Lamellenproduktion gelten kann.

Das Werkzeugspektrum enthält zwei Rückenelemente aus alpinem Hornstein und baltischem Feuerstein. Diese bestätigen die Hypothese, dass die Lamellen an hochwertigen Rohmaterialien für die Weiterverarbeitung zu technisch aufwendigen rückengestumpften Projektilen dienten. Außerdem kommen zwei Stichel vor. Zuletzt wurden auch Kratzer und Kantenretuschen festgestellt.

Anhand der Zusammenpassungen konnte die stratigrafische Integrität belegt werden und eindeutig erwiesen werden, dass Grundformenproduktion vor Ort erfolgte.

\subsection{Beschreibung der Technoökonomie und Technologie von AH 8}

Am häufigsten wurden verschiedene lokale Varietäten von Quarzit als Ausgangsrohmaterial für die Grundformenproduktion und Werkzeugherstellung genutzt, gefolgt von ebenfalls lokalem Kieselkalk und Jaspis aus ca. 15 bis $20 \mathrm{~km}$ Entfernung. ${ }^{173}$ Die Anteile an natürlichen Oberflächen insgesamt und vor allem das Vorkommen von primären Grundformen bei diesen Rohmaterialien weist darauf hin, dass diese zumindest teilweise ohne Entrindung zur Fundstelle transportiert und erst vor Ort verarbeitet wurden. Der geringfügig vorhandene, nicht-lokale Feuerstein hingegen dürfte in bereits vorpräparierter Form zur Fundstelle gelangt sein.

Der Abbau zielte auf größere und längliche Elemente, Klingen, ab. Lamellen kommen vor, dürften aber eher das Kontinuum der Klingenherstellung in fortgeschrittenem Abbaustadium darstellen als eine unabhängige Kernabbaustrategie. Das Auftreten von Kernkantengrundformen belegt, dass die Kerne anfänglich mit einem künstlich angelegten Leitgrat präpariert wurden, um das kontrollierte Lösen einer Grundform zu ermöglichen. Die Mehrheit der länglichen Grundformen zeigt dorsal keine Reste von natürlichen Oberflächen, während bei den Abschlägen vermehrt Stücke mit Bedeckung vorhanden sind. Daher dürften zu Beginn des Kernabbaus Primärabschläge abgetrennt worden und erst im weiteren Verlauf des Abbaus nach und nach die Gewinnung von Klingen erfolgt sein.

Bei den Kernen liegen exklusiv glatte und mit natürlichen Oberflächen bedeckte Schlagflächen vor und auch die

172 Siehe Hahn 1969. - Bordes, Lenoble 2002. - Klaric, Aubry, Walter 2002. - Lucas 2002. - Le Brun-Ricalens, Brou 2003. Moreau 2009.

173 Vgl. Moreau, BrandL, Nigst 2016, 88. meisten Grundformen weisen einen glatten oder mit natürlichen Oberflächen bedeckten Schlagflächenrest auf. Kernscheiben belegen, dass während des Fortschreitens des Abbaus, wenn notwendig, eine Korrektur des Abbauwinkels vorgenommen wurde.

Die Schlagmerkmale erlaubten eine Eingrenzung der Schlagtechnik bei den Zielprodukten auf entweder den direkten, weichen, mineralischen oder den direkten, organischen Schlag. Doch weist ein relativ hoher Anteil mit im Vergleich breiteren Schlagflächenresten der Klingen keine dorsale Reduktion auf. Dies dürfte daran liegen, dass ohne diese Präparation sowohl beim direkten, weichen, mineralischen als auch beim direkten, organischen Schlag der Schlagpunkt weiter nach innen versetzt werden musste, um Schlagunfälle zu vermeiden. Die Kerne selbst weisen mehrheitlich eine derartig präparierte Schlagkante auf. Der direkte, harte Schlag dürfte bei der Kernpräparation zum Einsatz gekommen sein, wofür Abschläge mit massiven Schlagflächenresten und ohne dorsale Reduktion sprechen.

Im Gegensatz zu den Abschlägen weisen die Zielprodukte einen höheren Anteil an Federbrüchen und weniger Schlagunfälle auf, weshalb davon ausgegangen wird, dass bei der Herstellung dieser länglichen Grundformen auf eine optimale Zurichtung der Kerne geachtet wurde. Die Häufung an Schlagunfällen bei den Abschlägen dürfte nicht nur mit schlagtechnischen Faktoren, sondern auch mit der Qualität des Rohmaterials zusammenhängen.

Sowohl die Kerne als auch die dorsalen Negative bei den Grundformen belegen die vorrangig unidirektionale Abbaustrategie. Der Anteil an Grundformen mit gleichgerichteten und quer verlaufenden Negativen weist einerseits auf das Anlegen von Kernkanten und andererseits auf die Präparation der Lateralflächen der Kerne hin. Das Fehlen von präparierten Lateralflächen bei den vorliegenden Kernen deutet allerdings darauf hin, dass die meisten bis zu ihrer endgültigen Verwerfung sehr stark reduziert wurden und damit eine eventuell zu Beginn des Abbaus angebrachte Präparation an den Lateralflächen der Kerne nicht erhalten blieb. Außerdem kommen zum einen Grundformen mit bidirektionalen oder gegenläufigen Negativen und zum anderen Kerne mit Abbauflächen, die Negative von zwei gegenüberliegenden Schlagflächen aufweisen, vor. Da die Schlagflächen versetzt zueinander angelegt wurden und kein alternierender Abbau stattfand, erfolgte wahrscheinlich eher kein bidirektionaler Abbau, sondern die Wiederherstellung der Abbauflächenkonvexität.

Die letzten Negative bei den meisten Kernen sind relativ kurze Abschlagnegative, die häufig Schlagunfälle wie Angelbrüche darstellen, womit eine Verwerfung des Kerns unumgänglich war. Es zeigt sich, dass Kerne mit nur einer 
Schlag- und Abbaufläche hauptsächlich wegen ihrer Dimension und Kerne mit mehr als einer Schlagfläche wegen Angelbrüchen verworfen wurden. Dies deutet darauf hin, dass die unidirektionale Abbaustrategie von einer einzigen Schlagfläche auf eine Abbaufläche von den Steinschlägern/ Steinschlägerinnen angestrebt wurde und nur gegebenenfalls, wenn Schlagunfälle die Unbrauchbarkeit der Abbaufläche bewirkten, weitere Schlag- bzw. Abbauflächen angelegt wurden.

Der Werkzeugbestand umfasst verschiedene Typen. Der dominierende Werkzeugtyp sind Ausgesplitterte Stücke, wobei hauptsächlich Abschläge als Ausgangsformen herangezogen wurden. Zusammenpassungen von Ausgesplitterten Stücken mit ihren Abfällen belegen die unmittelbare Verwendung an der Fundstelle. Spitzklingen treten im Werkzeugspektrum auf und zusätzlich wurden auch Stichel, ein Bohrer mit Kantenretusche und ein gezähntes Stück festgestellt. Zuletzt kommen noch Kratzer, Kantenretuschen und Endretuschen vor.

Die Zusammenpassungen bestätigen einerseits, dass die Artefakte aus einem sicheren, stratigrafischen Kontext stammen und andererseits, dass die Grundformenproduktion vor Ort stattfand.

\subsection{Vergleich zwischen den lithischen Inventaren von AH 6 und $\mathrm{AH} 8$}

Da es durchaus Kontroversen gibt, ob diese beiden Schichten in der Gliederung des Gravettien zu derselben Phase ${ }^{174}$ oder in ein älteres und jüngeres Stadium gehören, ${ }^{175}$ wird im Folgenden ein Vergleich zwischen AH 6 und AH 8 vorgenommen.

\subsubsection{Rohmaterial}

AH 6 zeichnet sich im Gegensatz zu AH 8 durch einen höheren Anteil an nicht-lokalem Rohmaterial aus. Das Inventar von AH 6 umfasst 33,3 \% nicht-lokales Rohmaterial, während in AH 8 Rohmaterialien, die von einer Distanz weiter als $10 \mathrm{~km}$ transportiert wurden, nur $24 \%$ ausmachen. Baltischer Feuerstein, der aufgrund seiner Homogenität von höherer Qualität ist und einen kontrollierteren sowie effizienteren Abbau zulässt, ${ }^{176}$ macht in AH 6 27,5 \% aus. Insgesamt dominieren in diesem $\mathrm{AH}$ die hochwertigen Rohmaterialien Hornstein und baltischer Feuerstein mit 86,3 \%, und auch der Großteil der Werkzeuge sowie spezielle Werkzeugtypen, wie Rückenelemente, wurden aus diesen Rohmaterialien gefertigt. In AH 8 hingegen wurden bevorzugt die lokalen Quarzite mit 41,2 \% und Kieselkalke mit $24 \%$ sowie Jaspise aus ca. 15 bis $20 \mathrm{~km}$ Distanz mit $16,7 \%$ verarbeitet. Interessanterweise wurde Jaspis am häufigsten für die Werkzeugherstellung herangezogen, gefolgt von Quarzit. Feuerstein kommt mit nur 3,4 \% in diesem Horizont geringfügig vor. Auffällig ist, dass kein einziger alpiner Hornstein in AH 8 nachgewiesen wurde. In beiden Schichten zeichnete sich ein gleich hoher Anteil an Radiolariten ab. Generell ist das Rohmaterialspektrum des AH 8 breiter gefächert als das von AH 6. Anhand der dorsalen Anteile von natürlichen Oberflächen bei den Rohmaterialien zeigt sich, dass in AH 6 die primären Elemente der Abbaukette bei allen Rohmaterialien zur Gänze fehlen, während hingegen in AH 8 diese Produkte aus der anfänglichen Phase des Abbaus sehr wohl vor allem bei den drei dominierenden Rohmaterialien festgestellt wurden. In beiden Schichten treten ausschließlich Grundformen aus baltischem Feuerstein auf, die entweder keine oder eine geringe (d. h. 1-33\%) dorsale Bedeckung mit natürlichen Oberflächen aufweisen.

\subsubsection{Abbaustrategien}

Beide Inventare zeigen die tendenzielle Bestrebung nach länglichen Zielprodukten an, was auch anhand des Längen-Breiten-Verhältnisses, des sogenannten Laminaritätswertes, ${ }^{177}$ ermittelt wurde (Tab. 65). Zwar ist der Unterschied nicht signifikant (Wilcoxon-Mann-Whitney-Test, $\mathrm{W}=433, \mathrm{p}=0,4082$ ) und auch die Anzahl der in der Länge und Breite vollständig erhaltenen Grundformen sehr gering, dennoch wird die Dominanz von länglichen Grundformen in AH 6 deutlicher als in $\mathrm{AH} 8$.

\begin{tabular}{|l|c|c|}
\hline & AH 6 & AH 8 \\
\hline Anzahl & 26 & 38 \\
\hline Mittelwert & 1,82 & 1,51 \\
\hline Stand. Abw. & 0,43 & 0,26 \\
\hline Varianz & 1,11 & 0,62 \\
\hline F= & \multicolumn{2}{|c|}{1,7769} \\
\hline W= & \multicolumn{2}{|c|}{433} \\
\hline$p=$ & 0,4082 \\
\hline
\end{tabular}

Tab. 65. Willendorf II, AH 6 und 8: Vergleich der Laminaritätswerte der in Länge und Breite vollständig erhaltenen Grundformen (Wilcoxon-Mann-Whitney-Test). Maßangaben in Millimeter.

177 Siehe Adler 2002, 196. - Tostevin 2003b, 85.

174 Siehe Svoboda, LožEK, VLČEK 1996, 138.

175 Siehe Oтте 1991, 48.

176 Přichystal 2013. 
Der Abbau in AH 6 war nicht nur auf die Produktion von länglichen Elementen, sondern im Speziellen auf Lamellen ausgerichtet, während sich in AH 8 die Klingenherstellung als vorherrschend herausstellte (Abb. 16). Sowohl in der Länge, Breite als auch Dicke sind die Grundformen aus AH 6 signifikant kleiner (Wilcoxon-Mann-Whitney-Test, $\mathrm{W}=3319$, p <0,0001), schmaler (Wilcoxon-Mann-Whitney-Test, W=2841,5, p <0,0001) und dünner (Wilcoxon-Mann-Whitney-Test, W=1926, p <0,0001) als in AH 8 (Tab. 66-68).

Beim Ablauf des Abbaus zeigen sich gewisse Parallelen zwischen AH 6 und AH 8. In beiden Schichten wurde der Abbau mit der Einrichtung von Kernkanten eingeleitet und Primärabschläge wurden bei der anfänglichen Kernpräparation gelöst. Die SteinschlägerInnen aus beiden Horizonten nutzen hauptsächlich natürliche sowie glatte Flächen als Schlagflächen belegt durch Grundformen und Kerne. Kernscheiben wurden ausschließlich in AH 8 dokumentiert, die diese Form der Korrektur des Abbauwinkels während des Abbaus belegen.

In AH 6 zeigte sich bei den Grundformen mit dorsaler Bedeckung mit natürlichen Oberflächen ein vermehrtes Auftreten von solchen mit distalen oder dextrolateralen Resten, welches auf eine bestimmte Vorgehensweise bei der Erweiterung der Abbaufläche hindeutet. Ein derartiges Muster konnte in AH 8 nicht festgestellt werden.

In beiden Inventaren wurde für die Herstellung der Zielprodukte entweder der direkte, weiche, mineralische oder der direkte, organische Schlag verwendet, während bei der Präparation der Kerne auch der direkte, harte Schlag zum Einsatz kam. Die Zielprodukte beider Horizonte bestätigen dies durch das häufige Vorkommen von Lippen und den Abbauwinkeln um $80^{\circ}$. Die Schlagflächenreste der Klingen und Lamellen in beiden Inventaren fallen schmal und dünn aus, vor allem verglichen mit den jeweiligen Abschlägen. Allerdings zeigt sich, dass die Schlagflächenreste der Grundformen in AH 6 signifikant schmäler (Wilcoxon-Mann-Whitney-Test, W=715, p <0,0001) und dünner (Wilcoxon-Mann-Whitney-Test, W=540, p <0,0001) sind als in AH 8 (Tab. 69, 70) und ebenso verhält es sich bei den länglichen Zielprodukten (Tab. 71, 72). Da die Schlagflächenrest-Dicke eine Variable darstellt, die Einfluss auf die Länge und Dicke der Grundformen hat ${ }^{178}$ und ein bei beiden Inventaren relativ konstantes Intervall des Abbauwinkels besteht, der ebenso entscheidend für die Länge und Dicke ist, liegt eine Übereinstimmung mit den Resultaten zu Längen und Dicken vor. Die Zielprodukte aus AH 8 mit den breiteren und dickeren Schlagflächenresten sind eben

\begin{tabular}{|l|c|c|}
\hline & AH 6 & AH 8 \\
\hline Anzahl & 31 & 54 \\
\hline Mittelwert & 18,77 & 37,34 \\
\hline Stand. Abw. & 2,66 & 5,34 \\
\hline Varianz & 52,59 & 382,53 \\
\hline F= & \multicolumn{2}{|c|}{7,2746} \\
\hline W= & \multicolumn{2}{|c|}{3319} \\
\hline$p=$ & $<0,0001$ \\
\hline
\end{tabular}

Tab. 66. Willendorf II, AH 6 und 8: Vergleich der Länge (in mm) zwischen allen in der Länge vollständig erhaltenen Grundformen (Wilcoxon-Mann-Whitney-Test).

\begin{tabular}{|l|c|c|}
\hline & AH 6 & AH 8 \\
\hline Anzahl & 62 & 99 \\
\hline Mittelwert & 12,15 & 24,08 \\
\hline Stand. Abw. & 1,78 & 3,32 \\
\hline Varianz & 49,13 & 277,55 \\
\hline F= & \multicolumn{2}{|c|}{5,6493} \\
\hline W= & \multicolumn{2}{|c|}{2841,5} \\
\hline$p=$ & $<0,0001$ \\
\hline
\end{tabular}

Tab. 67. Willendorf II, AH 6 und 8: Vergleich der Breite (in mm) zwischen allen in der Breite vollständig erhaltenen Grundformen (Wilcoxon-Mann-Whitney-Test).

\begin{tabular}{|l|c|c|}
\hline & AH 6 & AH 8 \\
\hline Anzahl & 85 & 147 \\
\hline Mittelwert & 3,35 & 7,38 \\
\hline Stand. Abw. & 0,56 & 0,81 \\
\hline Varianz & 6,19 & 27,36 \\
\hline F= & \multicolumn{2}{|c|}{3,6452} \\
\hline W= & \multicolumn{2}{|c|}{1926} \\
\hline$p=$ & $<0,0001$ \\
\hline
\end{tabular}

Tab. 68. Willendorf II, AH 6 und 8: Vergleich der Dicke (in mm) zwischen allen in der Dicke vollständig erhaltenen Grundformen (Wilcoxon-Mann-Whitney-Test).

auch länger und dicker als jene aus $\mathrm{AH}$ 6. Außerdem zeigen alle Klingen und Lamellen aus AH 6 eine dorsale Reduktion, wohingegen in AH 8 ein hoher Anteil der Klingen diese Kantenpräparation an den Schlagflächen der Kerne 


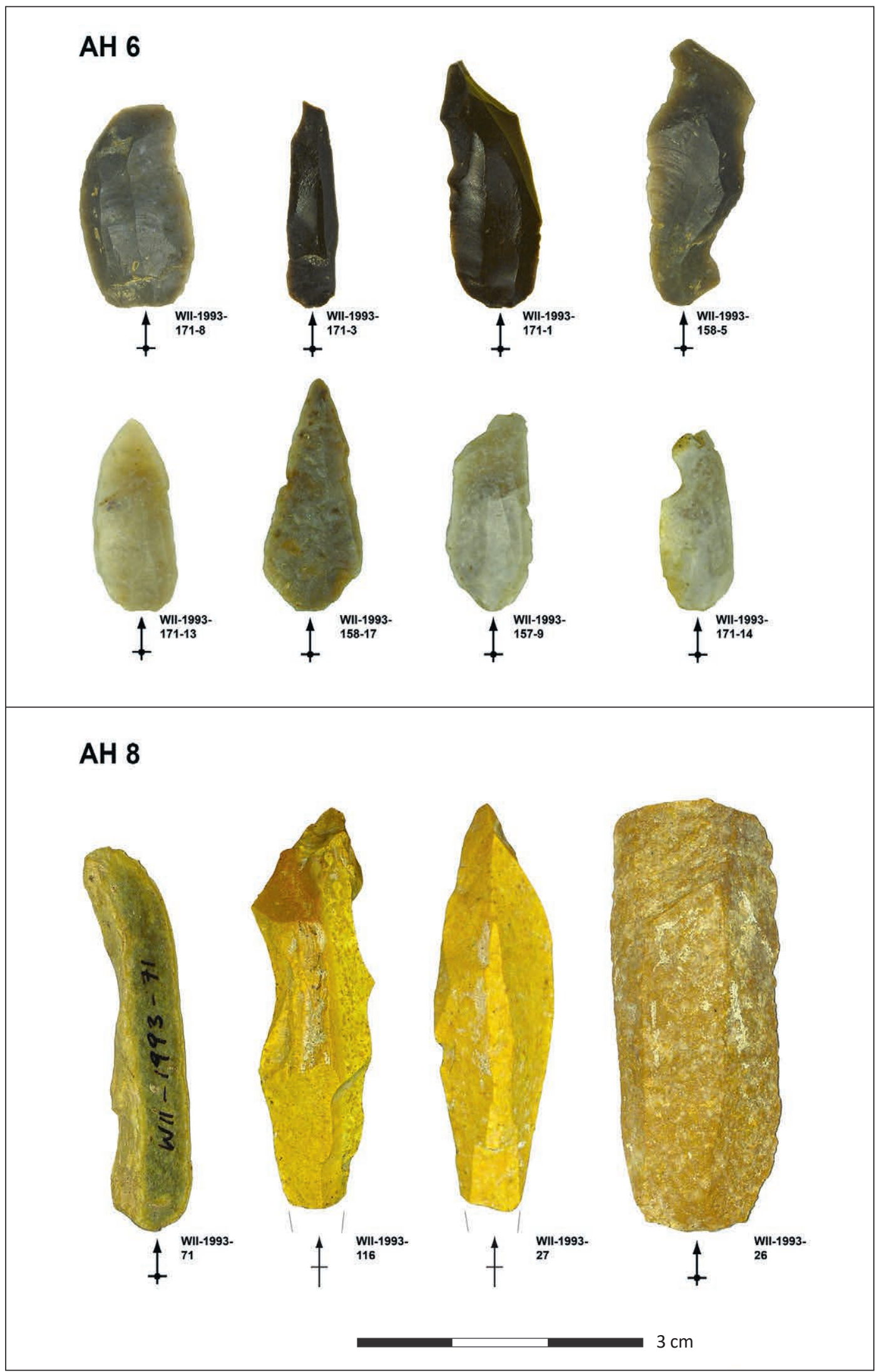

Abb. 16. Willendorf II: Zielprodukte aus AH 6 und AH 8. 


\begin{tabular}{|l|c|c|}
\hline & AH 6 & AH 8 \\
\hline Anzahl & 44 & 82 \\
\hline Mittelwert & 5,55 & 13,85 \\
\hline Stand. Abw. & 1,37 & 2,02 \\
\hline Varianz & 20,4 & 84,53 \\
\hline F= & \multicolumn{2}{|c|}{4,1436} \\
\hline W= & \multicolumn{2}{|c|}{715} \\
\hline$p=$ & $<0,0001$ \\
\hline
\end{tabular}

Tab. 69. Willendorf II, AH 6 und 8: Vergleich der SchlagflächenrestBreiten (in $\mathrm{mm}$ ) zwischen allen vollständig und proximal erhaltenen Grundformen (Wilcoxon-Mann-Whitney-Test).

\begin{tabular}{|l|c|c|}
\hline & AH 6 & AH 8 \\
\hline Anzahl & 21 & 22 \\
\hline Mittelwert & 2,77 & 7,34 \\
\hline Stand. Abw. & 0,73 & 1,71 \\
\hline Varianz & 2,56 & 14,92 \\
\hline F= & \multicolumn{2}{|c|}{$\mathbf{5 , 8 2 6 6}$} \\
\hline W= & \multicolumn{2}{|c|}{$<0,5$} \\
\hline$p=$ & \multicolumn{2}{|c|}{$<0001$} \\
\hline
\end{tabular}

Tab. 71. Willendorf II, AH 6 und 8: Vergleich der SchlagflächenrestBreiten (in $\mathrm{mm}$ ) zwischen allen vollständig und proximal erhaltenen Klingen und Lamellen (Wilcoxon-Mann-Whitney-Test).

nicht aufweist. Dies dürfte damit zusammenhängen, dass ohne diese Präparation sowohl beim direkten, weichen, mineralischen als auch beim direkten, organischen Schlag der Schlagpunkt weiter nach innen versetzt werden musste, um Schlagunfälle zu vermeiden.

Der Abbau war in beiden Schichten unidirektional ausgerichtet. Die Stücke mit gleichgerichteten und quer verlaufenden Negativen stehen zum einen in Zusammenhang mit der Kernkantenpräparation und zum anderen mit der Zurichtung der Lateralflächen der Kerne. Die bidirektionalen und gegenläufigen Grundformen sowie die Kerne in AH 8 belegen, dass weitere Schlagflächen gegenüber der ursprünglichen Hauptschlagfläche angelegt wurden, um die Abbauflächenkonvexität aufrecht zu erhalten oder wiederherzustellen. ${ }^{179}$

In AH 6 wurde neben der unidirektionalen, volumetrischen Lamellenabbaustrategie auch die Nutzung von

179 Siehe Moreau 2009, 103-105.

\begin{tabular}{|l|c|c|}
\hline & AH 6 & AH 8 \\
\hline Anzahl & 44 & 82 \\
\hline Mittelwert & 1,41 & 5,38 \\
\hline Stand. Abw. & 0,4 & 1,28 \\
\hline Varianz & 1,73 & 34,11 \\
\hline F= & \multicolumn{2}{|c|}{19,698} \\
\hline W= & \multicolumn{2}{|c|}{540} \\
\hline$p=$ & $<0,0001$ \\
\hline
\end{tabular}

Tab. 70. Willendorf II, AH 6 und 8: Vergleich der SchlagflächenrestDicken (in $\mathrm{mm}$ ) zwischen allen vollständig und proximal erhaltenen Grundformen (Wilcoxon-Mann-Whitney-Test).

\begin{tabular}{|l|c|c|}
\hline & AH 6 & AH 8 \\
\hline Anzahl & 21 & 22 \\
\hline Mittelwert & 0,65 & 2,65 \\
\hline Stand. Abw. & 0,17 & 0,62 \\
\hline Varianz & 0,14 & 1,97 \\
\hline F= & \multicolumn{2}{|c|}{13,902} \\
\hline W= & \multicolumn{2}{|c|}{32} \\
\hline$p=$ & $<0,0001$ \\
\hline
\end{tabular}

Tab. 72. Willendorf II, AH 6 und 8: Vergleich der SchlagflächenrestDicken (in $\mathrm{mm}$ ) zwischen allen vollständig und proximal erhaltenen Klingen und Lamellen (Wilcoxon-Mann-Whitney-Test).

Sticheln als Lamellenkerne anhand des Vorkommens einer Stichellamelle nachgewiesen. Diese Abbaustrategie konnte für AH 8 nicht dokumentiert werden.

Im Gegensatz zu AH 6 finden sich im Inventar von AH 8 Kerne und erlauben daher Aussagen zur Organisation der letzten Phase des Abbaus. Schlagunfälle stellen am häufigsten die letzte Operation an den vorwiegend stark reduzierten Kernen dar. Die verworfenen Kerne verweisen zusätzlich auf das Bestreben nach unidirektionalem, volumetrischen Abbau, da nur gegebenenfalls, wenn Schlagunfälle die Abbaufläche für das Ablösen einer nächsten Generation von Zielprodukten dermaßen unbrauchbar machten, weitere Schlag- bzw. Abbauflächen angelegt wurden.

\subsubsection{Werkzeugherstellung}

Insgesamt umfasst das Inventar aus AH 6 einen Werkzeuganteil von $7,8 \%$ und jenes aus $\mathrm{AH} 8$ einen von $21,2 \%$, womit sich ein wesentlich höherer Anteil an modifizierten Grundformen in AH 8 abzeichnet (Chi²-Test, $\mathrm{p}=0,0035)$ (Tab. 73). 


\begin{tabular}{|l|c|c|c|}
\hline & Modifiziert & Unmodifiziert & Gesamt \\
\hline AH 6 & 8 & 94 & 102 \\
\hline AH 8 & 40 & 149 & 189 \\
\hline \multicolumn{3}{|c|}{ Chi' $^{2}$-Test } \\
\hline F= & 1 \\
\hline Chi $^{2}=$ & 8,5344 \\
\hline$p=$ & 0,0034849 \\
\hline Fisher exact $p=$ & 0,0028999 \\
\hline
\end{tabular}

Tab. 73. Willendorf II, AH 6 und 8: Vergleich der Anteile an modifizierten und unmodifizierten Grundformen (Chi²-Test).

Außerdem wurde unter den Werkzeugen von AH 8 eine größere Typenvielfalt festgestellt, was aber auch ein Effekt der Anzahl der Steinartefakte in den beiden Inventaren sein könnte. Beide Schichten weisen jedoch Stichel, Kantenretuschen und Kratzer auf. In AH 8 treten zusätzlich noch Ausgesplitterte Stücke, Endretuschen, Spitzklingen sowie ein Bohrer und ein gezähntes Stück auf. Im Werkzeugbestand von $\mathrm{AH} 6$ hingegen stechen die Rückenelemente heraus, die in AH 8 zur Gänze fehlen. In AH 8 konnte mit Ausnahme der Spitzklingen keine Selektion von bestimmten Grundformen für bestimmte Werkzeugtypen beobachtet werden. In AH 6 allerdings wählten die SteinschlägerInnen sehr wohl exklusiv Lamellen für die Weiterverarbeitung zu rückengestumpften Stücken aus.

Das Werkzeugspektrum von AH 8 setzt sich ausschließlich aus Werkzeugen wie z. B. Kratzern, Sticheln, Ausgesplitterten Stücken, die für „häusliche“ Tätigkeiten eingesetzt wurden (domestic tools) zusammen, während hingegen in AH 6 die Rückenelemente eindeutig den Beleg für Projektile, die höchstwahrscheinlich für die Jagd verwendet wurden, darstellen.

\subsubsection{Technoökonomische Aspekte}

Der Unterschied in Bezug auf die genutzten Anteile an lokalen und nicht-lokalen Rohmaterialien zwischen AH 6 und AH 8 sticht heraus. Einhergehend zeigt sich in AH 6 der Bedarf nach diesen ortsfremden, hochwertigen Rohmaterialien, um regelmäßige und standardisierte Lamellen für die Modifikation zu Rückenelementen zu produzieren. Dieser weitläufig im Gravettien beobachtete Trend ${ }^{180}$ konnte jedoch in AH 8 nicht festgestellt werden. In AH 8 fehlen die Rückenelemente komplett und es dominieren Werkzeuge für „häusliche“ Tätigkeiten. Des Weiteren kommen die

180 Morala, Turq 1991. - O’Farrell 2000. - Floss, Kieselbach 2004. - Moreau 2009. - Moreau 2012. nicht-lokalen Rohmaterialien in AH 6 ausschließlich ohne natürliche Oberflächen oder mit stark reduzierter Bedeckung mit natürlichen Oberflächen vor, womit von einem intensiveren Abbau und von einer Durchführung der initialen Phase der Abbaukette außerhalb der Fundstelle ausgegangen wird. Doch liegen Absplisse und unretuschierte Grundformen aus nicht-lokalem Rohmaterial vor, was die Realisierung von späteren Phasen der Abbaukette vor Ort bestätigt. In AH 8 trifft dies auf den geringfügig präsenten baltischen Feuerstein ebenso zu, allerdings nicht auf den ebenfalls ortsfremden Jaspis, von welchem sehr wohl primäre Produkte vorhanden sind. Von den dominierenden, lokalen Rohmaterialien in AH 8 belegen Primärabschläge, Trümmer, Kerne sowie Kerntrümmer die Bewerkstelligung der vollständigen Abbaukette an der Fundstelle und außerdem, dass qualitativ hochwertige Ressourcen lokal zugänglich waren. ${ }^{181}$

Diese Diskrepanz zwischen den beiden $\mathrm{AH}$ in Bezug auf die Technoökonomie kann nicht durch die Erreichbarkeit und Zugänglichkeit der Rohmaterialquellen erklärt werden, da die generelle Topografie der Region über die letzten Jahrzehntausende weitgehend unverändert blieb. ${ }^{182}$ Vielmehr dürften Unterschiede in der Mobilität und Landnutzung, die starken Einfluss auf die Versorgungsstrategien (provisioning strategies) bzw. die Kombination an Versorgungsstrategien haben, ${ }^{183}$ in den beiden Horizonten vorliegen. Vorwiegend dürften zwar die SteinschlägerInnen sowohl von AH 6 als auch von AH 8 die Strategie der Versorgung der Plätze (provisioning of places) verfolgt haben. Diese Versorgung erfordert, dass Rohmaterialien von der Beschaffungslokalität an den bestimmten zu versorgenden Ort gebracht und dort angehäuft werden, um, wenn der Bedarf besteht, zur Nutzung zur Verfügung zu stehen. ${ }^{184}$ Allerdings dürften Unterschiede bei den geplanten Aktivitäten vor Ort, der relativen Besiedlungsdauer und der geografischen Reichweite des Sammelterritoriums bestanden haben. ${ }^{185}$ Der Unterschied im Werkzeugbestand deutet auf unterschiedliche Aktivitäten hin. Die hohe Komponente an lokalen Rohmaterialien in AH 8 deutet unter anderem an, dass die Aufenthalte länger waren und vermehrt auf Knollen aus den Donauschottern, obwohl bei diesen die Qualität wegen der Heterogenität weniger abschätzbar war, zurückgegriffen wurde, um Materialdefizit $\mathrm{zu}$ vermeiden. ${ }^{186}$

\footnotetext{
181 Vgl. Roth, Dibble 1998.

182 Moreau, Brandl, Nigst 2016, 86.

183 KuHN 2004, 433.

184 Vgl. Kuhn 1995. - KuHn 2004.

185 Siehe Moreau, Brandl, Nigst 2016.

186 Moreau, Brande, Nigst 2016.
} 
Zuletzt könnte der verstärkte Import von vorpräparierten, ortsfremden Rohmaterialien darauf verweisen, dass die Menschen von AH 6 ein größeres Sammelterritorium hatten als jene aus AH 8, weil sie entweder mobiler oder weitläufiger vernetzt waren.

\subsubsection{Implikationen für die chrono-kulturelle Gliederung}

Die beiden AH 6 und 8 der Altgrabungen in Willendorf II wurden einerseits von Svoboda et al. ${ }^{187}$ zusammenfassend dem Pavlovien zugeordnet und andererseits von Otte ${ }^{188}$ in seiner Gliederung des mitteleuropäischen Gravettien zwei unterschiedlichen Stadien („Stade 1“ und „Stade 2“) zugewiesen. Svoboda et al. ${ }^{189}$ argumentierten aufgrund der typologischen Zusammensetzung, unter anderem dem Anstieg von kantenretuschierten Klingen, und dem Vorherrschen von Radiolarit bei den Rohmaterialien, dass die AH 6 bis 8 zusammengehörig sind. Otte ${ }^{190}$ führte typologische und technologische Merkmale als eindeutige Unterschiede an. Sein „Stade 1“ umfasst geometrische Mikrolithen, Gravette- sowie Mikrogravettespitzen und fléchette an Lamellen gefertigt, während sich die Inventare des „Stade 2“ durch eine vermehrte Klingenproduktion und die Herstellung von Spitzklingen auszeichnen.

Die hier vorgelegten techno-typologischen Ergebnisse von AH 8 stimmen mit der Definition des „Stade 2“ von Otte ${ }^{191}$ überein und auch die Zuweisung zum (entwickelten) Pavlovien von Svoboda et al. ${ }^{192}$ trifft zu. Das Werkzeugspektrum von AH 8 beinhaltet verschiedene Typen, die diagnostisch für das (entwickelte) Pavlovien sind. ${ }^{193}$ Spitzklingen treten als besonders charakteristische Vertreter auf. Außerdem kommt ein hoher Anteil von Ausgesplitterten Stücken vor. Zusätzlich wurden auch Stichel, Kratzer, ein Bohrer mit Kantenretusche und ein gezähntes Stück festgestellt. Einzig in Bezug auf das Rohmaterial konnte das Vorherrschen von Radiolarit nicht bestätigt werden. Allerdings stimmt die häufige Nutzung von Quarzit mit den Beobachtungen von Otte ${ }^{194}$ überein.

Die Resultate zu AH 6 zeigen einige auffällige Unterschiede zu AH 8. Daher wird im Folgenden ein Vergleich

187 SvOBODA, LOŽEK, VLČEK 1996.

188 ОTTE 1981. - ОтTE 1991.

189 SvoвodA, LožeK, VLČEK 1996, 138.

190 OTTE 1981.

191 OTTE 1981.

192 Svoboda, LožEK, VLČEK 1996.

193 Valoch 1981. - Svoboda 1994a. - Svoboda 1996. - Moreau

2009. - Svoboda 2012.

194 OTTE 1981. mit den diagnostischen technologischen Hauptmerkmalen einerseits von AH 5 und andererseits von AH 6 der Altgrabungen vorgenommen. Es muss jedoch an dieser Stelle darauf hingewiesen werden, dass die geringe Stückzahl beider Inventare einen limitierenden Faktor für die Repräsentativität darstellt und die Unterschiede eventuell mit der Funktion der Fundplatzbegehungen oder zumindest der durch die Ausgrabungen erfassten Fundplatzbereiche zusammenhängen könnten.

AH 5 wurde von Otte ${ }^{195}$ dem „Stade 1“ zugewiesen und von Moreau ${ }^{196}$ neu analysiert. Die am häufigsten verwendeten Rohmaterialien in AH 5 sind mit 66,6 \% lokale Gesteine wie alpiner Hornstein, Radiolarit, Quarzit und Quarz. Feuerstein umfasst 15,8\% und bei weiteren 5,5\% konnte nicht eindeutig festgestellt werden, ob es sich um Feuerstein oder Mährischen Jurahornstein handelt. ${ }^{197}$ In AH 6 machen alpiner Hornstein und Radiolarit zusammen 65,7 \% aus und Feuerstein 27,5\%.

Der Kernabbau von AH 5 erfolgte unidirektional von volumetrischen, prismatischen Kernen durch den Einsatz von sowohl dem direkten, weichen, mineralischen als auch dem direkten, organischen Schlag und zielte auf die Produktion von Klingen und Lamellen ab, wobei ein Kontinuum in der Abbaukette von Klingen bzw. kleinen Klingen zu Lamellen vorherrscht. ${ }^{198}$ Die Initialisierung wurde entweder mit Kernkantengrundformen oder mit primären Klingen eingeleitet. Die Mehrzahl der Kerne verfügt über eine Schlag- sowie Abbaufläche und diejenigen Kerne mit einer zweiten, gegenüberliegenden Schlagfläche belegen, dass diese zur Zurichtung der Abbaufläche dienten und die Abbaulogik trotzdem unidirektional war. Außerdem wurde eine unabhängige Abbaustrategie, bei welcher Stichel als Lamellenkerne genutzt wurden, festgestellt. In AH 6 lief der Kernabbau wahrscheinlich sehr ähnlich ab, allerdings liegen keine Kerne zum Vergleich vor und die Klingenkomponente ist sehr gering.

Die Grundformen mit Kantenretuschen (29\%) dominieren das Werkzeugspektrum von AH 5 und die als Projektile genutzten Rückenelemente (20,5\%) sind am zweithäufigsten vertreten. ${ }^{199}$ Des Weiteren kommen verschiedenste Stichel- sowie Kratzertypen, Spitzklingen, Kombinationsgeräte aus Kratzer-Stichel und Endretuschen vor. Zuletzt

195 ОTTE 1981. - ОтTЕ 1991.

196 Moreau 2009. - Moreau 2012. - Moreau, Brandl, Nigst 2016.

197 Moreau, Brandl, Nigst 2016.

198 Moreau 2009, 271-283.

199 Moreau, Brandl, Nigst 2016, 89-90. 
wurden noch zu geringen Anteilen Schaber, Ausgesplitterte Stücke und Bohrer dokumentiert. Der Werkzeugbestand von AH 6 beinhaltet zu gleichen Teilen die vier in AH 5 am stärksten vertretenen Werkzeugtypen.

AH 6 der Altgrabungen weist ein Steininventar von 255 Stücken auf. ${ }^{200}$ Allerdings muss davon ausgegangen werden, dass dies nur einen ausgewählten Teil des gegrabenen Gesamtinventars darstellt, da im Naturhistorischen Museum Wien kürzlich weitere Holzkisten mit neuem und nicht-inventarisiertem lithischen Fundmaterial der Altgrabungen aufgefunden wurden. ${ }^{201}$ Diese Schicht wurde von Otte ${ }^{202}$ ebenfalls dem „Stade 1“ zugeordnet, wobei er dazu schreibt: „L'ensemble du niveau 6 , très proche encore du niveau 5 , annonce les tendances de la phase médiane (niveaux 7 et 8): réduction des fléchettes, microlithes et pièces à dos et augmentation des lames retouchées. “203 Das am häufigsten genutzte Rohmaterial in AH 6 der Altgrabungen stellt Feuerstein mit 39,5\% dar, gefolgt von Hornstein mit $25 \%$. Quarzit umfasst 12,3\% des Rohmaterialspektrums, während Radiolarit 11,8\% ausmacht. Außer Quarzit, der mit nur einem Stück vertreten ist, sind dies auch die am meisten genutzten Rohmaterialien in $\mathrm{AH}$ 6, wobei aber der alpine Hornstein dominiert.

Im Inventar von AH 6 der Altgrabungen liegen 24 Kerne vor. ${ }^{204}$ Bei 67 \% der Kerne wurden Gesteinsblöcke oder Gerölle als Ausgangsform genutzt und diese waren ausschließlich für die Lamellenproduktion bestimmt. Geringfügig dienten auch dicke Abschläge als Ausgangsformen. Die SteinschlägerInnen wählten hauptsächlich längliche Gesteinsblöcke oder flache Gerölle aus, um direkt ohne intensive Präparation den Abbau beginnen zu können. Daher wurden auch selten Kernkanten angelegt. Pro Kern wurden im Durchschnitt 5,38 Klingen bzw. Lamellen produziert. Die Mehrzahl der Kerne (54\%) wurde unidirektional abgebaut, wobei Kerne mit einer zweiten Schlagfläche entweder gegenüberliegend (21\%) oder rechtwinkelig (8\%) zur ersten auftreten. Die Klingen und Lamellen weisen schmale oder punktförmige Schlagflächenreste, diffuse Bulben und dorsale Reduktion auf, womit starke Indizien für den direkten, weichen Schlag vorliegen. Die Zielprodukte sind

200 ОтTЕ $1981,73$.

201 Nigst 2004.

202 ОTTE 1981. - ОтTЕ 1991.

203 Отте 1981, 88. „Das Inventar von AH 6, immer noch sehr nah an AH 5, kündigt Tendenzen der mittleren Phase an (Stufen 7 und 8): Abnahme von fléchettes, Mikrolithen und rückengestumpften Elementen und Anstieg der retuschierten Klingen." [Übersetzung V. C. Schmid].

204 OTTE 1981. morphologisch regelmäßig, schmal und geradlinig. Die durchschnittliche Länge der Klingen bzw. Lamellen liegt bei $50,4 \mathrm{~mm}$ und die durchschnittliche Breite bei 15,2 $\mathrm{mm}$. Klingen und Lamellen umfassen $83 \%$ der Grundformen und weitere $12 \%$ entfallen auf Stichellamellen. Abschläge sind mit nur $5 \%$ vertreten. Aufgrund der dorsalen Negative der Grundformen von AH 6 kann bestätigt werden, dass ebenso eine unidirektionale Abbaustrategie im Vordergrund stand, aber eben auch Grundformen mit bidirektionalen sowie gleichgerichtet und quer verlaufenden Negativen vorkommen. Kernkantengrundformen sind geringfügig vertreten. Alle Klingen und Lamellen weisen dorsale Reduktion sowie schmale und dünne Schlagflächenreste auf und bezeugen den Einsatz entweder des direkten, weichen, mineralischen oder des direkten, organischen Schlages. Die Mittelwerte der Länge (21,6 mm) und Breite $(8,23 \mathrm{~mm})$ bei den Klingen bzw. Lamellen von AH 6 fallen deutlich kürzer und schmäler gegenüber jenen der Altgrabungen aus. Das Grundformenspektrum umfasst 45,2 \% Klingen bzw. Lamellen sowie 42,2 \% Abschläge und damit einen größeren Anteil an Abschlägen als das Inventar der Altgrabungen. Eine Stichellamelle ist auch unter den Grundformen von AH 6 verzeichnet.

Bei 27,5 \% der Steinartefakte aus AH 6 der Altgrabungen handelt es sich um Werkzeuge. ${ }^{205}$ Retuschierte Klingen dominieren mit 27,1\%, gefolgt von Sticheln mit 21,4\% und Kratzern mit 12,9 \%. Rückengestumpfte Elemente, darunter vorwiegend Gravette- und Mikrogravettespitzen, sind mit 5,7 \% des Werkzeugbestands neben sogenannten pointes à retouches plates und informalen Werkzeugen am viertstärksten vertreten. Fléchettes und Mikrolithen kommen nicht vor. Der Werkzeuganteil von 7,8 \% fällt in AH 6 geringer als bei den Altgrabungen aus. Das Werkzeugspektrum stimmt jedoch mit den Altgrabungen überein.

Der Vergleich von AH 6 (Ausgrabung 1993) mit AH 5 der Altgrabungen auf der einen Seite und mit AH 6 der Altgrabungen auf der anderen Seite verweist auf schlagende Ähnlichkeiten, welche dafürsprechen, dass AH 6 eher $\mathrm{zu}$ „Stade 1“ einhergehend mit der Einteilung von Otte ${ }^{206}$ zu zählen ist. AH 6 würde demnach nicht in die Phase des Pavlovien fallen, sondern dem frühen Gravettien angehören wie AH 5 von Willendorf II, des Weiteren AH I von Geißenklösterle, AH IIb, IIc sowie IIcf von Hohle Fels, Weinberghöhlen (alle Deutschland) und Molodova V, AH 9 und 10 (Ukraine). ${ }^{207}$ Die Datierungen von AH 6 stehen dieser

\footnotetext{
205 ОTTE 1981.

206 ОтT' 1981.

207 Moreau 2010.
} 
Interpretation nicht entgegen. Allerdings steht diese Interpretation unter dem Vorbehalt der geringen Stückzahl. ${ }^{208}$

Die technologischen Innovationen sowie die Mobilitätsstrategien des Pavlovien, welche Lagerplatzbelegung, Landschaftsnutzung und Gruppenvernetzung involvieren, dürften in AH 8 etabliert gewesen sein, während die SteinschlägerInnen von AH 6 eher noch die Verhaltensmuster des frühen Gravettien aufweisen und eventuell, wie Otte ${ }^{209}$ es beschreibt, ein erstes Aufflammen der Neuerungen andeuten.

\section{Danksagung}

Wir danken ganz herzlich Gerhard Trnka, Universität Wien, für seine langjährige Unterstützung, gute Diskussionen und Zurverfügungstellung von fotografischer Dokumentation der Ausgrabung 1993. Außerdem gilt unser Dank Frank Brodbeck, Eberhard Karls Universität Tübingen, für die Zeichnungen. Wir möchten uns abschließend bei zwei anonymen Gutachtern bedanken, die dazu beigetragen haben, das Manuskript zu verbessern.

P. R. Nigsts Forschungen wurden durch Fördermittel von folgenden Institutionen finanziert: Leakey Foundation, Europäische Kommission (EC FP7 Career Integration Grant, Projekt „NEMO-ADAP“, Nr. 322261), Sir Isaac Newton Trust, British Academy (British Academy/Leverhulme Small Grant), Max-Planck-Gesellschaft, McDonald Institute for Archaeological Research, University of Cambridge, Universität Wien, Hugo Obermaier Preis 2006 und Hochschuljubiläumsfonds der Stadt Wien. M. D. Boschs Forschungen wurden durch Fördermittel von der Europäischen Kommission (EC H2020 Marie Skłodowska Curie program EF, Projekt „EU-Beads", Nr. 656325) finanziert.

\section{Beiträge der Autorinnen und Autoren}

Viola C. Schmid: Studienkonzept, technologische Analyse des lithischen Materials, Rohmaterialanalyse des lithischen Materials, statistische Auswertung, Verfassen des Manuskripts

Marjolein D. Bosch: Archäozoologische Untersuchung

Michael Brandl: Rohmaterialanalyse des lithischen Materials

Michael Götzinger: Rohmaterialanalyse des lithischen Materials Philip R. Nigst: Studienkonzept, statistische Auswertung, Verfassen des Manuskripts

\section{Literatur}

\section{ADLER 2002}

D. S. Adler, Late Middle Palaeolithic Patterns of Lithic Reduction, Mobility, and Land Use in the Southern Caucasus. Dissertation, Harvard University, Cambridge 2002.

Angeli 1989

W. Angeli, Die Venus von Willendorf. Wien 1989.

\section{ANTL-Weiser 1999}

W. Antl-Weiser, Paläolithischer Schmuck von der Gravettienfundstelle Grub/Kranawetberg bei Stillfried, Niederösterreich, Annalen des Naturhistorischen Museums in Wien 101 A, 1999, 23-41.

208 Haesaerts et al. 1996.

209 ОтTе 1981, 88.
AnTL-Weiser 2000-2001

W. AntL-Weiser, Die Auffindung der Venus von Willendorf - eine unendliche Geschichte, Mitteilungen der Anthropologischen Gesellschaft in Wien 130-131, 2000-2001, 40-57.

AntL-Weiser 2005

W. ANTL-Weiser, L'industrie en os et en ivoire du site gravettien à Grub/Kranawetberg près de Stillfried (Note préliminaire). In: D. Vialou, J. Renault-Misokovsky, M. Patou-Mathis (Hrsg.), Comportements des hommes du Paléolithique moyen et supérieur en Europe: territoires et milieux. Actes du Colloque du G.D.R. 1945 du CNRS, Paris, 8-10 janvier 2003. Études et Recherches Archéologiques de l'Université de Liège 111, Lüttich 2005, 511-58.

ANTL-WeISER 2008a

W. ANTL-Weiser, Die Frau von W.: Die Venus von Willendorf, ihre Zeit und die Geschichte(n) um ihre Auffindung. Veröffentlichungen der Prähistorischen Abteilung 1, Wien 2008.

ANTL-WEISER 2008b

W. Antr-Weiser, Die Venus von Willendorf - 100 Jahre danach, Mitteilungen der Anthropologischen Gesellschaft in Wien 138, 2008, 1-21.

AnTL-Weiser 2013

W. AntL-Weiser, The inventories of archaeological horizons 4 and 3 and the loess section of Grub/Kranawetberg, a Gravettian site in Lower Austria, E\&G Quaternary Science Journal 62, 2013, $120-126$.

AnTL-Weiser et al. 2010

W. Antl-Weiser, F. A. Fladerer, P. R. Nigst, A. Verpoorte, Grub/Kranawetberg (Lower Austria) - Insights into a Gravettian micro-region in Eastern Austria. In: C. Neugebauer-MAResCh, L. R. Owen (Hrsg.), New Aspects of the Central and Eastern European Upper Palaeolithic - Methods, Chronology, Technology and Subsistence. Mitteilungen der Prähistorischen Kommission 72, Wien 2010, 231-244.

AntL-Weiser, Bosch 2015

W. AnTL-Weiser, M. D. Bosch, The use of ivory at the Gravettian site Grub/Kranawetberg, Lower Austria, Anthropologie LIII/1-2, 2015, 233-244.

Aubry, Fontugne, Moura 1997

T. Aubry, M. Fontugne, M.-H. Moura, Les occupations de la grotte de Buraca Grande depuis le Paléolithique supérieur et les apports de la séquence holocène à l'étude de la transition Mésolithique/Néolithique au Portugal, Bulletin de la Société Préhistorique Française 94, 1997, 182-190.

Auffermann et al. 1990

B. Auffermann, W. Burkert, J. Hahn, C. Pasda, U. Simon, Ein Merkmalsystem zur Auswertung von Steinartefaktinventaren, Archäologisches Korrespondenzblatt 20, 1990, 259-268.

Bar-Yosef, Van Peer 2009

O. Bar-Yosef, P. Van Peer, The chaine opératoire approach in Middle Paleolithic archaeology, Current Anthropology 50/1, 2009, 103-131.

BAYER 1928

J. BAYER, Das zeitliche und kulturelle Verhältnis zwischen den Kulturen des Schmalklingenkulturkreises während des Diluviums in Europa, Eiszeit und Urgeschichte 5, 1928, 9-23.

BAYER 1929

J. BAYER, Die Olschewakultur, Eiszeit und Urgeschichte 6, 1929, $83-100$. 
BAYER 1930

J. BAYER, Die Venus von Willendorf, Eiszeit und Urgeschichte 7, 1930, 48-54.

BinSTEIner, Eisner, ANTL-Weiser 2008

A. Binsteiner, R. Eisner, W. Antl-Weiser, Die Rohstoffanalyse der Silexartefakte von Willendorf II, Mitteilungen der Anthropologischen Gesellschaft in Wien 138, 2008, 63-78.

BORDES, LENOBLE 2002

J.-G. Bordes, A. Lenoble, La „lamelle Caminade“: un nouvel outil lithique aurignacien? Bulletin de la Société Préhistorique Française 99, 2002, 735-749.

Borglio, Laplace 1966

A. Borglio, G. Laplace, Études de typologie analytique des complexes leptolithiques de l'Europe centrale II - Les complexes gravettiens de la Basse Autriche: Willendorf II, Rivista di Scienze Preistoriche 21, 1966, 303-364.

Bosch 2012

M. D. Bosch, Human-mammoth dynamics in the Mid-Upper Palaeolithic of the Middle Danube Region, Quaternary International 276-277, 2012, 170-182.

Bosch et al. 2012

M. D. Bosch, P. R. Nigst, F. A. Fladerer, W. Antl-Weiser, Humans, bones and fire: zooarchaeological, taphonomic, and spatial analyses of a Gravettian mammoth bone accumulation at Grub-Kranawetberg (Austria), Quaternary International 252, 2012, 109-121.

BrandL et al. 2014

M. Brandl, C. Hauzenberger, W. Postl, M. M. Martinez, P. Filzmoser, G. Trnka, Radiolarite studies at Krems-Wachtberg (Lower Austria): northern Alpine vs. Carpathian lithic resources, Quaternary International 351, 2014, 146-162.

BRANDTNER 1956-1959

F. BRANDTNER, Die geologisch-stratigraphische Position der Kulturschichten von Willendorf i. d. Wachau, Niederösterreich. In: F. Felgenhauer (Hrsg.), Willendorf in der Wachau: Monographie der Paläolith-Fundstellen I-VII. Mitteilungen der Prähistorischen Kommission 8-9, Teil 1, Wien 1956-1959, 172-198.

Chernysh 1961

A. P. Chernysh, Paleolitichna Stoyanka Molodove V. Kiev 1961.

Cziesla 1990

E. Cziesla, On refitting of stone artefacts. In: E. Cziesla, S. EicKhoff, N. Arts, D. Winter (Hrsg.), The Big Puzzle. International Symposium on Refitting Stone Artefacts at Monrepos, Neuwied 1987. Studies in Modern Archaeology 1, Bonn 1990, 9-44.

D’ANgelo, Mussi 2005

E. D’Angelo, M. Mussi, Galets et lamelles de L'Aurignacien du Latium (Italie centrale): le cas de la grotta Barbara. In: F. LE Brun-Ricalens, J.-G. Bordes, F. Bon (Hrsg.), Productions lamellaires attribuées à l'Aurignacien: chaînes opératoires et perspectives technoculturelles. Actes du XIV e congrès de l'Union Internationale des Sciences Préhistoriques et Protohistoriques, Liège, 2-8 septembre 2001. ArchéoLogiques 1, Luxemburg 2005, 313-322.

Damblon, Haesaerts, Van Der Plicht 1996

F. Damblon, P. Haesaerts, J. Van Der Plicht, New datings and considerations on the chronology of Upper Paleolithic sites in the Great Eurasiatic Plain, Préhistoire Européenne 9, 1996, 177-231.
De Loecker, Schlanger 2004

D. De Loecker, N. Schlanger, Analysing Middle Palaeolithic flint assemblages: the system used for studying the flint artefacts at Maastricht-Belvédère (The Netherlands). In: D. De Loecker (Hrsg.), The Saalian Archaeological Record at Maastricht-Belvédère (The Netherlands). Analecta Praehistorica Leidensia 35-36, Leiden 2004, 303-345.

De Sonneville-Bordes 1960

D. DE SonNeville-Bordes, Le Paléolithique supérieur en Périgord. Bordeaux 1960.

Dibble, Pelcin 1995

H. L. Dibble, A. W. Pelcin, The effect of hammer mass and velocity on flake mass, Journal of Archaeological Science 22, 1995, 429-439.

Dibble, ReZeK 2009

H. L. Dibble, Z. Rezek, Introducing a new experimental design for controlled studies of flake formation: results for exterior platform angle, platform depth, angle blow, velocity, and force, Journal of Archaeological Science 36, 2009, 1945-1954.

Dibble, WhitTaker, 1981

H. L. Dibble, J. C. Whittaker, New experimental evidence on the relation between percussion flaking and flake variation, Journal of Archaeological Science 8, 1981, 283-296.

Domanski, Webb 1992

M. Domanski, J. Weвb, Effect of heat treatment on siliceous rocks used in prehistoric lithic technology, Journal of Archaeological Science 19, 1992, 601-614.

Domanski, Webb, Boland 1994

M. Domanski, J. A. Webb, J. Boland, Mechanical properties of stone artefact materials and the effect of heat treatment, Archaeometry 36, 1994, 177-208.

EINWÖGERER 2000

T. EINWÖgERER, Die jungpaläolithische Station auf dem Wachtberg in Krems, NÖ. Mitteilungen der Prähistorischen Kommission 34, Wien 2000.

EINWÖGERER 2010

T. EInwÖgerer, Excavations at the Krems-Wachtberg site and the discovery of the infant burials. In: C. Neugebauer-Maresch, L. R. Owen (Hrsg.), New Aspects of the Central and Eastern European Upper Palaeolithic - Methods, Chronology, Technology and Subsistence. Mitteilungen der Prähistorischen Kommission 72, Wien 2010, 273-284.

EINWÖGERER et al. 2006

T. Einwögerer, H. Friesinger, M. Händel, C. Neugebauer-MaResch, U. Simon, M. Teschler-Nicola, Upper Palaeolithic infant burials, Nature 444, 2006, 285.

FARBSTEIN 2011a

R. FARBSTEIN, Technologies of art: a critical reassessment of Pavlovian art and society, using chaîne opératoire method and theory, Current Anthropology 52/3, 2011, 401-432.

FARBSTEIN 2011b

R. FARbSTEIn, The significance of social gestures and technologies of embellishment in Paleolithic portable art, Journal of Archaeological Method and Theory 18, 2011, 125-146.

Farbstein, Davies 2017

R. Farbstein, W. Davies, Palaeolithic ceramic technology: the artistic origins and impacts of a technological innovation, Quaternary International 441, 2017, 3-11. 
Felgenhauer 1951

F. Felgenhauer, Aggsbach, ein Fundplatz des späten Paläolithikums in Niederösterreich. Mitteilungen der Prähistorischen Kommission 5, Wien 1951.

FELGENHAUER 1956-1959

F. Felgenhauer, Willendorf in der Wachau: Monographie der Paläolith-Fundstellen I-VII. Mitteilungen der Prähistorischen Kommission 8-9, Teil 1, Wien 1956-1959.

FeLgenHAUER 1995-1996

F. Felgenhauer, Aggsbachien-Gravettien-Pavlovien: Zur Frage nomenklatorischer Prioritäten in der Urgeschichtsforschung, Mitteilungen der Anthropologischen Gesellschaft in Wien 125126, 1995-1996, 249-257.

FLADERER 2001

F. A. FLADERER, Die Faunareste vom jungpaläolithischen Lagerplatz Krems-Wachtberg, Ausgrabung 1930: Jagdwild und Tierkörpernutzung an der Donau vor 27.000 Jahren. Mitteilungen der Prähistorischen Kommission 39, Wien 2001.

Fladerer, Salcher-Jedrasiak, Händel 2014

F. A. Fladerer, T. A. Salcher-Jedrasiak, M. Händel, Hearthside bone assemblages within the $27 \mathrm{ka} \mathrm{BP}$ Krems-Wachtberg settlement: fired ribs and the mammoth bone-grease hypothesis, Quaternary International 351, 2014, 115-133.

Floss, Kieselbach 2004

H. Floss, P. Kieselbach, The Danube Corridor after 29.000 BP: new results on raw material procurement patterns in the Gravettian of Southwestern Germany, Mitteilungen der Gesellschaft für Urgeschichte 13, 2004, 61-78.

FRANK, RABEDER 1994

C. Frank, G. Rabeder, Neue ökologische Daten aus dem Lössprofil von Willendorf in der Wachau, Archaeologia Austriaca 52, 1994, $59-65$.

GÖTZINGER 2006

M. A. GÖTZINGER, Überblick zu den verfügbaren Steinrohstoffen in Ostösterreich. Beitrag in: I. Mateiciucová, G. Trnka, M. A. GÖTZINGER, Zur Rohstoffverteilung und -verfügbarkeit in der Lengyel-Kultur, Archäologie Österreichs 17/2, 2006, $85-88$.

HäNDEL et al. 2009

M. Händel, U. Simon, T. Einwögerer, C. Neugebauer-MaRESCH, New excavations at Krems-Wachtberg: approaching a well-preserved Gravettian settlement site in the Middle Danube Region, Quartär 56, 2009, 187-196.

HAESAERTS 1990

P. Haesaerts, Nouvelles recherches au gisement de Willendorf (Basse Autriche), Bulletin de l'Institut Royal des Sciences Naturelles de Belgique, Sciences de la Terre 60, 1990, 203-218.

Haesaerts, Teyssandier 2003

P. Haesaerts, N. Teyssandier, The early Upper Paleolithic occupations of Willendorf II (Lower Austria): a contribution to the chronostratigraphic and cultural context of the beginning of the Upper Paleolithic in Central Europe. In: J. ZiLhão, F. D’Errico (Hrsg.), The Chronology of the Aurignacian and the Transition Technocomplexes. Dating, Stratigraphies and Cultural Implications. Trabalhos de Arqueologia 33, Lissabon 2003, 133-151.

Haesaerts et al. 1996

P. Haesaerts, F. Damblon, M. Bachner, G. Trnka, Revised stratigraphy and chronology of the Willendorf II sequence, Lower Austria, Archaeologia Austriaca 80, 1996, 25-42.
Haesaerts et al. 2003

P. Haesaerts, I. Borziak, V. Chirica, F. Damblon, L. KoulaKovska, J. VAn Der Plicht, The east Carpathian loess record: a reference for the middle and late pleniglacial stratigraphy in central Europe / La séquence loessique du domaine est-carpatique: une référence pour le Pléniglaciaire moyen et supérieur d’Europe centrale, Quaternaire 14, 2003, 163-188.

HAEsAerts et al. 2009

P. Haesaerts, I. Borziac, V. P. Chekha, V. Chirica, F. Damblon, N. I. Drozdov, L. A. Orlova, S. Pirson, J. Van Der Plicht, Climatic signature and radiocarbon chronology of middle and late pleniglacial loess from Eurasia: comparison with the marine and Greenland records, Radiocarbon 51, 2009, 301-318.

\section{HAHN 1969}

J. HAHN, Gravettien-Freilandstationen im Rheinland: Mainz-Linsenberg, Koblenz-Metternich und Rhens, Bonner Jahrbücher $169,1969,44-87$.

HAHN 1977

J. HaHn, Aurignacien: Das ältere Jungpaläolithikum in Mittel- und Osteuropa. Köln - Wien 1977.

HAHN 1993

J. Hahn, Erkennen und Bestimmen von Stein- und Knochenartefakten: Einführung in die Artefaktmorphologie. Mitteilungsblatt der Archaeologica Venatoria 10, Tübingen 1993.

Hammer, Harper, Ryan 2001

Ø. Hammer, D. A. T. Harper, P. D. Ryan, PAST: Paleontological statistics software package for education and data analysis, Palaeontologia Electronica 4, 2001, 9.

HeNshiLwOod et al. 2011

C. S. Henshilwood, F. D’Errico, K. L. Van Niekerk, Y. Coquinot, Z. Jacobs, S.-E. Lauritzen, M. Menu, R. García-MoreNo, A 100,000-year-old ochre-processing workshop at Blombos Cave, South Africa, Science 334/6053, 2011, 219-222.

Higham et al. 2011

T. Higham, R. Jacobi, L. Basell, C. Bronk Ramsey, L. Chiotti, R. NesPoulet, Precision dating of the Palaeolithic: a new radiocarbon chronology for the Abri Pataud (France), a key Aurignacian sequence, Journal of Human Evolution 61, 2001, 549-563.

INIZAN et al. 1999

M.-L. Inizan, M. Reduron-Ballinger, H. Roche, J. Tixier, Technology of Knapped Stone. Nanterre 1999.

Klaric, Aubry, Walter 2002

L. Klaric, T. Aubry, B. Walter, Un noveau type d'armature en contexte gravettien et son mode de production sur les burins du Raysse (la Picardie, commune de Preuilly-sur-Claise, Indre-etLoire), Bulletin de la Société Préhistorique Française 99, 2002, 751-764.

KLÍMA 1959

B. KLÍma, Zur Problematik des Aurignacien und Gravettien in Mittel-Europa, Archaeologia Austriaca 26, 1959, 35-51.

KLÍma 1968

B. KLíma, Das Pavlovien in den Weinberghöhlen von Mauern, Quartär 19, 1968, 263-273.

KLÍ́ma 1995

B. KLÍMA, Dolní Vestonice II: Ein Mammutjägerplatz und seine Bestattungen. Études et Recherches Archéologiques de l'Université de Liège 73, Dolní Vestonice Studies 3, Lüttich 1995. 
KLÍMA 1997

B. Klíma, Bone industry, decorative objects, and art / Knochenindustrie, Zier- und Kunstgegenstände. In: J. SvoворA (Hrsg.), Pavlov I-Northwest: The Upper Paleolithic Burial and Settlement Context. Dolní Vestonice Studies 4, Brünn 1997, 227-286.

KozŁowsKi 1986

J. K. KozŁowski, The Gravettian in Central and Eastern Europe, Advances in World Archaeology 5, 1986, 131-200.

KROMER 1950

K. Kromer, J. Bayers „Willendorf II“-Grabung im Jahre 1913, Archaeologia Austriaca 5, 1950, 63-79.

KuHN 1995

S. L. Kuhn, Mousterian Lithic Technology: An Ecological Perspective. Princeton 1995.

KuHN 2004

S. L. Kunn, Upper Paleolithic raw material economies at Ücagizlı cave, Turkey, Journal of Anthropological Archaeology 23, 2004, 431-448.

Le Brun-Ricalens, Brou 2003

F. Le Brun-Ricalens, L. Brou, Burins carénés-nucléus à lamelles: identification d'une chaîne opératoire particulière à Thèmes (Yonne) et implications, Bulletin de la Société Préhistorique Française 100, 2003, 67-83.

Le BRun-Ricalens 2006

F. Le BRUN-Ricalens, Les pièces esquillées: état des connaissances après un siècle de reconnaissance, Paléo Revue d'archéologie préhistorique 18, 2006, 95-114.

LuCAS 2002

G. Lucas, A propos des burins du Raysse du Flageolet I (Dordogne, France), Paléo 14, 2002, 63-76.

Mason, Hather, Hillman 1994

S. L. R. Mason, J. G. Hather, G. C. Hillman, Preliminary investigation of the plant macro-remains from Dolní Věstonice II, and its implications for the role of plant foods in Palaeolithic and Mesolithic Europe, Antiquity 68, 1994, 48-57.

Mcpherron, Dibble 2002

S. P. Mcpherron, H. L. Dibble, Using Computers in Archaeology: A Practical Guide. New York 2002.

Morala, Turq 1991

A. Morala, A. Turq, Relations entre matières premières lithiques et technologie: l'exemple du Paléolithique entre Dordogne et Lot. In: 25 ans d'études technologiques et préhistoriques: bilan et perspectives. Actes des XI ${ }^{\mathrm{e}}$ Rencontres Internationales d'Archéologie et d'Histoire d'Antibes, 18-20 octobre 1990. Juan-lePins 1991, 159-168.

Moreau 2009

L. Moreau, Geißenklösterle-Das Gravettien der Schwäbischen Alb im europäischen Kontext. Tübingen 2009.

Moreau 2010

L. Moreau, Geißenklösterle: the Swabian Gravettian in its European context, Quartär 57, 2010, 79-93.

Moreau 2012

L. Moreau, Le Gravettien ancien d'Europe centrale revisité: mise au point et perspectives, l'Anthropologie 116, 2012, 609-638.

Moreau, Brandl, Nigst 2016

L. Moreau, M. Brandl, P. R. Nigst, Did prehistoric foragers behave in an economically irrational manner? Raw material availability and technological organisation at the early Gravettian site of Willendorf II (Austria), Quaternary International 406, 2016, 84-94.
Movius 1977

H. L. Movius, Excavation of the Abri Pataud, Les Eyzies (Dordogne): Stratigraphy. American School of Prehistoric Research Bulletin 31, Cambridge 1977.

Movius et al. 1968

H. L. Movius, N. C. David, H. M. Bricker, R. B. Clay, The Analysis of Certain Major Classes of Upper Palaeolithic Tools. American School of Prehistoric Research Bulletin 26, Cambridge 1968.

Neugebauer-Maresch 2008

C. Neugebauer-Maresch, Krems-Hundssteig - Mammutjägerlager der Eiszeit: Ein Nutzungsareal paläolithischer Jäger- und Sammler(innen) vor 41.000-27.000 Jahren. Mitteilungen der Prähistorischen Kommission 67, Wien 2008.

Nigst 2004

P. R. Nigst, „Neue“ alte Funde aus Willendorf II, Schicht 3, Archäologie Österreichs 15/1, 2004, 23-25.

Nigst 2006

P. R. Nigst, The first modern humans in the Middle Danube Area? New evidence from Willendorf II (Eastern Austria). In: N. J. Conard (Hrsg.), When Neanderthals and Modern $\mathrm{Hu}-$ mans Met. Tübingen 2006, 269-304.

Nigst 2012

P. R. Nigst, The Early Upper Palaeolithic in the Middle Danube Region. Leiden 2012

Nigst 2014

P. R. Nigst, First modern human occupation of Europe: the Middle Danube Region as a case study. In: K. Boyle, R. J. Rabett, C. O. Hunt (Hrsg.), Living in the Landscape. Essays in Honour of Graeme Barker, McDonald Institute for Archaeological Research, Cambridge 2014, 35-47.

Nigst 2019

P. R. Nigst, Approaching the Neanderthal-modern human replacement with population contact scenarios. In: P. C. RAMSL, K. Rebay-Salisbury, P. Trebsche (Hrsg.), Schichtengeschichten. Festschrift für Otto H. Urban, Bonn 2019, 261-279.

Nigst, ANTL-WEISER 2013

P. R. Nigst, W. Antr-Weiser, KG Willendorf, Fundberichte aus Österreich 51/2012, 2013, 242.

Nigst, Haesaerts 2012

P. R. Nigst, P. Haesaerts, L'Aurignacien en Basse Autriche: résultats préliminaires de l'analyse technologique de la couche culturelle 3 de Willendorf II et ses implications pour la chronologie du Paléolithique supérieur ancien en Europe centrale, l'Anthropologie 116, 2012, 575-608.

Nigst et al. 2008a

P. R. Nigst, T. B. Viola, P. Haesaerts, G. Trnka, Willendorf II. In: Venus08 - Art and Lifestyle. Symposium Vienna, 10-14. November 2008. Wissenschaftliche Mitteilungen aus dem Niederösterreichischen Landesmuseum 19, St. Pölten 2008, 31-58.

Nigst et al. 2008b

P. R. Nigst, T. B. Viola, P. Haesaerts, S. Blockley, F. Damblon, C. Frank, M. Fuchs, M. Götzinger, U. Нambach, C. Mallol, L. Moreau, L. Niven, M. Richards, D. Richter, L. Zöller, G. TRnkA, J.-J. Hublin, New research on the Aurignacian of Central Europe: a first note on the 2006 fieldwork at Willendorf II, Quartär 55, 2008, 9-15.

Nigst et al. 2008c

P. R. Nigst, T. B. Viola, P. Haesaerts, S. Blockley, S., F. Damblon, C. Frank, M. Götzinger, U. Нambach, C. Mallol, L. Moreau, L. Niven, D. Richter, M. Richards, L. Zöller, 
J.-J. Hublin, G. Trnka, Neue Forschungen in Willendorf II: Ein Vorbericht über die Grabung 2006, Mitteilungen der Anthropologischen Gesellschaft in Wien 138, 2008, 79-88.

Nigst et al. 2014

P. R. Nigst, P. Haesaerts, F. Damblon, C. Frank-Fellner, C. Mallol, T. B. Viola, M. Götzinger, L. Niven, G. Trnka, J.-J. Hublin, Early modern human settlement of Europe north of the Alps occurred 43,500 years ago in a cold steppe-type environment, Proceedings of the National Academy of Sciences of the U.S.A. 111, 2014, 14394-14399.

Noiret 2004

P. NoIRET, Le Paléolithique supérieur de la Moldavie, l'Anthropologie $108,2004,425-470$.

O'FARRELL 2000

M. O’FARRELl, Les pointes de La Gravette de Corbiac (Dordogne) et considérations sur la chasse au Paléolithique supérieur ancien. In: P. Bodu, C. Constantin (Hrsg.), Approches fonctionnelles en Préhistoire. $\mathrm{XXV}^{\mathrm{e}}$ Congrès Préhistorique de France, Nanterre 24-26 novembre 2000. Paris 2000, 121-138.

Отт 1996

I. Отт, Die Artefakte der jungpaläolithischen Fundstelle von Rosenburg am Kamp, Niederösterreich, Archaeologia Austriaca 80, 1996, 43-114.

Отте 1981

M. Отте, Le Gravettien en Europe Centrale. Dissertationes Archaeologicae Gandenses 20, Brügge 1981.

Отте 1991

M. Отте, Révision de la séquence de Willendorf. In: A. MonTET-White (Hrsg.), Les bassins du Rhin et du Danube au Paléolithique supérieur: environnement, habitat et systèmes d'échange. Études et Recherches Archéologiques de l'Université de Liège 43, Lüttich 1991, 45-60.

Otte, Chirica, Haesaerts 2007

M. Otte, V. Chirica, P. Haesaerts, L'Aurignacien et le Gravettien de Mitoc-Malu Galben (Moldavie Roumaine). Études et Recherches Archéologiques de l'Université de Liège 72, Lüttich 2007.

OTTE et al. 1996

M. Otte, P. Noiret, V. Chirica, I. A. Borziac, Rythme évolutif du Gravettien oriental. In: A. Montet-White, A. Palma di Cesnola, K. Valoch (Hrsg.), The Upper Palaeolithic, Colloquium 12: The Origin of the Gravettian. Actes du $13^{e}$ Congrès international de l'UISPP, Forlì, 8-14 septembre 1996. Série Colloquia 6, Forlì 1996, 213-226.

Pelegrin 2000

J. Pelegrin, Les techniques de débitage laminaire au Tardiglaciaire: critères de diagnose et quelques réflexions. In: B. Valentin, P. Bodu, M. Christensen (Hrsg.), L’Europe centrale et septentrionale au Tardiglaciaire: confrontation des modèles régionaux de peuplement. Actes de la Table-ronde internationale de Nemours, 14-16 mai 1997. Nemours 2000, 73-86.

Peтtitt 2011

P. Pettitt, The Palaeolithic Origins of Human Burial. Oxon 2011.

Peyrony 1933

D. Peyrony, Les industries „aurignaciennes“ dans le bassin de la Vézère, Aurignacien et Périgordien, Bulletin de la Société Préhistorique Française 30, 1933, 543-559.

Peyrony 1937

D. Peyrony, Le Périgordien et l'Aurignacien (Nouvelles observations), Bulletin de la Société Préhistorique Française 33, 1937, 616-619.
Peyrony 1946

D. Peyrony, Une mise au point au sujet de l'Aurignacien et du Périgordien, Bulletin de la Société Préhistorique Française 43, 1946, 232-237.

Pigeot 1987

N. Pigeot, Magdaléniens d'Étiolles: économie de débitage et organisation sociale. Gallia Préhistoire, Supplément 25, Paris 1987.

PitTioni 1932

R. Pittioni, Funde aus Willendorf, Mitteilungen der Anthropologischen Gesellschaft in Wien 62, 1932, 349-360.

P̌̃ICHYSTAL 2013

A. PřIICHYstaL, Lithic Raw Materials in Prehistoric Times of Eastern Central Europe. Brünn 2013.

Pryor et al. 2013

A. J. E. Pryor, M. Steele, M. K. Jones, J. Svoboda, D. G. BeresFORD-Jones, Plant foods in the Upper Palaeolithic at Dolní Vĕstonice? Parenchyma redux, Antiquity 87, 2013, 971-984.

R CORE TEAM 2018

R CORE TEAM, R: A language and environment for statistical computing. R Foundation for Statistical Computing, Vienna, Austria, https://www.R-project.org (letzter Zugriff: 18.9.2019).

Riel-Salvatore, Gravel-Miguel 2013

J. Riel-Salvatore, C. Gravel-Miguel, Upper Palaeolithic mortuary practices in Eurasia: a critical look at the burial record. In: The Oxford Handbook of the Archaeology of Death and Burial. Oxford 2013, 303-346.

Roth, DibBle 1998

B. J. Roth, H. L. Dibble, Production and transport of blanks and tools at the French Middle Paleolithic Site of Combe-Capelle Bas, American Antiquity 63, 1998, 47-62.

ROTTLÄNDER 1975

R. RotTLÄNDER, The formation of patina on flint, Archaeometry 17, 1975, 106-110.

RUTTKAY 1987

E. RuttKay, Ein Brandgrab der Lengyelkultur mit einer Henkelschale aus Ursprung, Niederösterreich, Annalen des Naturhistorischen Museums in Wien 89, 1987, 211-224.

SCHÄFER 1988

D. SCHÄFER, Merkmalanalyse mittelpaläolithischer Steinartefakte. Dissertation, Humboldt-Universität zu Berlin 1988.

SiKora et al. 2017

M. Sikora, A. Seguin-Orlando, V. C. Sousa, A. Albrechtsen, T. Korneliussen, A. KO, S. Rasmussen, I. Dupanloup, P. R. Nigst, M. D. Bosch, G. Renaud, M. E. Allentoft, A. Margaryan, S. V. Vasilyev, E. V. Veselovskaya, S. B. Borutskaya, T. Deviese, D. Comeskey, T. Higham, A. Manica, R. Foley, D. J. Meltzer, R. Nielsen, L. Excoffier, M. Mirazon lahr, L. Orlando, E. Willerslev, Ancient genomes show social and reproductive behavior of early Upper Paleolithic foragers, Science 358/6363, 2017, 659-662.

Simon 1996

U. Simon, Die Schmuckobjekte vom Petersfels in der Sammlung Worm. In: I. Campen, J. Hahn, M. Uerpmann (Hrsg.), Spuren der Jagd - Die Jagd nach Spuren. Festschrift für Hansjürgen Müller-Beck. Tübinger Monographien zur Urgeschichte 11, Tübingen 1996, 203-206.

SOFFER et al. 1998

O. Soffer, J. M. Adovasio, D. C. Hyland, B. Klíma, J. Svoboda, Perishable technologies and the genesis of the Eastern Gravettian, Anthropologie XXXVI/1-2, 1998, 43-68. 
Soriano, Villa, Wadley 2007

S. Soriano, P. Villa, L. Wadley, Blade technology and tool forms in the Middle Stone Age of South Africa: the Howiesons Poort and post-Howiesons Poort at Rose Cottage Cave, Journal of Archaeological Science 34, 2007, 681-703.

SvobOda 1994a

J. Svoвоda, The Pavlov Site, Czech Republic: lithic evidence from the Upper Paleolithic, Journal of Field Archaeology 21, 1994, 69-81. SvOBODA 1994b

J. SvoвоDA, Pavlov I - Excavations 1952-1953. Études et Recherches Archéologiques de l'Université de Liège 66, Dolní Věstonice Studies 2, Lüttich 1994.

SvoBOda 1996

J. Svoвoda, The Pavlovian: typology and behaviour. In: J. SvoвoDA (Hrsg.), Paleolithic in the Middle Danube Region. Spisy Archeologického ústavu Akademie věd ČR v Brně 5, Brünn 1996, 283-301.

SVOBODA 2003

J. SvовоDA, Gravettian and Epigravettian chronologies in the Middle Danube Area. In: F. Widemann, Y. Taborin (Hrsg.), Chronologies géophysiques et archéologiques du Paléolithique supérieur. / Geophysical and Archaeological Chronologies for the Upper Palaeolithic. Proceedings of the International Colloquium of Ravello, $3^{\text {rd }}-8^{\text {th }}$ Mai 1994. Archeologia, storia, cultura 3, Bari 2003, 273-282.

SvOBODA 2007

J. Svoboda, The Gravettian on the Middle Danube, Paléo 19, 2007, 203-220.

SvoBoda 2010

J. Svoвoda, Dolní Věstonice - Pavlov. Mikulov 2010.

Svoboda 2012

J. SvовоDA, Signifikante Gerättypen des Jungpaläolithikums im östlichen Mitteleuropa. In: H. FLoss (Hrsg.), Steinartefakte: Vom Altpaläolithikum bis in die Neuzeit. Tübingen 2012, 481-488.

Svoboda, LožeK, Vlček 1996

J. Svoboda, V. LožEK, E. VLČEK, Hunters between East and West: The Paleolithic of Moravia. New York - London 1996.

Svoboda et al. 2000

J. Svoboda, B. Klíma, L. Jarošová, P. ŠKrdla, The Gravettian in Moravia: climate, behaviour and technological complexity. In: W. Roebroeks, M. Mussi, J. Svoboda, K. Fennema (Hrsg.), Hunters of the Golden Age: The Mid Upper Paleolithic of Eurasia, 30-20.000. Leiden 2000, 197-217.

Svoboda et al. 2011

J. Svoboda, Z. M. Bochennski, V. Čulikova, A. Dohnalova, S. Hladilova, M. Hlozek, I. Horacek, M. Ivanov, M. Kralik, M. Novak, A. J. E. Pryor, S. Sazelova, R. E. Stevens, J. Wilczyński, P. Wojtal, Paleolithic hunting in a southern Moravian landscape: the case of Milovice IV, Czech Republic, Geoarchaeology 26, 2011, 838-866.

SZOMbathy 1909

J. Szombathy, Die Aurignacienschichten im Löß von Willendorf, Korrespondenzblatt der Deutschen Gesellschaft für Anthropologie, Ethnologie und Urgeschichte XL, 1909, 85-88.

Teyssandier 2003

N. Teyssandier, Les débuts de l'Aurignacien en Europe: Discussion a partir des sites de Geissenklösterle, Willendorf II, KremsHundssteig et Bacho-Kiro. Dissertation, Université de Paris $\mathrm{X}$-Nanterre, Paris 2003
Teyssandier 2007

N. Teyssandier, En route vers l'Ouest : Les débuts de l'Aurignacien en Europe. British Archaeological Reports International Series 1638, Oxford 2007.

Thomas, BrandL, Simon 2016

R. Thomas, M. Brande, U. Simon, The Gravettian lithic industry at Krems-Wachtberg (Austria), Quaternary International 406, 2016, 106-119.

TOSTEVIN 2000a

G. Tostevin, Behavioral Change and Regional Variation across the Middle to Upper Paleolithic Transition in Central Europe, Eastern Europe, and the Levant. Dissertation, Harvard University, Cambridge 2000.

Tostevin 2000b

G. Tostevin, The Middle to Upper Paleolithic transition from the Levant to Central Europe: in situ development or diffusion? In: J. Orschiedt, G.-C. Weniger (Hrsg.), Neanderthals and Modern Humans - Discussing the Transition: Central and Eastern Europe from 50.000-30.000 B.P. Wissenschaftliche Schriften des Neanderthal Museums 2, Mettmann 2000, 92-111.

TosTevin 2003a

G. Tostevin, A quest for antecedents: a comparison of the terminal Middle Palaeolithic and early Upper Palaeolithic of the Levant. In: A. N. Goring-Morris, A. Belfer-Cohen (Hrsg.), More Than Meets The Eye: Studies on Upper Palaeolithic Diversity in the Near East. Oxford 2003, 54-310.

Tostevin 2003b

G. Tostevin, Attribute analysis of the lithic technologies of Stránská skála IIIc and IIId in regional and interregional context. In: J. Svoboda, O. Bar-Yosef (Hrsg.), Origins of the Upper Paleolithic in the Brno Basin Moravia, Czech Republic. Cambridge $2003,77-118$.

Tostevin 2011

G. Tostevin, Levels of theory and social practice in the reduction sequence and châine opératoire methods of lithic analysis, PaleoAnthropology, 2011, 351-375.

Tostevin 2012

G. Tostevin, Seeing Lithics: A Middle-Range Theory for Testing for Cultural Transmission in the Pleistocene. Oxford 2012.

Valoch 1981

K. Valoch, Beitrag zur Kenntnis des Pavlovien, Archeologické rozhledy 33, 1981, 279-298.

VERPOORTE 2001

A. Verpoorte, Places of Art, Traces of Fire: A Contextual Approach to Anthropomorphic Figurines in the Pavlovian (Central Europe, 29-24 kyr BP). Archaeological Studies Leiden University 8 , Leiden 2001.

VILLA 1982

P. VILla, Conjoinable pieces and site formation processes, American Antiquity 47, 1982, 276-290.

Villa, Delagnes, Wadley 2005

P. Villa, A. Delagnes, L. Wadley, A late Middle Stone Age artifact assemblage from Sibudu (KwaZulu-Natal): comparisons with the European Middle Paleolithic, Journal of Archaeological Science 32, 2005, 399-422.

WADLEY 2005

L. WADLEY, Putting ochre to the test: replication studies of adhesives that may have been used for hafting tools in the Middle Stone Age, Journal of Human Evolution 49, 2005, 587-601. 
WEINER 1985

J. Weiner, Die Verbesserung der Bearbeitungseigenschaften von amorphen Gesteinsarten durch kontrollierte thermische Behandlung. Mitteilungsblatt der Archaeologica Venatoria 9, Tübingen 1985, 39-47.

WICKHAM 2016

H. Wicкham, ggplot2: Elegant Graphics for Data Analysis. New York 2016.

WiLCZYŃsKi et al. 2015

J. Wilczyński, P. Wojtal, D. Sobieraj, K. Sobczyk, Kraków Spadzista trench C2: new research and interpretations of Gravettian settlement, Quaternary International 359, 2015, 96-113.

WiśNIEWSKi et al. 2015

A. Wiśniewski, T. Peonka, J. Zdzisławl, L. Lenka, A. Traczyk, B. Kufel-Diakowska, J. Raczyk, A. Bajer, The early Gravettian in a marginal area: New evidence from SW Poland, Quaternary International 359, 2015, 131-152.

ZeLINKOVA 2007

M. Zelinkova, Bone and antler industry from Dolni Vestonice I, Acta Musei Moraviae, Scientiae Sociales 92, 2007, 9-51.

Ziehaus 2007

J. Zienaus, Die Silexindustrie der Gravettien-Fundstelle KremsWachtberg, Niederösterreich, Grabung 2005, Archaeologia Austriaca 91, 2007, 7-140.

Viola C. Schmid Institut für Ur-und Frühgeschichte und Archäologie des Mittelalters Eberhard Karls Universität Tübingen Schloß Hobentübingen, Burgsteige 11 72070 Tübingen Deutschland

$\varepsilon$

UMR 7041, ArScAn-AnTET Université Paris Onest Nanterre La Défense 200, avenue de la République 92001 Nanterre Cedex Frankreich viola.schmid@uni-tuebingen.de orcid.org/ 0000-0001-8630-4782

Marjolein D. Bosch McDonald Institute for Archaeological Research University of Cambridge Downing Street Cambridge CB2 3ER Großbritannien dmb75@cam.ac.uk orcid.org/0000-0002-2829-3832
Institut für Orientalische und Europäische Archäologie Österreichische Akademie der Wissenschaften Hollandstraße 11-13 1020 Wien Österreich michael.brandl@oeaw.ac.at orcid.org/0000-0002-0214-8231

Michael Götzinger Institut für Mineralogie und Kristallographie

Universität Wien

Althanstraße 14 1090 Wien Österreich michael.goetzinger@univie.ac.at

Philip R. Nigst Department of Archaeology University of Cambridge

Downing Street Cambridge CB2 3DZ

Großbritannien prn25@cam.ac.uk orcid.org/0000-0001-7330-8768 
